\title{
Simulation of thin film titanium dye sensitized photovoltaic devices
}

\author{
T. Hewitt
}

A thesis submitted to the Faculty of Graduate Studies and Research in partial fulfillment of the requirements for the degree of Masters of Applied Science

Department of Electronics

Carleton University,

Ottawa, Ontario, Canada

May 2008

(c) Thomas Hewitt 


$\begin{array}{ll}\begin{array}{l}\text { Library and } \\ \text { Archives Canada }\end{array} & \begin{array}{l}\text { Bibliothèque et } \\ \text { Archives Canada }\end{array} \\ \begin{array}{l}\text { Published Heritage } \\ \text { Branch }\end{array} & \begin{array}{l}\text { Direction du } \\ \text { Patrimoine de l'édition }\end{array} \\ \begin{array}{l}\text { 395 Wellington Street } \\ \text { Ottawa ON K1A ON4 } \\ \text { Canada }\end{array} & \begin{array}{l}\text { 395, rue Wellington } \\ \text { Ottawa ON K1A 0N4 } \\ \text { Canada }\end{array}\end{array}$

Your file Votre référence ISBN: 978-0-494-44044-5

Our file Notre référence

ISBN: 978-0-494-44044-5

NOTICE:

The author has granted a nonexclusive license allowing Library and Archives Canada to reproduce, publish, archive, preserve, conserve, communicate to the public by telecommunication or on the Internet, loan, distribute and sell theses worldwide, for commercial or noncommercial purposes, in microform, paper, electronic and/or any other formats.

The author retains copyright ownership and moral rights in this thesis. Neither the thesis nor substantial extracts from it may be printed or otherwise reproduced without the author's permission.
AVIS:

L'auteur a accordé une licence non exclusive permettant à la Bibliothèque et Archives Canada de reproduire, publier, archiver, sauvegarder, conserver, transmettre au public par télécommunication ou par l'Internet, prêter, distribuer et vendre des thèses partout dans le monde, à des fins commerciales ou autres, sur support microforme, papier, électronique et/ou autres formats.

L'auteur conserve la propriété du droit d'auteur et des droits moraux qui protège cette thèse. $\mathrm{Ni}$ la thèse ni des extraits substantiels de celle-ci ne doivent être imprimés ou autrement reproduits sans son autorisation.
In compliance with the Canadian

Privacy Act some supporting forms may have been removed from this thesis.

While these forms may be included in the document page count, their removal does not represent any loss of content from the thesis.
Conformément à la loi canadienne sur la protection de la vie privée, quelques formulaires secondaires ont été enlevés de cette thèse.

Bien que ces formulaires aient inclus dans la pagination, il n'y aura aucun contenu manquant.

\section{Canada}




\section{Abstract}

In this work, we explore the possibility of simulating polymer dye sensitized solar cells (DSSC) using semiconductor techniques. A simulation model is designed and built to simulate solar cell structures in one, two and three dimensions, and the model is verified against commercial software Synopsys Medici. Various structures are simulated to find a correlation between structure shape and overall cell efficiency. The results in two dimensions show a reduction in base structure thickness produces higher efficiency. The location of charge generation also effects the efficiency of the cell, but to a lesser degree. Maximum efficiencies of $7.5-10 \%$ were shown in the simulations. Three dimensional simulations were unsuccessful due to computational complexity contributing to excessively long computation times. 


\section{Table of Contents}

Abstract

Acknowledgments

Table of Contents

List of Tables

List of Figures $\quad$ viii

1 Introduction 1

2 Background $\quad 8$

2.1 Basic equations for semiconducting materials . . . . . . . . . . . . 8

2.2 The finite difference method . . . . . . . . . . . . . . . . . 12

2.3 The Newton-Raphson iteration method . . . . . . . . . . . . . 13

3 Simulator design and verification $\quad 16$

3.1 Design methodology . . . . . . . . . . . . . . . . 16

3.1 .1 1D design methodology . . . . . . . . . . . . . . . . . 16

3.1 .2 3D design methodology . . . . . . . . . . . . . . . 20

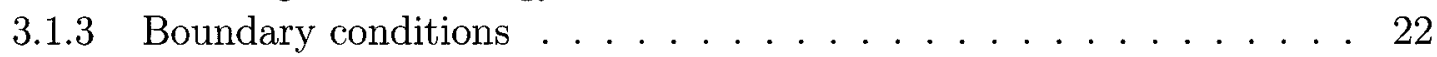

3.1 .4 Initial conditions . . . . . . . . . . . . . . . 23

3.2 Design Verification . . . . . . . . . . . . . . . . . . . . 24

3.2 .1 One dimensional PN junctions . . . . . . . . . . . . . . 26

3.2 .2 Photogeneration in PN junctions . . . . . . . . . . 28

3.2.3 Two dimensional PN junction simulations . . . . . . . . . . . 29

3.2 .4 Discussion . . . . . . . . . . . . . . . . . . . . 32

4 Simulation procedure and parameter implementation 34

4.1 Material parameters . . . . . . . . . . . . . . . . 34

4.1 .1 Titanium dioxide . . . . . . . . . . . . . . . 34

4.1 .2 Indium Tin Oxide . . . . . . . . . . . . . . . . . 36 


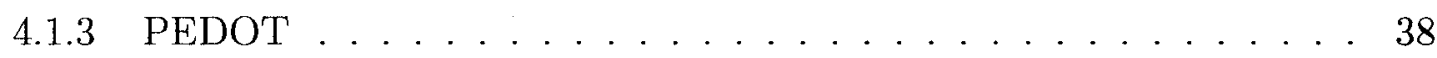

4.1.4 Ruthenium Dye N3 and generation rate modeling . . . . . . . . 40

4.2 Simulation procedure . . . . . . . . . . . . . . 43

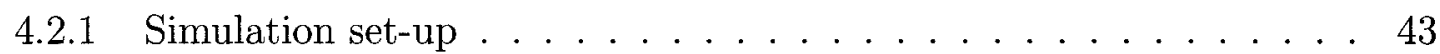

4.2 .2 Simulation flow . . . . . . . . . . . . . . . 46

5 Results 53

5.1 Baseline results . . . . . . . . . . . . . . . . 53

5.2 Two dimensional structuring results . . . . . . . . . . . . . . . . . . . . . . . .

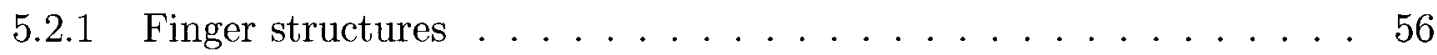

5.2 .2 Complex structuring . . . . . . . . . . . . . 58

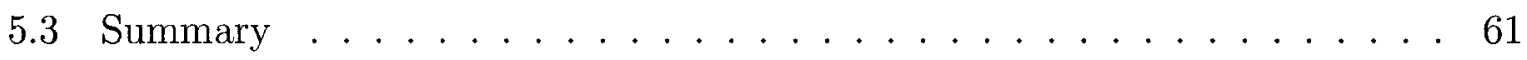

$\begin{array}{lll}6 & \text { Conclusions } & 71\end{array}$

$\begin{array}{ll}\text { References } & \mathbf{7 7}\end{array}$ 


\section{List of Tables}

3.1 Material parameters for silicon . . . . . . . . . . . . . . . . . 26

4.1 Material parameters for rutile titania $\ldots \ldots \ldots \ldots \ldots \ldots$

4.2 Material parameters for ITO . . . . . . . . . . . . . . . 37

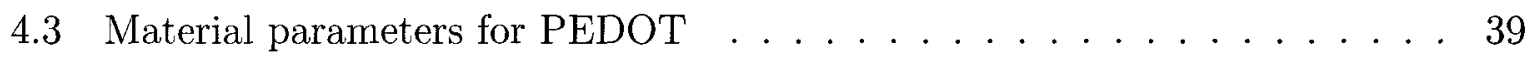

5.1 Maximum efficiency results summary . . . . . . . . . . . . . . 62 


\section{List of Figures}

1.1 Reported timeline of solar cell energy conversion efficiencies (from National Renewable Energy Laboratory (USA)) . . . . . . . . . . . 2

1.2 DSC operation. The HOMO and LUMO state of the dye are denoted by $\mathrm{S} 0$ and $\mathrm{S}^{*}$ respectively. ..................... 3

1.3 Energy band diagram for multilayered polymer DSSC . . . . . . . . . 5

1.4 Microscopic view of nano-structured titania $\ldots \ldots \ldots 6$

2.1 Discretization of boundary value problem . . . . . . . . . . . 12

2.2 Geometric illustration of the Newton-Raphson method . . . . . . . . . 14

3.1 Dividing one dimensional structure $\ldots \ldots \ldots \ldots \ldots$

3.2 Three-dimensional cube discretization and numbering . . . . . . . . . . 21

3.3 Example simulator output for a 1D PN junction with depletion approxi-

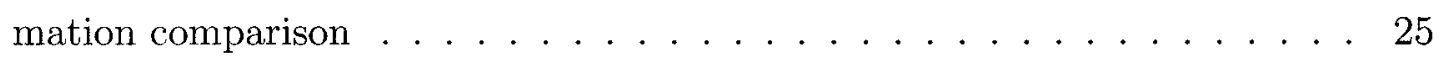

3.4 Carrier concentration results for a 1D PN junction . . . . . . . . 27

3.5 IV curve results for a $1 \mathrm{D}$ PN junction $\ldots \ldots \ldots \ldots \ldots$

3.6 Log IV curve results for a 1D PN junction . . . . . . . . . . . . . . 29

3.7 IV curve results for a $1 \mathrm{D}$ PN junction with photogeneration $\ldots \ldots \ldots 30$ 
3.8 Log IV curve results for a 1D PN junction with photogeneration . . . . . 30

3.9 PVSIM results for a 2D PN junction with $30 \times 5$ unit finger structure . . . . 31

3.10 PVSIM results for a 2D PN junction with 20x20 unit finger structure . . . 31

3.11 PVSIM results for a 2D PN junction with 15x20 unit finger structure . . . 32

4.1 Permittivity and effective electron mass of ITO as a function of carrier

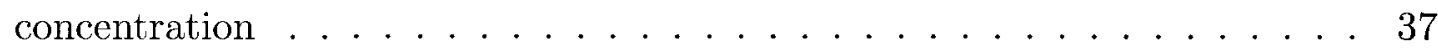

4.2 Hall mobility and resistivity of ITO as a function of carrier concentration . 38

4.3 Optical bandgap of ITO as a function of carrier concentration $\ldots . . . .39$

4.4 Calculated density of states (DOS) of N3 attached to a TiO2 nanocrystal

(a) Total DOS (dashed curve) and N3 PDOS (solid curve). (b) Magnified view of the DOS near the conduction band edge $\ldots \ldots \ldots \ldots 4$

4.5 Absorption spectrum of the N3 Ruthenium dye on $\mathrm{TiO} 2$ substrate . . . . 42

4.6 AM1.5 Spectral irradiance . . . . . . . . . . . . . . . . . . . . . 43

4.7 AM1.5 Photon incidence . . . . . . . . . . . . . . . . . . 44

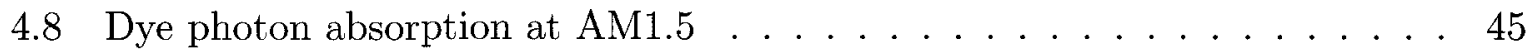

4.9 (a) Structure of titania (b)Generation input . . . . . . . . . . . 46

4.10 Pictorial view of simulation $\ldots \ldots \ldots \ldots \ldots \ldots$

4.11 IV curve for ITO/Titania junction in one dimension . . . . . . . . . . . 48

4.12 Carrier concentration for 1D ITO/Titania junction . . . . . . . . . . 49

4.13 Carrier concentration for 1D Titania/PEDOT junction $\ldots \ldots \ldots \ldots$

4.14 IV curve for 1D ITO/Titania/PEDOT device $\ldots \ldots \ldots \ldots$

4.15 Carrier concentration for 1D ITO/Titania/PEDOT device $\ldots \ldots \ldots . . .51$ 
4.16 Applied voltage IV curve for 1D ITO/Titania/PEDOT device . . . . . . . 51

4.17 Cell efficiency for sample structure . . . . . . . . . . . . . . . 52

5.1 Pictorial view of baseline structures . . . . . . . . . . . . . 54

5.2 IV curve for ITO/Titania/PEDOT device with no structure and varying

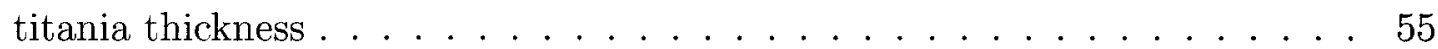

5.3 Efficiency for ITO/Titania/PEDOT device with no structure and varying titania thickness at $g=1 e^{30} \mathrm{~cm}^{-3} \ldots \ldots \ldots \ldots$

5.4 Pictorial view of finger structures . . . . . . . . . . . . 57

5.5 IV curve for ITO/Titania/PEDOT device with finger structure of varying $\operatorname{depth\ldots \ldots \ldots \ldots \ldots \ldots \ldots \ldots \ldots } 58 \ldots \ldots \ldots \ldots \ldots \ldots$

5.6 Efficiency for ITO/Titania/PEDOT device with finger structure of varying thickness at $g=1 e^{29} \mathrm{~cm}^{-3} \ldots \ldots \ldots \ldots \ldots$

5.7 Pictorial view of complex structures . . . . . . . . . . . 60

5.8 Structure shapes (a) (b) (c) (d) and corresponding generation locations (e) (f) $(\mathrm{g})(\mathrm{h}) \ldots \ldots \ldots \ldots \ldots$

5.9 IV curve for ITO/Titania/PEDOT device with complex structures (a)

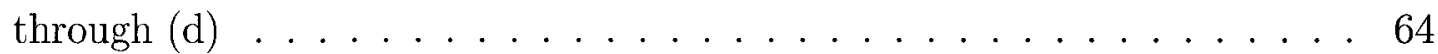

5.10 Efficiency curves for ITO/Titania/PEDOT device with complex structures (a) through $(\mathrm{d}) \ldots \ldots \ldots \ldots \ldots \ldots$

5.11 Output graphs for structure A (a) Log of carrier concentration surface plot (b) Corresponding contour plot (c) Carrier concentration surface plot (d) Voltage surface plot .................... 65 
5.12 Output graphs for structure B (a) Log of carrier concentration surface plot (b) Corresponding contour plot (c) Carrier concentration surface plot (d) Voltage surface plot . . . . . . . . . . . . . 66 66

5.13 Output graphs for structure A (a) Log of carrier concentration surface plot (b) Corresponding contour plot (c) Carrier concentration surface plot (d) Voltage surface plot ..................... 67

5.14 Output graphs for structure A (a) Log of carrier concentration surface plot (b) Corresponding contour plot (c) Carrier concentration surface plot (d) Voltage surface plot . . . . . . . . . . . . . . 68

5.15 Resistance plot for structure D . . . . . . . . . . . . . . . . . 69

5.16 Resistance plot for structure D . . . . . . . . . . . 70 


\section{Chapter 1}

\section{Introduction}

In recent years, growing energy demands and the threat of global warming have brought about much interest in clean energy alternatives. Photovoltaic technology has been among the forefront of these alternatives. A photovoltaic cell, or solar cell, is a device which converts light energy into electrical energy [1]. Unlike fossil fuel burning, nuclear power, and many other energy production methods, photovoltaics have no dangerous by products or environmental impact from energy production.

The photovoltaic effect was first discovered in 1839 by Alexandre Bequerel [2], as he noted a rise in conductance in an electrolyte when exposed to light. The effect was not properly understood until Albert Einstein's work in 1904, for which he was awarded the nobel prize in 1921. It took another 30 years to develop the first production semiconductor solar cell. With a price tag of 1800 dollars US per Watt, and an efficiency of less than 2 percent, these cells were not a viable alternative for energy production.

Figure 1.1 shows the increase of solar cell energy conversion since 1975 [3]. Although not shown in figure 1.1, Spectrolab achieved an efficiency of just over 40 percent in 2006 
with installation cost of less than 3 dollars per Watt [4].

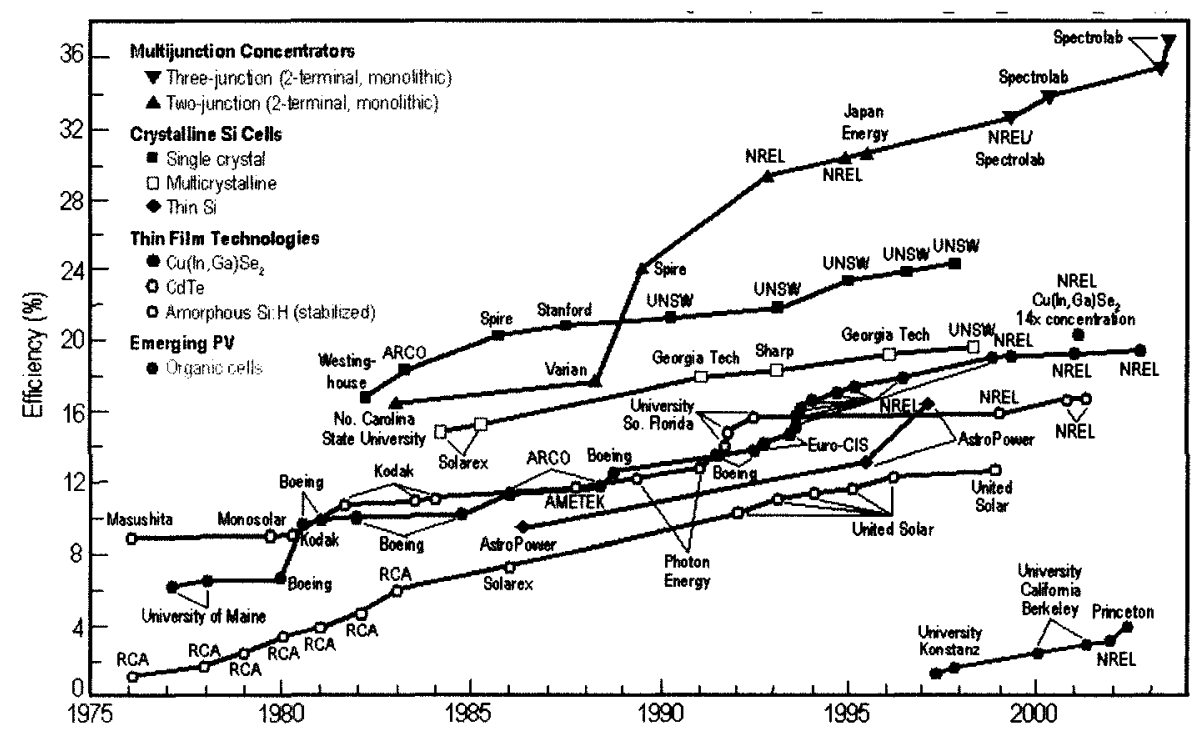

Figure 1.1: Reported timeline of solar cell energy conversion efficiencies (from National Renewable Energy Laboratory (USA))

Bulk silicon solar cells are basically a diode, or PN junction. When exposed to light, a photon can pass through the device or it can generate heat within the cell. If the energy of the photon is greater than the bandgap energy, the photon may excite an electron from the valence band to the conduction band, thus generating an electron hole pair [5]. The generation causes an open circuit voltage across the cell, and when attached to a load, produces power as the charge flows from the device. The power output from the cell is then calculated by multiplying the current achieved by the device multiplied by the operating voltage. By changing the load, the maximum power of the cell can be determined. The efficiency of the cell is the ratio of generated electrical power to the input power of the incoming light [2]. 
Thin film photovoltaic technologies attempt to use less semiconducting material to reduce production costs. The cells tend to have lower conversion efficiency. Recent advances have shown higher efficiencies by using multi-layer devices [2]. The highest efficiencies are produced using a combination of concentrators and multi-layered devices. The concentrators focus the light much like a magnifying glass, and the different layers absorb light from different parts of the spectrum to achieve maximum absorption.

In 1991, M. Gratzel published his results for a dye sensitized solar cell(DSSC) with efficiencies up to 7\% [1]. The DSSC is a very economical alternative to PN junction solar cells, but up to this point had not achieved any reasonable efficiencies. In contrast to semiconductor photocells where the semiconductor produces and transports the charge, the DSSC separates the two functions. Figure 1.2 shows the basic operation of Gratzel's DSSC [6].

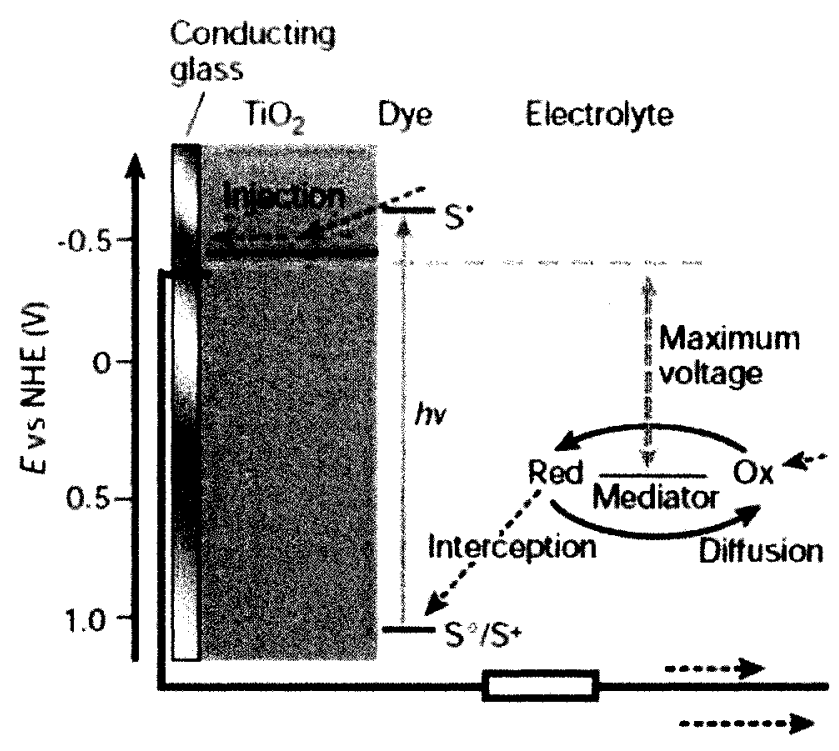

Figure 1.2: DSC operation. The HOMO and LUMO state of the dye are denoted by S0 and $\mathrm{S}^{*}$ respectively. 
There are 5 separate layers in the Gratzel cell, the conducting glass substrate, the titania structure, the dye and the electrolyte and the platinum electrode. Numerous techniques are available to deposit he titania nano-structure on the glass. Attached to the titania is a monolayer of the charge transfer dye, and the whole structure is embedded in electrolyte. The charge separation takes place in the dye due to absorption of photons causing the electron to be excited from the highest occupied molecular orbital (HOMO) to the lowest unoccupied molecular orbital (LUMO) [6]. The charge is injected into the conduction band of the titania and is transported to the conducting glass. The dye sensitizers allow absorption over a large spectral range from UV to near IR. Recent advances in sensitizers and nano-structuring have brought the efficiency of DSSC's up to $10 \%[1]$.

In recent years, researchers have been looking for a solution to produce thin, flexible DSSC solar cells which can be applied to any surface such as office building windows [7-10]. One method is to replace the glass substrate with a plastic substrate and replace the electrolyte with a conducting polymer. The glass can be easily replaced with an indiumtin-oxide (ITO) layered plastic substrate. The electrolyte is replaced with a conducting polymer such as PEDOT [7], lithium salt of poly-thiophene-3-acetic acid (PTAA-Li) [8], or fluorene-bithiophene hole-transporting polymers [10]. Figure 1.3 shows an example band diagram of the cell operation using a conducting fluorene-based polymer F8T2 and PEDOT layer [7].

The charge is separated in the dye, embedded between the titania layer and the polymer. The electron is then injected into the conduction band of the titanium and flows 


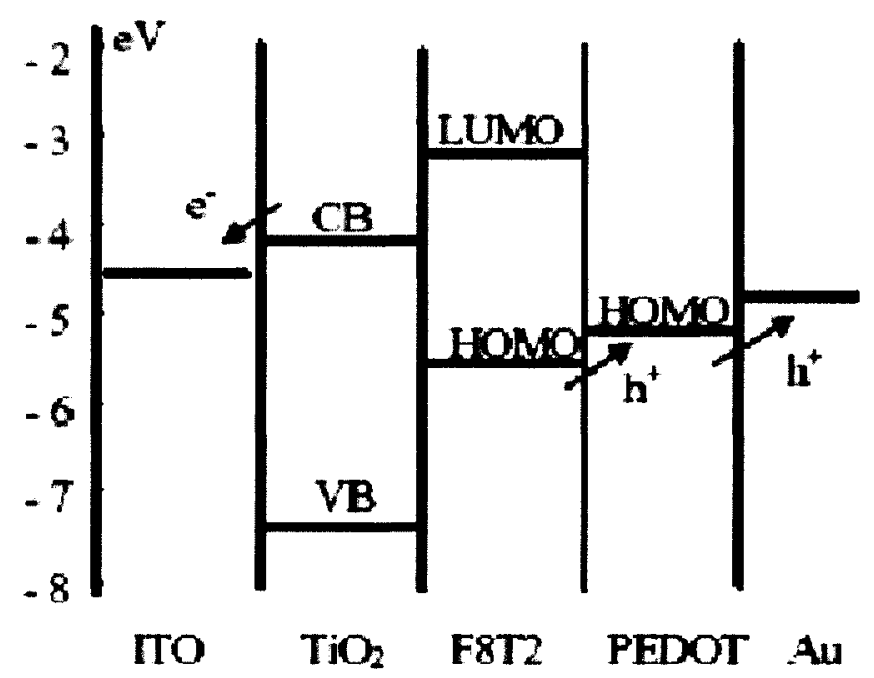

Figure 1.3: Energy band diagram for multilayered polymer DSSC

into the conduction band of the ITO. The hole is conducted through the HUMO levels of the polymers.

The key feature to both Gratzel's DSSC design and to polymer based DSSC's is the structuring of the titania [1,9]. Figure 1.4 shows a sample of a nano-structured film at the microscopic level [11]. The large effective surface area of the nano-structure embeds a large number of dye molecules for light harvesting. The near transparency of the titania itself allows the light to reach the majority of the dye molecules, thus producing a larger amount of charge separation leading to greater efficiencies.

The following work explores the possibility of simulating polymer DSSC's using semiconductor simulation techniques. Being that titania is a very poor conductor, very little is known as to how the charge is transported from the dye to the ITO layer. The titania structure determines the placement of the charge generation, and again very little is 


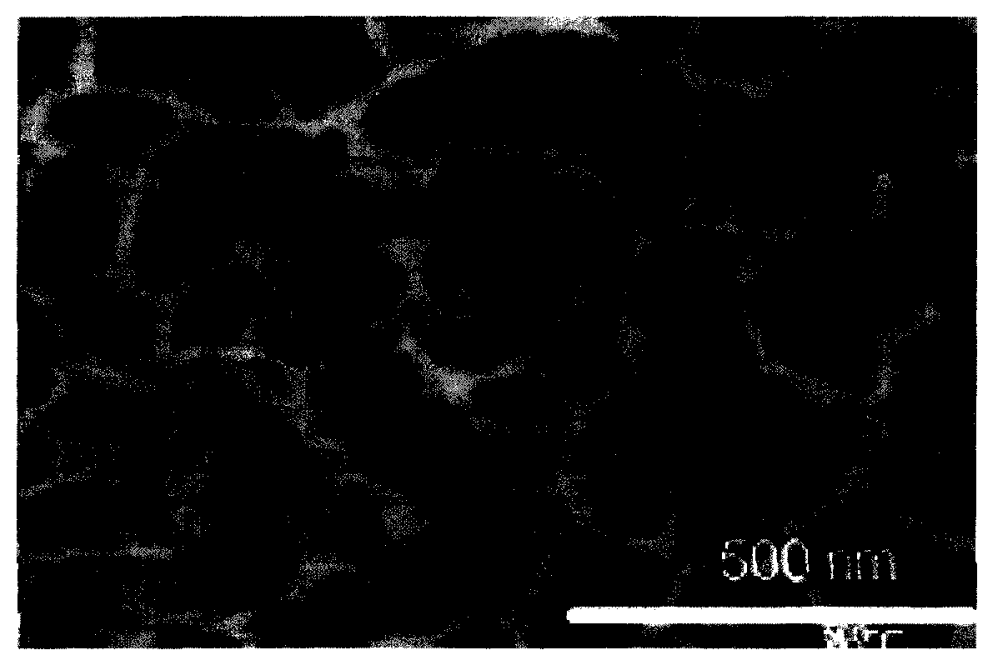

Figure 1.4: Microscopic view of nano-structured titania

known about what effect titania structure has on overall efficiency of the cell.

Initially, it was believed that the problem could be simulated using existing simulation software. The commercial software Medici, produced by Synopsys, claims to be capable of simulating multi-junction devices in two dimensions. It allows the input of complex structures, simulates photo-generation, and is able to incorporate new materials with programable characteristics. Early attempts with this software however exposed a few vital weaknesses. After inputting the desired material parameters for titania, PEDOT, and ITO, the simulator would not converge on solutions to extremely simple single junction problems. After looking deeper into the workings of Medici, it was determined that the initial guess used in the Newton-Raphson algorithm is based on the charge neutrality assumption, and that all other types of guesses are based upon the initial guess or a previous iteration. The charge neutrality assumption produces a guess very close to the solution for materials with similar material parameters and so the solution converges very well for simple silicon junctions or other semiconductor junctions such as GaAs/Ge. 
The materials in the polymer DSSC differ greatly in their material parameters and the initial guess using the charge neutrality assumption is not close enough to the solution to produce convergence.

Another problem arose when dealing with photogeneration. Medici contains an optical device advanced application module to simulate transmission through a device, ray-tracing propagation, and various absorption phenomena. It does not allow a specific user defined input to indicate the exact placement of photogeneration. This is crucial to simulate generation solely in the dye monolayer within the device.

With the many shortcomings of Medici, another alternative had to be found. After looking for any other suitable simulation tool with no success, it was decided that the simulator would be built from the ground up. Using Matlab programmable software, a simulator was designed and built to solve the Poisson and continuity equations using the Newton-Raphson algorithm. The software was designed solely for the purpose of simulating polymer DSSC's and as such is designed to handle complex structures, user defined generation locations, and one, two and three dimensional problems.

The remainder of this work is divided into 5 chapters. Chapter 2 reviews a few background topics needed to understand the simulating techniques discussed. Chapter 3 discusses the design of the simulator used in this work, and shows verification that the simulator functions properly. Chapters 4 and 5 discuss the implementation of a typical simulation and review the results achieved for different titania structures. Finally, chapter 6 provides a summary, conclusions, and suggestions for future work in this area. 


\section{Chapter 2}

\section{Background}

In this chapter we give a brief overview of the underlying mathematical theory involved in the numerical model described within this work. The equations governing electron and hole movement in semiconducting materials is discussed, as well as the Newton-Raphson method for finding a solution to a set of non-linear equations. The finite-difference method for discretizing a finite problem is reviewed.

\subsection{Basic equations for semiconducting materials}

There are several equations governing the hole and electron movement within any semiconducting material. The current-density equations describe the movement of holes or electrons consisting of a drift component and a diffusion component [5].

$$
\begin{aligned}
& J_{n}=q \mu_{n} n \epsilon+q D_{n} \nabla n \\
& J_{p}=q \mu_{p} p \epsilon-q D_{p} \nabla p
\end{aligned}
$$


The $\mathrm{n}$ and $\mathrm{p}$ denote electrons and holes respectively. $\mathrm{J}$ represents the current density in the material. The first term in both equations represents the drift term due to the movement of electrons and holes due to their charge in electric field $\varepsilon$ and mobility $\mu$. The second term describes movement due to diffusion with constant $D$ and gradient $\nabla n$ or $\nabla p$.

The continuity equations derive from the need of balanced movement [5]. In any one region of space, the change of the number of holes or electrons over time is given by the original number, plus any input, minus any output. The continuity equations are given by

$$
\begin{aligned}
& \frac{d n}{d t}=G_{n}+U_{n}+\frac{1}{q} \nabla J_{n} \\
& \frac{d p}{d t}=G_{p}+U_{p}-\frac{1}{q} \nabla J_{p}
\end{aligned}
$$

where $G$ and $U$ represent generation and recombination respectively. The generation term incorporates electron-hole pairs created from solar generation. The recombination term represents the net recombination due to any deviation from equilibrium determined from the mass action law given by [12]

$$
n p=n_{i}^{2}
$$

where $n_{i}$ is the intrinsic density of the material. The quantity can be negative to show net generation. 
By noting that the electric field is the negative gradient of the potential ie $\varepsilon=-\nabla V$, and by combining equations 2.1.1,2.1.2,2.1.3 and 2.1.4, the problem reduces to a series of two equations of three unknowns.

$$
\begin{gathered}
\frac{d n}{d t}=D_{n} \nabla^{2} n-n \mu_{n} \nabla^{2} V+G_{n}+U_{n} \\
\frac{d p}{d t}=D_{p} \nabla^{2} p+p \mu_{p} \nabla^{2} V+G_{p}+U_{p}
\end{gathered}
$$

It is worth noting that the diffusion constant can be expressed in terms of the mobility by [12]:

$$
D_{n}=\frac{k T}{q} \mu_{n}
$$

In order to solve for carrier concentrations $n$ and $p$, and potential $V$, one more equation is required. The Poisson equation relates the potential to the charge in a device by [5]

$$
\nabla^{2} V=-\frac{\rho}{\varepsilon_{r} \varepsilon_{0}}
$$

where $\rho$ denotes charge density and $\epsilon_{r}$ and $\epsilon_{0}$ represent the relative and free space permittivity respectively. In a semiconductor material, the charge can be denoted as [12]

$$
\rho=q\left(n-p+N_{a}-N_{d}\right)
$$

where $N_{a}$ and $N_{d}$ are the acceptor and donor doping concentrations. Equation 2.1.9 then becomes 


$$
\nabla^{2} V=-\frac{-q}{\epsilon_{r} \epsilon_{0}}\left(n-p+N_{a}-N_{d}\right)
$$

To determine the efficiency of a photovoltaic cell, the steady state IV curve is required. Under steady state conditions, the rate of change of both holes and electrons over time can be set to zero. The problem is now a set of three equations in three unknowns.

$$
\begin{aligned}
& D_{n} \nabla^{2} n-n \mu_{n} \nabla^{2} V+G_{n}+U_{n}=0 \\
& D_{p} \nabla^{2} p+p \mu_{p} \nabla^{2} V+G_{p}+U_{p}=0 \\
& \nabla^{2} V=-\frac{q}{\varepsilon_{r} \varepsilon_{0}}\left(n-p+N_{a}-N_{d}\right)
\end{aligned}
$$

Equations 2.1.12 to 2.1.14 are mostly linear, except for the non-linear terms coming from the drift term. Non-linearities can also be present in the recombination and generation terms. The problem now consists of solving the set of three coupled non-linear differential equations.

The equations can be arranged in matrix form with non-linear vector $f(x)$, linear matrix $\bar{A}$ and constants vector $\vec{b}$.

$$
\begin{gathered}
\bar{A} \vec{x}+f(\bar{x})+b=0 \\
{\left[\begin{array}{ccc}
D_{n} \nabla^{2} & 0 & 0 \\
0 & D_{n} \nabla^{2} & 0 \\
1 & -1 & \frac{\varepsilon_{r} \varepsilon_{o}}{q} \nabla^{2}
\end{array}\right]\left[\begin{array}{c}
n \\
p \\
V
\end{array}\right]+\left[\begin{array}{c}
-n \mu_{n} \nabla^{2} V \\
p \mu_{p} \nabla^{2} V \\
0
\end{array}\right]+\left[\begin{array}{c}
G_{n}+U_{n} \\
G_{p}+U_{p} \\
N_{A}-N_{D}
\end{array}\right]=0}
\end{gathered}
$$




\subsection{The finite difference method}

The finite difference method is a method of approximating the derivatives of a function [13]. It comes in very useful when attempting to find numerical solutions to differential problems. There are three common types of finite difference expressions. Forward difference is expressed by $\triangle f(x)=f(x+h)-f(x)$, backwards difference is expressed by $\nabla f(x)=f(x)-f(x-h)$, and central difference $\delta f(x)=f(x+0.5 h)-f(x-0.5 h)$. The derivative of a function is defined as [14]:

$$
f^{\prime}(x)=\lim _{h \rightarrow 0} \frac{f(x+h)-f(x)}{h}=\frac{\triangle f(x)}{h}
$$

As $h$ approaches a very small value, the forward difference divided by $h$ approximates the derivative of $f(x)$.

When dealing with boundary value problems, the problem is first divided into $\mathrm{N}$ discrete parts between all boundaries. If dealing with a 1 dimensional problem, the problem is divided between 0 and length $L$ in $N$ parts of size $h=L / N$. Figure 2.1 shows a illustrative view of discretizing a $1 \mathrm{D}$ problem.

\begin{tabular}{l|l|l|l|}
\multicolumn{4}{c}{$+n-1$} \\
\hline
\end{tabular}

Figure 2.1: Discretization of boundary value problem

In one dimension, the derivative of the function can be written using the the forward difference method as shown in equation 2.2.2. A very small derivation using the central difference method gives the second derivative in equation 2.2.3. 


$$
\begin{gathered}
f^{\prime}(x)=\frac{f\left(x_{i+1}\right)-f\left(x_{i}\right)}{h} \\
f^{\prime \prime}(x)=\frac{\delta}{h} \frac{f\left(x_{i+0.5}\right)-f\left(x_{i-0.5}\right)}{h}=\frac{f\left(x_{i+1}\right)-2 f\left(x_{i}\right)+f\left(x_{i-1}\right)}{h^{2}}
\end{gathered}
$$

With similar logic to equation 2.2.3, the first and second derivatives in three dimensions are given by [14]:

$$
\begin{array}{r}
\nabla f(x, y, z)=\frac{1}{h} f\left(x_{i+1}, y_{i}, z_{i}\right)+f\left(x_{i}, y_{i+1}, z_{i}\right)+f\left(x_{i}, y_{i}, z_{i+1}\right)-3 f\left(x_{i}, y_{i}, z_{i}\right) \\
\nabla^{2} f(x, y, z)=\frac{1}{h^{2}} f\left(x_{i+1}, y_{i}, z_{i}\right)+f\left(x_{i}, y_{i+1}, z_{i}\right)+f\left(x_{i}, y_{i}, z_{i+1}\right)+f\left(x_{i-1}, y_{i}, z_{i}\right)+ \\
f\left(x_{i}, y_{i-1}, z_{i}\right)+f\left(x_{i}, y_{i}, z_{i-1}\right)-6 f\left(x_{i}, y_{i}, z_{i}\right)
\end{array}
$$

The finite difference definitions shown for one dimension in equation 2.2.2 to 2.2.3 and for three dimensions in 2.2.4 to 2.2.5 can be substituted into equation 2.1.16 to form a set of coupled non-linear algebraic equations in matrix format.

\subsection{The Newton-Raphson iteration method}

It was decided to solve equation 2.1.15 using the Newton-Raphson iteration method [15]. A graphical view of the NR method is shown in figure 2.2.

The NR method begins with an initial guess for the solution $x_{i}$ and determines the value of the function at this point $f\left(x_{i}\right)$ and the value of the derivative of the function 


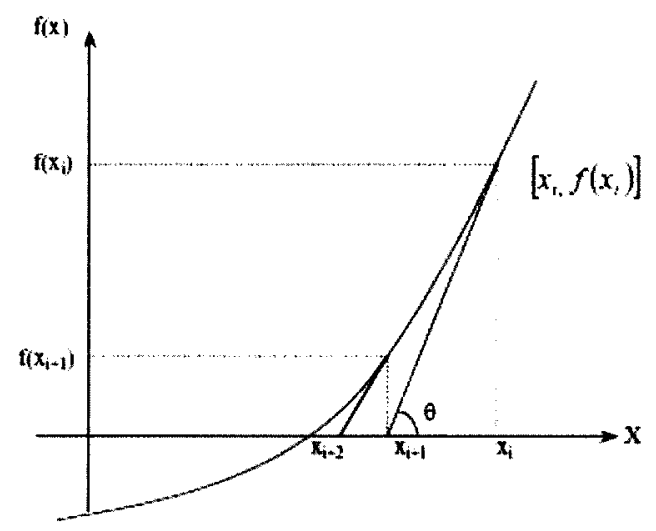

Figure 2.2: Geometric illustration of the Newton-Raphson method

$f^{\prime}\left(x_{i}\right)$. The next guess for the root $x_{i+1}$ is the point where the tangent intersects the horizontal axis. In mathematical terms [15];

$$
x_{i+1}=x_{i}-\frac{f\left(x_{i}\right)}{f^{\prime}\left(x_{i}\right)}
$$

The method continues to iterate until the solution is sufficiently close to the root within desired error tolerance. The NR method is very good at converging to the root when it is very close to the solution, however, convergence is by no means guaranteed for any initial guess. The choice of initial guess is therefore extremely important when using the NR method.

The NR method can also be used to solve a system of equations. Let the system equations for this work be;

$$
f_{k}(\vec{x})=\bar{A} \vec{x}+f_{x}(\vec{x})-\vec{b}
$$


The linear terms appear in matrix $A$, the non-linear terms in function $f_{x}$ and the constants in vector $b$. The derivative of 2.1 .10 with respect to $x_{k}$ is then

$$
f_{k}^{\prime}(\vec{x})=\bar{A}+\bar{J}(\vec{x})
$$

where $J$ is the Jacobian matrix of function $f_{x}$. The NR algorithm becomes:

$$
\overrightarrow{x_{i+1}}=\overrightarrow{x_{i}}-\left(\vec{A}+\bar{J}\left(\overrightarrow{x_{i}}\right)\right) \backslash\left(\vec{A} \overrightarrow{x_{i}}+f_{x}\left(\overrightarrow{x_{i}}\right)-\vec{b}\right)
$$

The $\bar{A}$ matrix and the $\vec{b}$ vector are constant for a given problem, and $\overrightarrow{x_{i}}$ is already known. The Jacobian and the non-linear functions need to be calculated at every iteration. A large gaussian elimination takes place to calculate $\overrightarrow{x_{i+1}}$, making the method computationally expensive. 


\section{Chapter 3}

\section{Simulator design and verification}

The following chapter describes the design of the model used to simulate photovoltaic devices. An overview of how the model is derived from basic equations, and a verification of the model versus commercially available software is included.

\subsection{Design methodology}

The simulation model is designed to solve one, two and three dimensional problems. The model uses a one dimensional analysis module and a three dimensional module that can be simplified to two dimensions if needed. The one dimensional module will be discussed first as it is easier to follow the methodology and visualize the devices.

\subsubsection{D design methodology}

The problem to be solved is a one dimensional semiconductor structure from $x=0$ to $x=L$, where $L$ is the length of the structure. The device can contain any number of different materials with their own material parameters for mobility, permittivity, diffusion coefficient, doping, and intrinsic density. The generation rate can also vary over the 
structure.

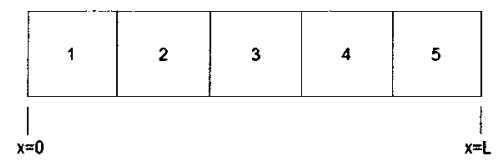

Figure 3.1: Dividing one dimensional structure

The first step is to discretize the structure along the $\mathrm{x}$-axis, numbering the individual node from low to high, each part with size $h$. Figure 3.1 shows a visual diagram of the labeling and discretization. The finite difference method from equations 2.2.2 and 2.2.3 can then be used and combined with equations 2.1.12 to 2.1.14 to form three equations for every individual node $i$.

$$
\begin{gathered}
\frac{\epsilon_{r} \epsilon_{0}}{h^{2}}\left(V_{i-1}-2 V_{i}+V_{i+1}\right)=q\left(n-p+N_{A}-N_{D}\right) \\
\frac{1}{h^{2}}\left(D_{n(i-1)} n_{i-1}-2 D_{n(i)} n_{i}+D_{n(i+1)} n_{i+1}\right)+\frac{1}{h^{2}}\left(n_{i} u_{n(i)}\left(-V_{i+1}+V_{i}\right)-n_{i-1} u_{n(i-1)}\left(-V_{i}+V_{i-1}\right)\right)=0 \\
\frac{1}{h^{2}}\left(D_{p(i-1)} p_{i-1}-2 D_{p(i)} p_{i}+D_{p(i+1)} p_{i+1}\right)+\frac{1}{h^{2}}\left(-p_{i} u_{p(i)}\left(-V_{i+1}+V_{i}\right)+p_{i-1} u_{p(i-1)}\left(-V_{i}+V_{i-1}\right)\right)=0
\end{gathered}
$$

The equations are arranged in matrix format, with equation $\vec{A} \vec{x}+f_{x}(\vec{x})-\vec{b}$ and Jacobian $J$. The model attempts to solve for voltage, and hole/electron densities simultaneously. This causes large singularities in the matrices due to scaling issues between voltage values of order $10^{0}$ and carrier densities of order $10^{16}$. Two scaling factors $s_{1}$ and $s_{2}$ are introduced, and the solution is solved for $\hat{V}$ where $\hat{V}_{i}=s_{1} V_{i} \epsilon_{r(i)}$. The local matrices and vectors are shown in equation 3.1.4. 
$\bar{A}=\left[\begin{array}{ccccccccc}\frac{s_{2} \varepsilon_{o}}{s_{1} q h^{2}} & -\frac{2 s_{2} \varepsilon_{o}}{s_{1} q h^{2}} & \frac{s_{2} \varepsilon_{o}}{s_{1} q h^{2}} & 0 & -s_{2} & 0 & 0 & +s_{2} & 0 \\ 0 & 0 & 0 & \frac{D_{n(i-1)}}{h^{2}} & \frac{-2 D_{n(i)}}{h^{2}} & \frac{D_{n(i+1)}}{h^{2}} & 0 & 0 & 0 \\ 0 & 0 & 0 & 0 & 0 & 0 & \frac{D_{p(i-1)}}{h^{2}} & \frac{D_{p(i)}}{h^{2}} & \frac{D_{p(i+1)}}{h^{2}}\end{array}\right]$

$\vec{x}=\left[\begin{array}{c}\hat{V}_{i-1} \\ \hat{V}_{i} \\ \hat{V}_{i+1} \\ n_{i-1} \\ n_{i} \\ n_{i+1} \\ p_{i-1} \\ p_{i} \\ p_{i+1}\end{array}\right]$

$\vec{f} x=\left[\begin{array}{c}0 \\ \frac{1}{s_{1} h^{2}}\left[n_{i} u_{n i}\left(-\hat{V}_{i+1} / \varepsilon_{r(i+1)}+\hat{V}_{i} / \varepsilon_{r(i)}\right)-n_{i-1} u_{n(i-1)}\left(-\hat{V}_{i} / \varepsilon_{r(i)}+\hat{V}_{i-1} / \varepsilon_{r(i-1)}\right)\right) \\ \frac{1}{s_{1} h^{2}}\left[-p_{i} u_{p i}\left(-\hat{V}_{i+1} / \varepsilon_{r(i+1)}+\hat{V}_{i} / \varepsilon_{r(i)}\right)+p_{i-1} u_{p(i-1)}\left(-\hat{V}_{i} / \varepsilon_{r(i)}+\hat{V}_{i-1} / \varepsilon_{r(i-1)}\right)\right.\end{array}\right]$

$\vec{b}=\left[\begin{array}{c}s_{2}\left(N_{A}-N_{D}\right) \\ -G_{i} \\ -G_{i}\end{array}\right]$ 


$$
\begin{aligned}
& \bar{J}=\left[\begin{array}{cccc}
0 & 0 & 0 & 0 \\
-\frac{n_{i-1} u_{n(i-1)}}{s_{1} h^{2} \varepsilon_{r(i-1)}} & \frac{n_{i} u_{n i}+n_{i-1} u_{n(i-1)}}{s_{1} h^{2} \varepsilon_{r(i)}} & -\frac{n_{i} u_{n i}}{s_{1} h^{2} \varepsilon_{r(i+1)}} & -\frac{u_{n(i-1)}\left[\frac{-\hat{V}_{i}}{\varepsilon_{r(i)}}+\frac{\hat{V}_{i-1}}{\varepsilon_{r(i-1)}}\right]}{s_{1} h^{2}} \\
\frac{p_{i-1} u_{p(i-1)}}{s_{1} h^{2} \varepsilon_{r(i-1)}} & -\frac{p_{i-1} u_{p(i-1)}+p_{i} u_{p i}}{s_{1} h^{2} \varepsilon_{r(i)}} & \frac{p_{i} u_{p i}}{s_{1} h^{2} \varepsilon_{r(i+1)}} & 0
\end{array}\right. \\
& \left.\begin{array}{cccc}
0 & 0 & 0 & 0 \\
\frac{u_{n i}\left[\frac{-\hat{V}_{i+1}}{\varepsilon_{r(i+1)}}+\frac{\hat{V}_{i}}{\varepsilon_{r(i)}}\right]}{s_{1} h^{2}} & 0 & 0 & 0 \\
0 & \frac{u_{p(i-1)}\left[\frac{-\hat{V}_{i}}{\varepsilon_{r(i)}}+\frac{\hat{V}_{i-1}}{\varepsilon_{r(i-1)}}\right]}{s_{1} h^{2}} & -\frac{u_{p i}\left[\frac{-\hat{V}_{i+1}}{\varepsilon_{r(i+1)}}+\frac{\hat{V}_{i}}{\varepsilon_{r(i)}}\right]}{s_{1} h^{2}} & 0
\end{array}\right]
\end{aligned}
$$

After the local matrices and vectors are formed, they must be assembled in global matrices/arrays. Since there are 3 equations for each node, the global matrices are of size $3 N X 3 N$ and the arrays size $3 N X 1$. The global matrix is of size $12 X 12$ since there are 4 nodes in total. The local matrix is of size 3X9. The row indices are calculated as shown on the left hand side to determine the placement in the global matrix. The placement in the columns is determined by the index of the variable in $\vec{x}$. The quantities from the local matrices are added to existing values in the global matrices to model contributions from all nodes. For example, row 2, for local matrix for node 3 corresponds to the row $n+3=7$ of the global matrix, and column 1 corresponds to $\bar{V}_{2}$ and so goes in column 2 of the global matrix. In this way, all local matrix and vector elements can be mapped into the global matrices/arrays. 


$$
\operatorname{row}(1 x 4+3)\left[\begin{array}{cccccccccc}
1 & 2 & 3 & 4 & 5 & 6 & 7 & 8 & 9 \\
10 & 11 & 12 & 13 & 14 & 15 & 16 & 17 & 18 \\
19 & 20 & 21 & 22 & 23 & 24 & 25 & 26 & 27
\end{array}\right]\left[\begin{array}{c}
\hat{V}_{2} \\
\hat{V}_{3} \\
\hat{V}_{4} \\
n_{2} \\
n_{3} \\
n_{4} \\
p_{2} \\
p_{3} \\
p_{4}
\end{array}\right]
$$

Boundary condition application will be discussed later in this section.

\subsubsection{D design methodology}

The design of a three dimensional simulator is very similar to the design of a 1D simulator as discussed in section 3.1.1. The equations are formed by combining equations 2.2 .4 and 2.2.5 with equations 2.1.12 to 2.1.14. The matrices and arrays from the equations are 
large and complex, and can be found in appendix A.

The three dimensional discretization takes place in the same fashion as the one dimensional case, except the 3D structure is broken into 3 dimensional cubes. Each cube is given an individual number. In this way, the three-dimensional structure is mapped onto one dimensional arrays and matrices. The solution is determined exactly the same way as a one-dimensional problem. A pictorial view of the numbering is shown in figure 3.2.

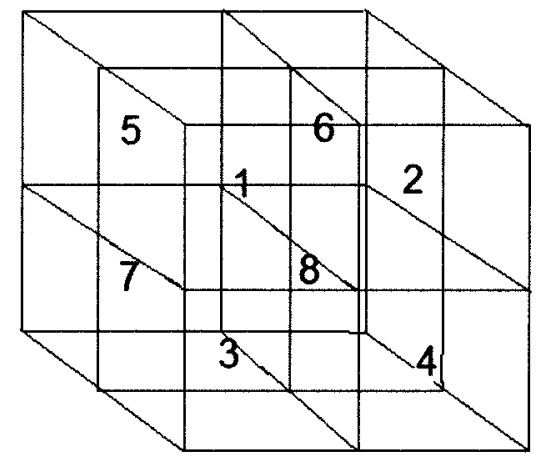

Figure 3.2: Three-dimensional cube discretization and numbering

The node numbering corresponds to equation 3.1.5. The equation maps the three dimensional location of the node with location $x, y, z$ to an individual number $i$. The index $i$ is then used to assemble the local and global matrices. $L Y, L Z$ and $L X$ correspond to the maximum $\mathrm{x}, \mathrm{y}$ and $\mathrm{z}$ values for the structure.

$$
i=L X * L Y *(z-1)+L X *(y-1)+x
$$

By setting $L Z=0$, the problem reduces to a two dimensional problem. In this way, the simulator is used to simulate two dimensional structures. 


\subsubsection{Boundary conditions}

In order to find a solution to equation 2.1 .14 it is necessary to set boundary conditions. There are several types of boundary conditions which are common when solving partial differential equations. The two types of boundary conditions that are used in this work are Neumann and Dirichlet boundary conditions [16].

Dirichlet boundary conditions specify a specific value for the solution at the boundary points [16]. In equation 2.1.14, the values of $n, p$ and $V$ must be specified at the boundaries in order to solve the problem.

Neumann boundary conditions specify the first derivative of the solution at the boundary [16]. In some cases, it is necessary to solve for the electric field value at the boundary. The electric field is simply the derivative of the voltage, and so can be imposed on the boundary using Neumann boundary conditions.

In the case of a one-dimensional problem, the only boundaries exist at $x=1$ and at $x=L X$. The two boundaries correspond to indices $i=1$ and $i=N$ where $\mathrm{N}$ is the number of nodes. To impose Dirichlet boundary conditions, the voltage at node 1 is set to zero, and the voltage at node $\mathrm{N}$ set to a fixed voltage. The values of $\mathrm{n}$ and $\mathrm{p}$ are set to their equilibrium values. To determine the equilibrium values, the mass-action law (equation 2.1.5) is combined with equation 2.1.10. The total charge is assumed to be zero since the boundaries should be far away from the depletion region.

$$
\begin{aligned}
& p^{2}+\left(N_{D}-N_{A}\right) p-n_{i}^{2}=0 \\
& n^{2}-\left(N_{D}-N_{A}\right) n-n_{i}^{2}=0
\end{aligned}
$$


Equations 3.1.6 and 3.1.7 are solved with a root finding algorithm to determine equilibrium values for $\mathrm{n}$ and $\mathrm{p}$.

To impose a zero current situation on a boundary, Neumann boundary conditions are used. To set the electric field to zero at node $i$, the voltage at node $i$ is set to equal the voltage at node i-1. With a zero electric field, there can be no drift across the boundary. To cancel diffusion as well, the $\mathrm{n}$ and $\mathrm{p}$ values at node $\mathrm{i}$ are also set to the values of node i-1.

Two and three dimensional cases are very similar to the one dimensional problem. The boundary conditions at $x=1$ and at $x=L X$ must be set with either Neumann or Dirichlet boundary conditions, but now all elements corresponding to these values of $\mathrm{x}$ must be set instead of a single value. There now exists boundary conditions in the y and $\mathrm{z}$ dimensions as well which must be accounted for. For all the simulations in this work, a repeating structure is wanted, and so periodic boundary conditions are used.

In order to implement periodic boundary conditions, the indices in equation 3.1.5 are modified for any values corresponding to $y=1, z=1, y=L Y, z=L Z$. When dealing with a value corresponding to $y=1$, the indices for $y-1$ do not exist, and are replaced with $L Y$. The values for $y+1$ at $y=L Y$ are replaced with $y=1$. Similar replacements are done with z.

\subsubsection{Initial conditions}

The Newton-Raphson algorithm requires an initial guess for the iterations to begin [15]. The initial guess is very important, as if it is too far from the solution at any given point, 
the algorithm will not converge.

The model in this work does not include modeling of the metal contacts on either end of the device. The built in potential of the junction is therefore incorporated into the applied potential of the device.

The initial guess requires guesses for the electron and hole concentrations as well as the voltage at each point in the device. For a very well know semiconductor junction, such as a regular $\mathrm{P}-\mathrm{N}$ silicon junction, the initial guess can be determined by the depletion approximation [12] for the solution at the built in voltage. The values determined for hole and electron concentrations from the depletion approximation can be combined with the known doping concentrations to determine the charge in the device. From the known charge, the voltage along the device can be solved by solving Poisson's equation (equation 2.1.14).

For an unknown device with a complex structure, determining an approximate solution to the structure at the built in potential is more difficult. The simulator uses a voltage ramping function, solving first for the solution at zero potential across the device, then ramping up the voltage until the current in the device drops to zero. The initial guess uses the equilibrium values found in equations 3.1.6 and 3.1.7, and a zero potential across the device.

\subsection{Design Verification}

The simulator in this work is designed to model photovoltaic devices and so it will be referred to as PVSIM (PhotoVoltaic SIMulator) from this point forward. The simulator 
is design to provide results for electron density, hole density, and voltage across any given device at a given bias and generation rate. The results can be viewed in many different forms. The simulator offers different output graphs, all versus distance. The outputs that can be viewed are hole concentration, electron concentration, voltage, electric field magnitude, flux density, and hole/electron variance. Figure 3.3 shows an example output.
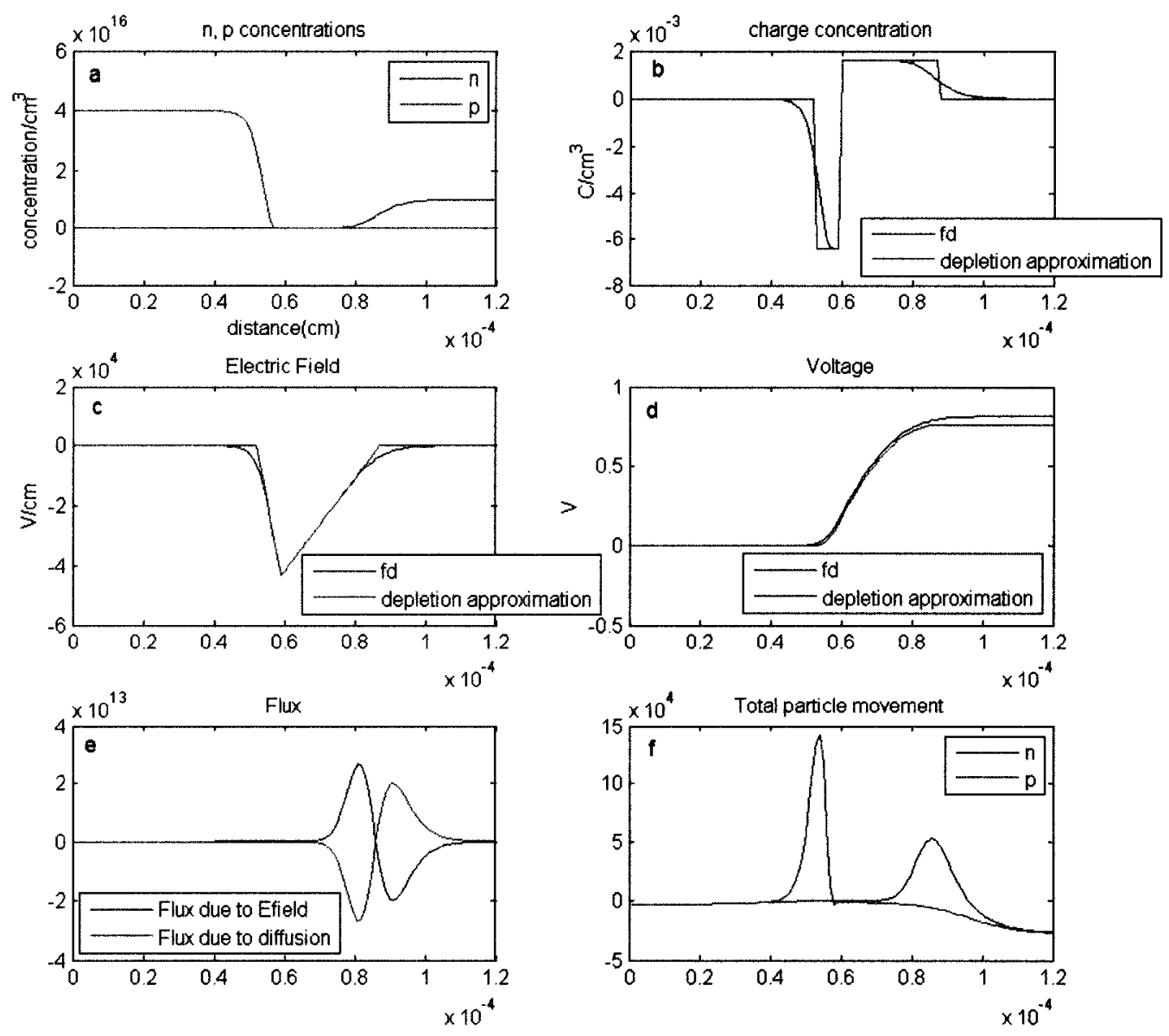


\begin{tabular}{|c|c|}
\hline Material Property & Value \\
\hline$\epsilon_{r}$ & 11.8 \\
\hline$U_{n}$ & $1000 \mathrm{~cm}^{2} / V s$ \\
\hline$U_{p}$ & $500 \mathrm{~cm}^{2} / V s$ \\
\hline$D_{n}$ & $25.85 \mathrm{~cm}^{2} / \mathrm{s}$ \\
\hline$D_{p}$ & $12.92 \mathrm{~cm}^{2} / \mathrm{s}$ \\
\hline$n_{i}$ & $1.45^{10} \mathrm{~cm}^{-3}$ \\
\hline
\end{tabular}

Table 3.1: Material parameters for silicon

to (d) are self explanatory. Graphs (e) and (f) show a few diagnostic tools that can be viewed to help the user determine if there are any problems with the simulation. Graph (e) is the flux density due to diffusion, and to drift. The diffusion and drift outputs should be reverse matched in a steady state solution. Graph (f) is an output of the total variance in hole and electrons from one iteration to another. The error is determined by dividing the variance by the total hole and electron concentrations and determining the maximum value. In some cases, the error calculated is not representative of the actual error due to small carrier densities in some locations in the device. In these situations, it is helpful to look at the variance graph.

To verify the proper functioning of the PVSIM, several simulations are performed with both PVSIM, and the commercial simulator MEDICI. The results are compared in sections 3.2.1 to 3.2.4.

\subsubsection{One dimensional PN junctions}

One of the most studied semiconductor junctions is the simply P-N silicon junction. The material parameters for silicon are very well known and can be found in most solid state physics texts [12]. Table 3.1 shows the material parameters used by MEDICI [17]. 
The values in table 3.1 are used in PVSIM to match MEDICI results. A simulation is performed for a $2 u m$ silicon junction with the junction at $1 \mathrm{um}$. The junction is discretized in 200 equal parts, and the doping is $N_{d}=1 e^{16}$ in the $\mathrm{n}$ region and $N_{a}=4 e^{16}$ in the p region. The Newton-Raphson algorithm iterates until the error is below $1 e^{-4}$. The bias across the junction is modified and the results displayed in terms of charge carrier concentration. The results are shown in figure 3.4.

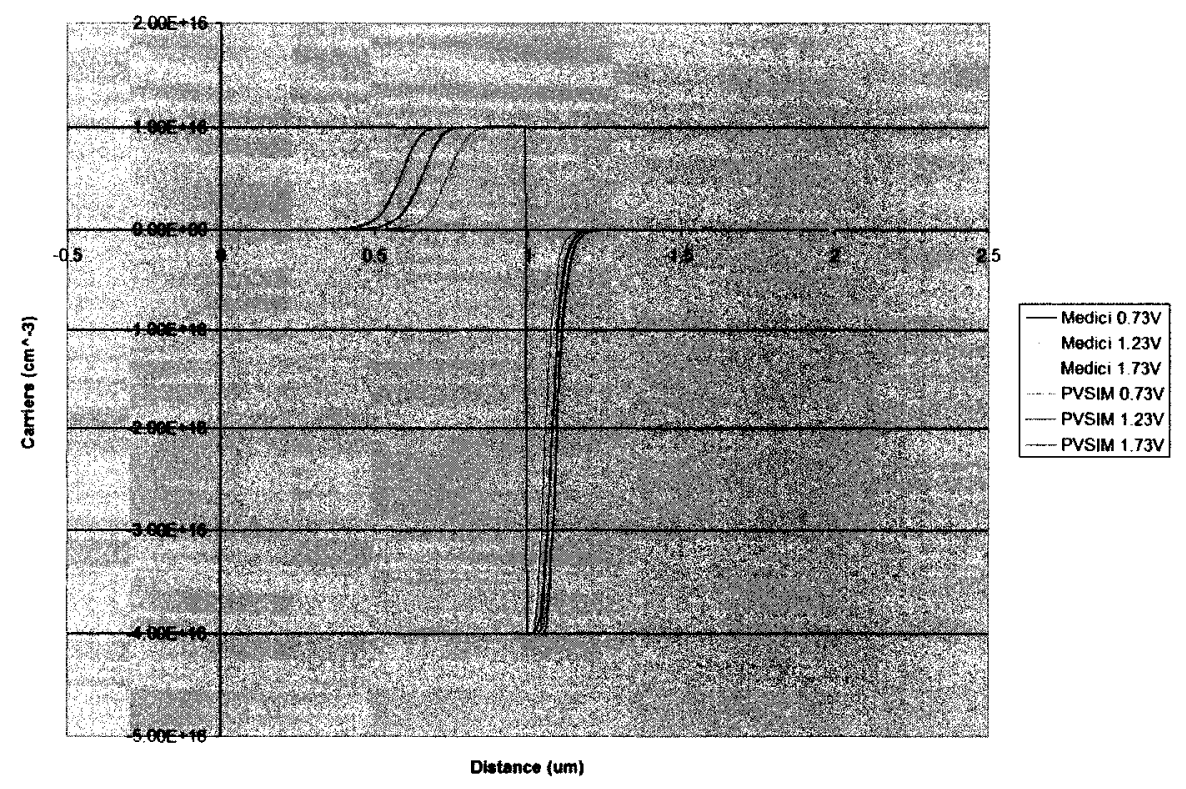

Figure 3.4: Carrier concentration results for a 1D PN junction

The results from MEDICI match those of PVSIM for charge carrier concentration very closely. The junction is simulated in both MEDICI and PVSIM for a range of fixed voltages and the current is measured across the device. The IV curve is shown in figure 3.5, and a $\log$ plot shown in figure 3.6.

The results for the IV curve show a very close match between both results. There is a slight discrepancy at low bias voltage. The discrepancy is believed to be due to numerical 


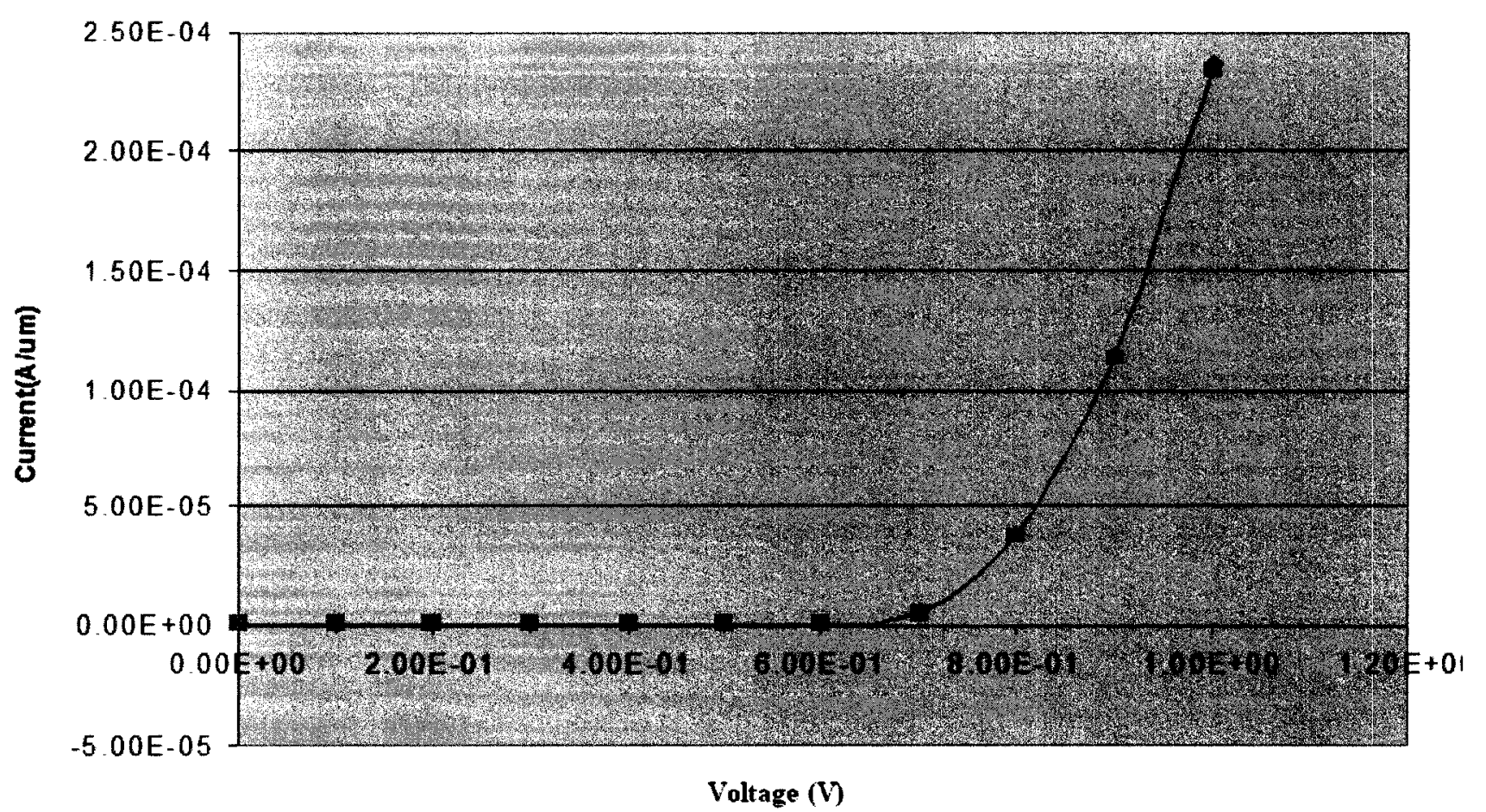

Figure 3.5: IV curve results for a 1D PN junction

considerations.

\subsubsection{Photogeneration in $\mathrm{PN}$ junctions}

To test the photogeneration function of PVSIM, an identical simulation to that of section 3.2.1 is set up. The IV curve is generated for no photogeneration for both MEDICI and PVSIM. An arbitrary generation rate of $G=1 e^{25} \mathrm{~cm}^{-3} \mathrm{~s}^{-1}$ is introduced. The generation rate occurs uniformly over the entire structure, ie $1 e^{25}$ hole-electron pairs per cubic centimeter are created every second. The results are shown in figures 3.7 and 3.8.

Figures 3.7 and 3.8 show a direct matching of PVSIM results with MEDICI results. 


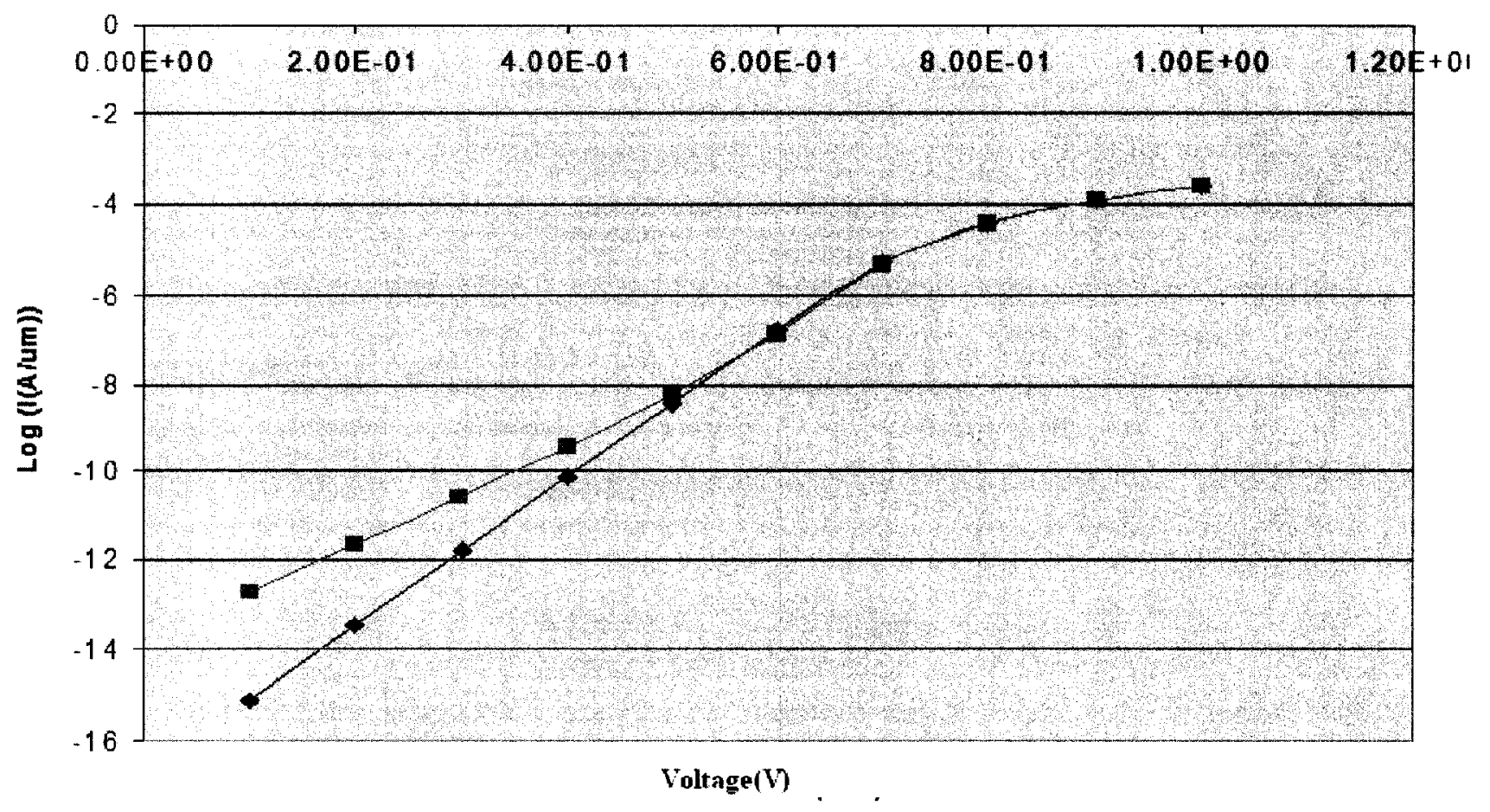

Figure 3.6: Log IV curve results for a 1D PN junction

\subsubsection{Two dimensional $P N$ junction simulations}

Three simulations are performed in two dimensions to verify the functioning of PVSIM in two dimensions. The results are again compared to MEDICI to verify the analysis. All three simulations are performed at zero applied bias voltage. The first simulation is $2 u m x 2 u m$ in size and is discretized into 3600 equal parts, or a 60 by 60 grid. The simulation is run in PVSIM using the $\mathrm{x}$ and $\mathrm{y}$ dimensions of the $3 \mathrm{D}$ simulator. The doping concentrations are the same as in section 3.2.1, except the interface is modified. The interface of $\mathrm{n}$ and $\mathrm{p}$ silicon is changed to a rectangular finger of n-type jutting into the p-type silicon as seen in figure $3.9 \mathrm{~d}$. The finger is of size $30 \mathrm{x} 5$ units where each unit is $2 u m / 60=0.033 u m$ in size. 


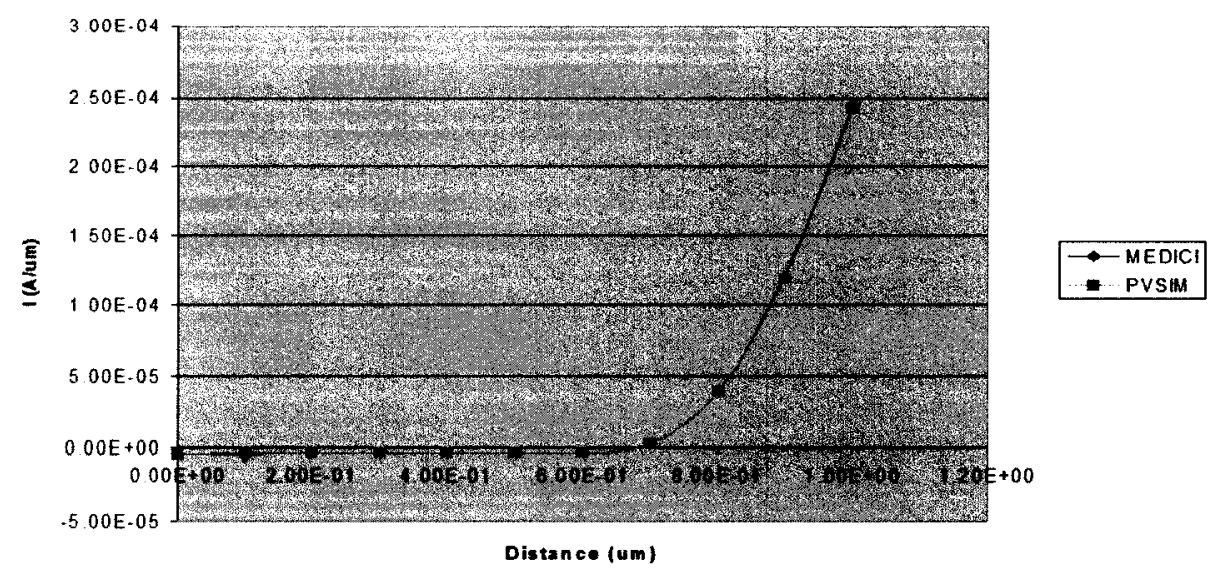

Figure 3.7: IV curve results for a 1D PN junction with photogeneration

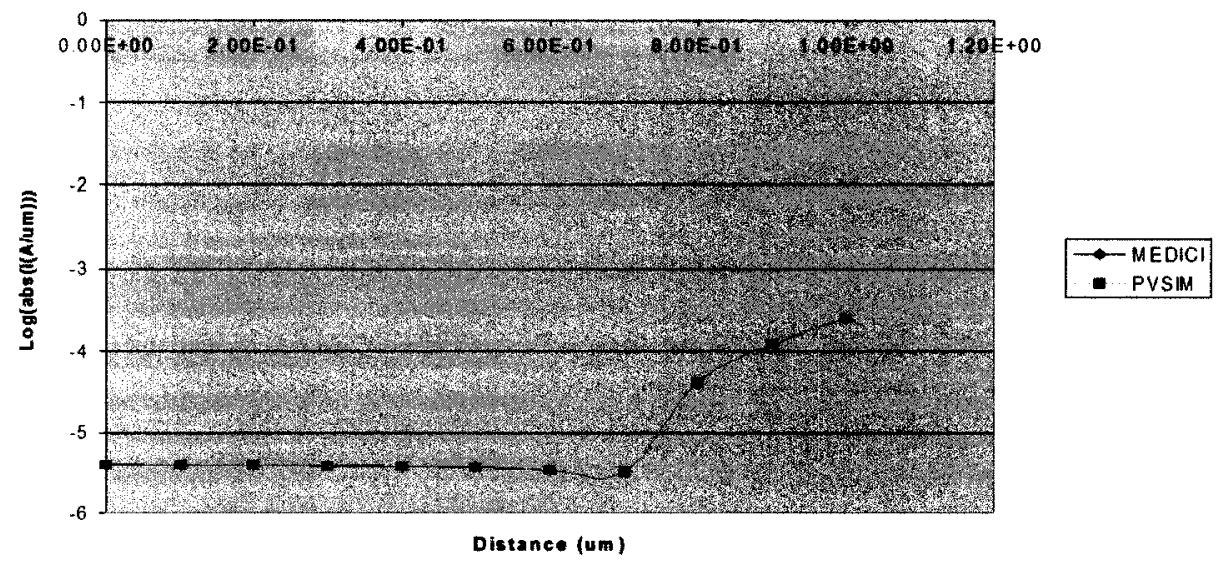

Figure 3.8: Log IV curve results for a $1 \mathrm{D}$ PN junction with photogeneration

Figure $3.9 \mathrm{a}$ shows the carrier concentration in the junction, with figure $3.9 \mathrm{~b}$ the corresponding color legend. Figures $3.9 \mathrm{c}$ and $\mathrm{d}$ show the electron and hole doping as true or false values to illustrate the structure of the device. The second simulation is very similar to the first, except the dimensions are changed to $\mathrm{x}$ and $\mathrm{z}$, and the rectangular structure is changed to $20 \times 20$ units. The results are shown in figure 3.10 .

The third simulation is again similar to the first two. The finger is changed to $15 \times 20$ units and the dimensions are changed to $y$ and $z$. The results are shown in figure 3.11 . 


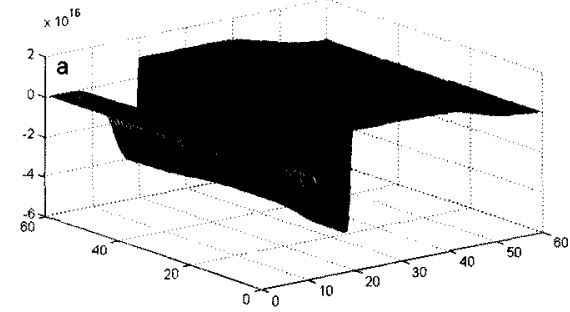

charge carrier density $\left(\mathrm{cm}^{\wedge}-3\right)$

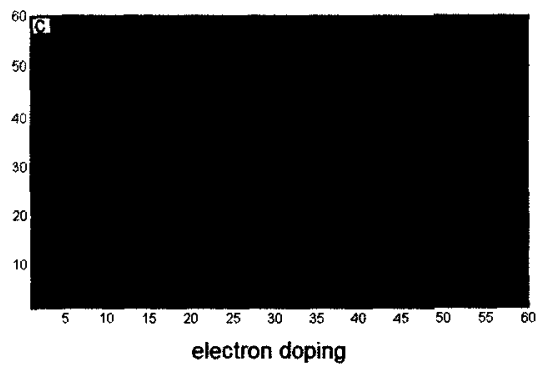

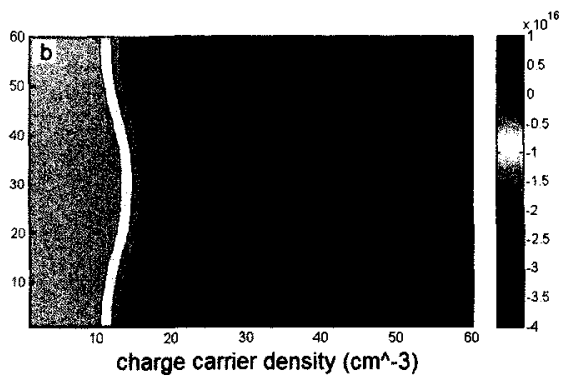

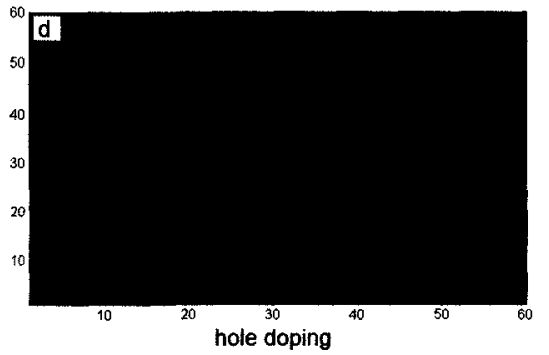

Figure 3.9: PVSIM results for a 2D PN junction with 30x5 unit finger structure

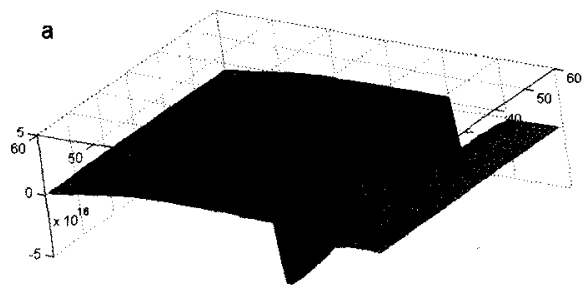

charge carrier density $\left(\mathrm{cm}^{\wedge}-3\right)$

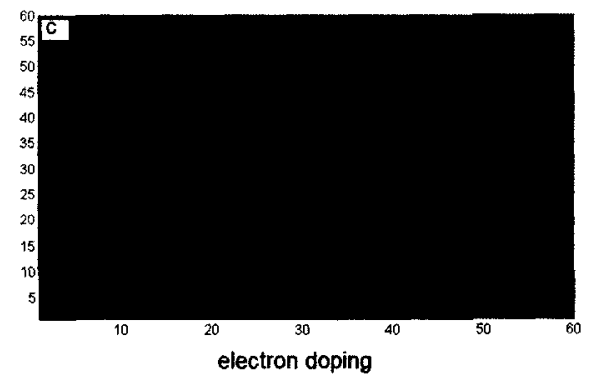

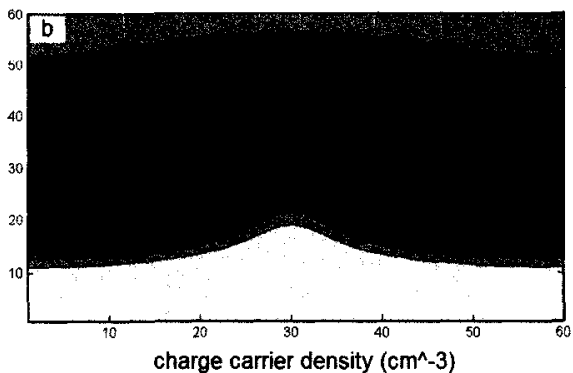

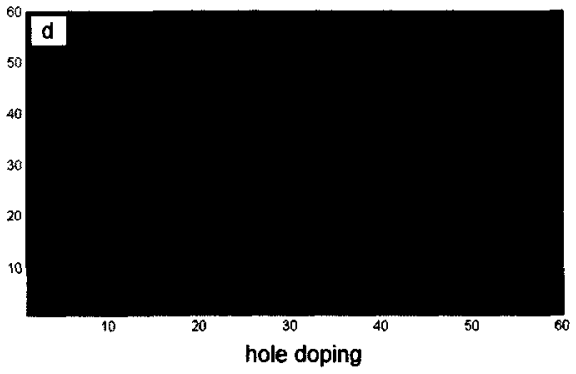

Figure 3.10: PVSIM results for a 2D PN junction with 20x20 unit finger structure 

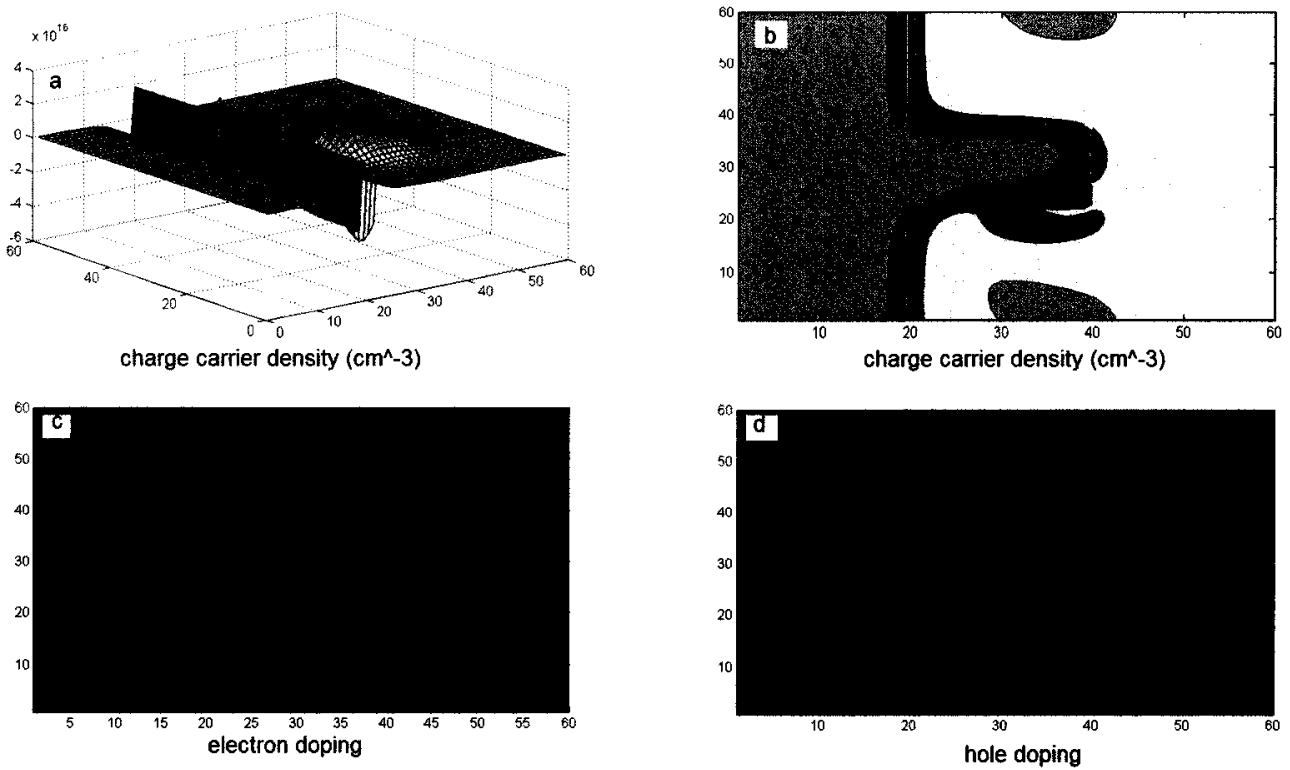

Figure 3.11: PVSIM results for a 2D PN junction with $15 \times 20$ unit finger structure

The same simulations are performed using the $2 \mathrm{D}$ simulation environment in MEDICI. The figures from MEDICI are not shown as the output can not be discerned from the PVSIM results. The carrier concentration results for equilibrium values are subtracted from those produced by MEDICI and the maximum error from each grid is calculated for each simulation. The maximum discrepancy for carrier concentration between the two simulation programs was less than $1^{e} 5 \mathrm{~cm}^{-3}$ for each of the simulations.

\subsubsection{Discussion}

In section 3.2.1, one dimensional results from PVSIM were compared to results obtained by the commercial simulation software MEDICI. The results show that the two simulators produce almost identical results for $1 \mathrm{D}$ simulations given the same input parameters. 
There is a small discrepancy between the results at very small current values. It is unknown whether the error is with PVSIM or MEDICI, as both solutions are approximate solutions solved with numerical methods. The error is very small, and is thought to be due to different number precision storage between the two simulators. MEDICI also uses finite element instead of finite difference method which may cause a slight difference in result. In section 3.2.2, photogeneration is added to the simulations and again the results are compared. The addition of photogeneration in the device yields matching results from the two simulators. In section 3.2.3, the two dimensional simulations run in PVSIM are shown. The results produced by MEDICI match very closely once again.

The results from sections 3.2 .1 to section 3.2 .3 show that the simulation results achieved using PVSIM for 1D, and 2D simulations, as well as photogeneration, are very closely matched to the results from MEDICI. From these results, it can be determined that PVSIM functions properly, and is a good tool for simulating semiconductor devices. The $3 \mathrm{D}$ aspect of PVSIM was not verified, but the 2D results were run in all three dimensions, giving a reasonable idea that the 3D aspect should function properly. 


\section{Chapter 4}

\section{Simulation procedure and parameter implementation}

\subsection{Material parameters}

The simulator requires several material parameters for each material in order to simulate the device. The choice of these parameters is very important as very small changes in the parameters can change the solution dramatically. Materials such as silicon are well studied and the material parameters for silicon are fairly well established and agreed upon. The parameters for titanium dioxide, ITO, and PEDOT are not as well established, and vary from one study to another. The following sections discuss the parameters used in this work, and the reason these values were chosen as being the best available.

\subsubsection{Titanium dioxide}

Titanium dioxide, more well known as titania, can form in various crystalline configurations and exhibit semiconducting properties. Most of the research on the electrical 


\begin{tabular}{|c|c|}
\hline Material Property & Value \\
\hline$\epsilon_{r}$ & 7.6 \\
\hline$U_{n}$ & $0.105 \mathrm{~cm}^{2} / V s$ \\
\hline$U_{p}$ & $0.3 \mathrm{~cm}^{2} / V s$ \\
\hline$D_{n}$ & $0.003 \mathrm{~cm}^{2} / \mathrm{s}$ \\
\hline$D_{p}$ & $0.009 \mathrm{~cm}^{2} / \mathrm{s}$ \\
\hline$n_{i}$ & $3.0^{10} \mathrm{~cm}^{-3}$ \\
\hline
\end{tabular}

Table 4.1: Material parameters for rutile titania

properties of titania are limited to the study of rutile titania as it shows good $\mathrm{n}$ and $\mathrm{p}$ type properties [18-20]. The material parameter values used for rutile titania are shown in table 4.1. All values are for a steady temperature of $300 \mathrm{~K}$.

The values for the hole and electron mobility are determined from Nowotny el al [21]. Nowotny et al take values reported in the literature and attempt to fit the data with defect chemistry defect models. The study shows a recent and up to date analysis of the available data and excludes outdated studies and those shown to be obsolete. The diffusion constants are determined from equation 2.1.8.

The intrinsic density or intrinsic electronic equilibrium of titania was found again from Nowotny el al [22]. There was only one study found for the study of the intrinsic density of titania. The bandgap of titania is large in comparison to typical semiconductors, and so the intrinsic density is very small.

The permittivity of titania is found to vary greatly according to the type of crystal structure. The relative permittivity in titania particles in conducting polymer is reported to be extremely high [23] whereas the same parameter is reported to be a factor of ten lower in nanostructured and porous titania $[24,25]$. This work will use more conventional 
values used for solid rutile titania [26]. It is believed that the reported values for structured titania and polymer embedded titania are influenced by the permittivity of the surrounding materials. The simulation will therefore use base values for solid titania.

\subsubsection{Indium Tin Oxide}

Indium tin oxide is a heavily doped n-type semiconductor exhibiting both high transparency in the visible spectrum and very high conductivity [27]. It is most commonly considered as indium oxide with a tin doping $\left(\mathrm{In}_{2} \mathrm{O}_{3}: \mathrm{Sn}\right)$ but can equally be considered as tin oxide with an indium doping $\left(\mathrm{SnO}_{2}: \mathrm{In}\right)$. ITO films are available commercially with a given sheet resistivity specified by the supplier. From the value of resistivity, the values for mobility should be easily determinable. However, the manufacturers of ITO do not regulate specific carrier concentrations, and simply vary the film in order to achieve the desired sheet resistivity [28].

The bandgap of ITO is directly related to the carrier concentration, or tin doping concentration, in the film. The relation between the parameters and carrier concentration is shown in figures 4.1 to 4.3 from Nagatomo et al [29].

To determine the electrical properties of ITO, an arbitrary doping must be chosen, and the other values calculated subsequently. The doping is chosen to be $N_{d}=9.47 e^{20} \mathrm{~cm}^{-3}$, and the values for permittivity and mobility interpolated from the data provided by Nagatomo et al [29] and the use of equation 4.1.1 for conductivity. The diffusion constants are again calculated using equation 2.2.2. 


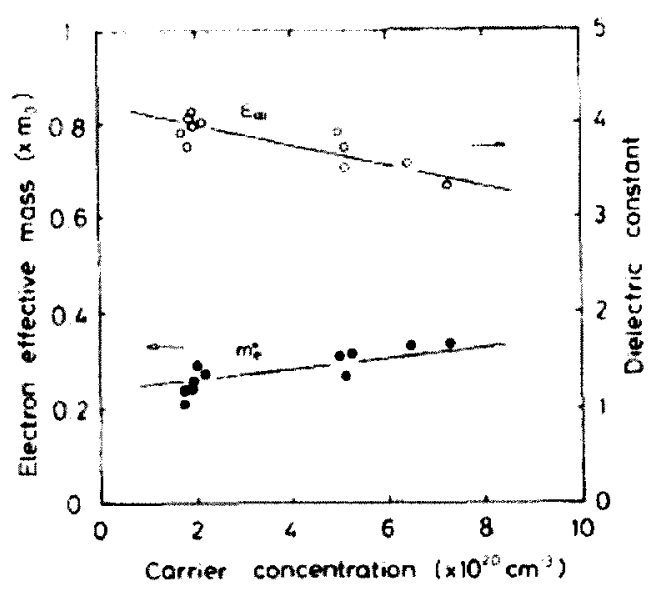

Figure 4.1: Permittivity and effective electron mass of ITO as a function of carrier concentration

\begin{tabular}{|c|c|}
\hline Material Property & Value \\
\hline$\epsilon_{r}$ & 8.9 \\
\hline$U_{n}$ & $46.5 \mathrm{~cm}^{2} / V s$ \\
\hline$U_{p}$ & $0.1 \mathrm{~cm}^{2} / V s$ \\
\hline$D_{n}$ & $1.2 \mathrm{~cm}^{2} / \mathrm{s}$ \\
\hline$D_{p}$ & $0.0025 \mathrm{~cm}^{2} / \mathrm{s}$ \\
\hline$N_{d}$ & $9.47 e^{20} \mathrm{~cm}^{-3}$ \\
\hline
\end{tabular}

Table 4.2: Material parameters for ITO

$$
\sigma=\left(p \mu_{p}+n \mu_{n}\right) q
$$

Table 4.2 shows the final parameters used for ITO at at temperature of $300 \mathrm{~K}$. The mobilities of ITO are very low, as well as the diffusion constants, implying that the substance is not a good conductor. However, the doping is extremely high, and so the resistivity of the film is very low. 


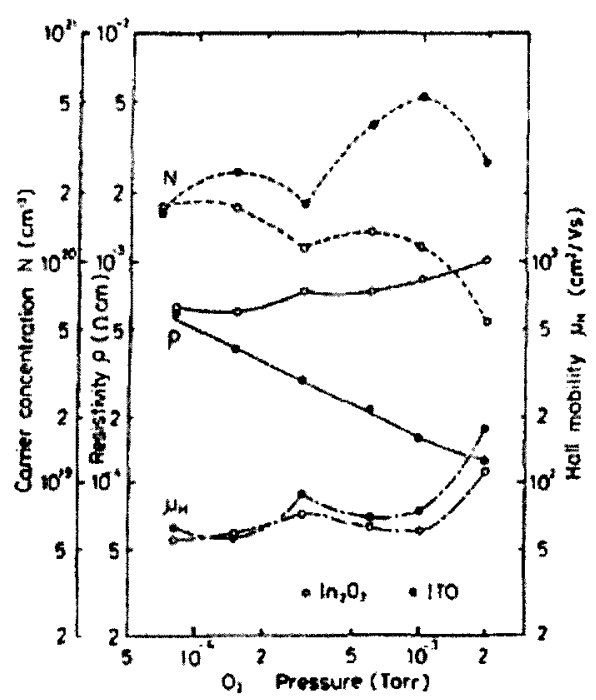

Figure 4.2: Hall mobility and resistivity of ITO as a function of carrier concentration

\subsubsection{PEDOT}

Poly(3,4-ethylenedioxythiophene), more well known as simply PEDOT, is a conducting polymer well know for its conductivity, transparency and stability. PEDOT is readily water soluble, which allows many options for deposition. The PEDOT can deposited in liquid format, allowing the liquid to fill in the spaces between the titania structure. The substance cures to a solid, flexible film.

PEDOT is a conducting polymer. The charge transportation mechanisms in semiconductors are different to those involved in polymers. Whereas charge carriers in semiconductors are governed by drift and diffusion, those in conducting polymers are governed by charge hopping and polymer chain diffusion [30]. Ideally, PVSIM would be modified to more accurately describe the charge transport. For simplicity, the simulator treats PEDOT as a semiconductor.

PEDOT contains HOMO and LUMO levels at $5.2 \mathrm{eV}$ and $3.5 \mathrm{eV}$ [31]. The levels are 


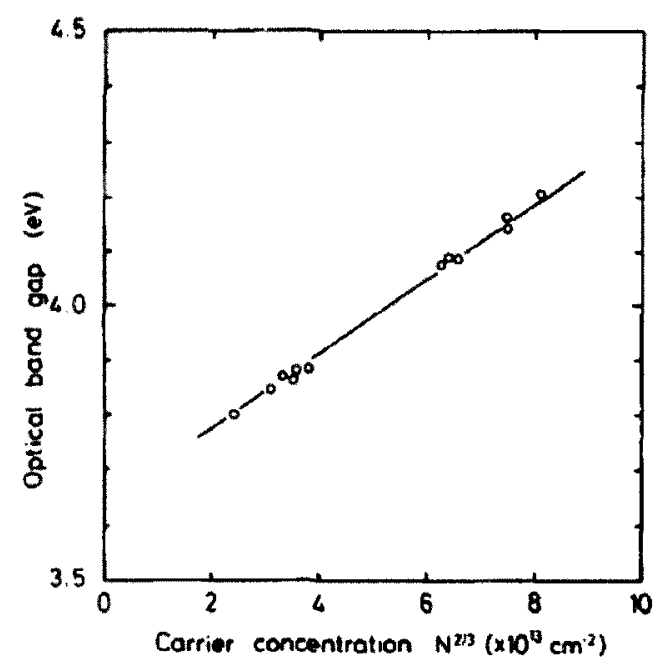

Figure 4.3: Optical bandgap of ITO as a function of carrier concentration

\begin{tabular}{|c|c|}
\hline Material Property & Value \\
\hline$\epsilon_{r}$ & 4.0 \\
\hline$U_{n}$ & $5.12^{-5} \mathrm{~cm}^{2} / V s$ \\
\hline$U_{p}$ & $2.3^{-8} \mathrm{~cm}^{2} / V s$ \\
\hline$D_{n}$ & $1.3^{-6} \mathrm{~cm}^{2} / \mathrm{s}$ \\
\hline$D_{p}$ & $6^{-10} \mathrm{~cm}^{2} / \mathrm{s}$ \\
\hline$N_{a}$ & $2.70^{24} \mathrm{~cm}^{-3}$ \\
\hline
\end{tabular}

Table 4.3: Material parameters for PEDOT

similar to conduction and valence band edges in semiconductors. Although not analogous, the PEDOT/semiconductor boundary is very similar to a semiconductor junction. The HOMO and LUMO levels are taken in PVSIM as the bandgap. Along with experimental values for mobility [30,32], and for conductivity [33], a model is determined to model PEDOT as a semiconductor. The conducting polymer PEDOT is modeled as a P-type semiconductor with high p doping. The values used in PVSIM for PEDOT are shown in figure 4.3 . 


\subsubsection{Ruthenium Dye N3 and generation rate modeling}

The generation in DSSC's occurs in the dye, and the charge is then transported by the semiconductor structure. Once the electron-hole pair is generated by the dye, the charge must be swept away as quickly as possible before the charges recombine. It is important to choose a dye that works best with the given semiconductor in the photovoltaic device.

The ruthenium N3 dye is commonly used in photovoltaic devices using $\mathrm{TiO} 2$ substrates $[34,35]$. It is used commonly simply because it gives good results for both dye coverage and quantum efficiency. A density functional theory(DFT) calculation was performed by Perrsonet et al [36] of a titania crystal sensitized by a ruthenium N3 dye molecule. The density of states function of the combined $\mathrm{TiO} 2 / \mathrm{N} 3$ and of $\mathrm{N} 3$ alone are shown in figure 4.4 .

Figure 4.4 shows the lowest few unoccupied dye orbitals are close in energy to the $\mathrm{TiO} 2$ conduction band edge. The authors believe that the strong couplings between the $\mathrm{TiO} 2$ substrate and the dye states correspond to injection times in the order of $10 \mathrm{fs}$ [36]. Compared to recombination times in the order of 1us, the injection is almost instantaneous.

Due to the very quick injection of the charge carriers from dye to titanium, the dye is not simulated with PVSIM. The simulation assumes the charge is generated at the TiO2/PEDOT junction, and that 100 percent of the charge generated is injected into the titania.

Lu et al [37] measured the absorption spectra of N3 dye, and the results are shown in figure 4.5. 

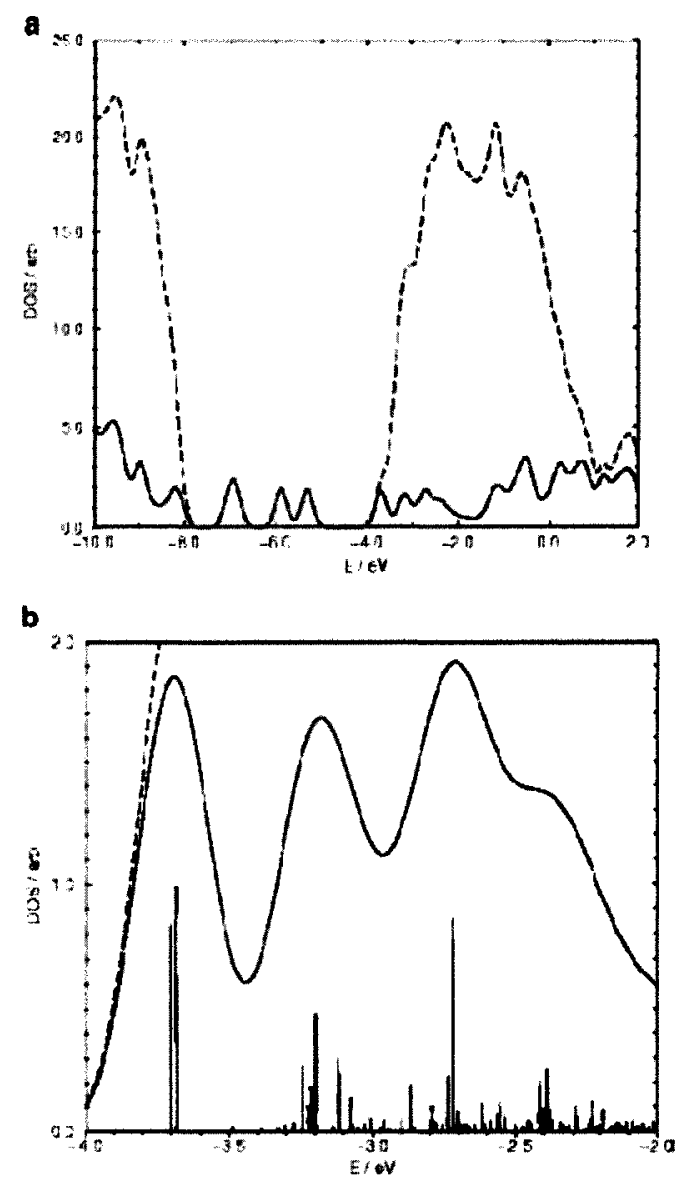

Figure 4.4: Calculated density of states (DOS) of N3 attached to a TiO2 nanocrystal (a) Total DOS (dashed curve) and N3 PDOS (solid curve). (b) Magnified view of the DOS near the conduction band edge

It is assumed that photovoltaic devices are for use out doors, where they will absorb natural light from the sun. The incident spectrum used to evaluate solar cell performance is the American Society for Testing and Materials (ASTM) Terrestrial Reference Spectra for Photovoltaic Performance Evaluation, also known as Air Mass 1.5 or AM1.5. The conditions selected were considered to be a reasonable average for the 48 continguous states of the United States of America (U.S.A.) over a period of one year. The tilt angle selected is approximately the average latitude for the contiguous U.S.A. Figure 4.6 shows 


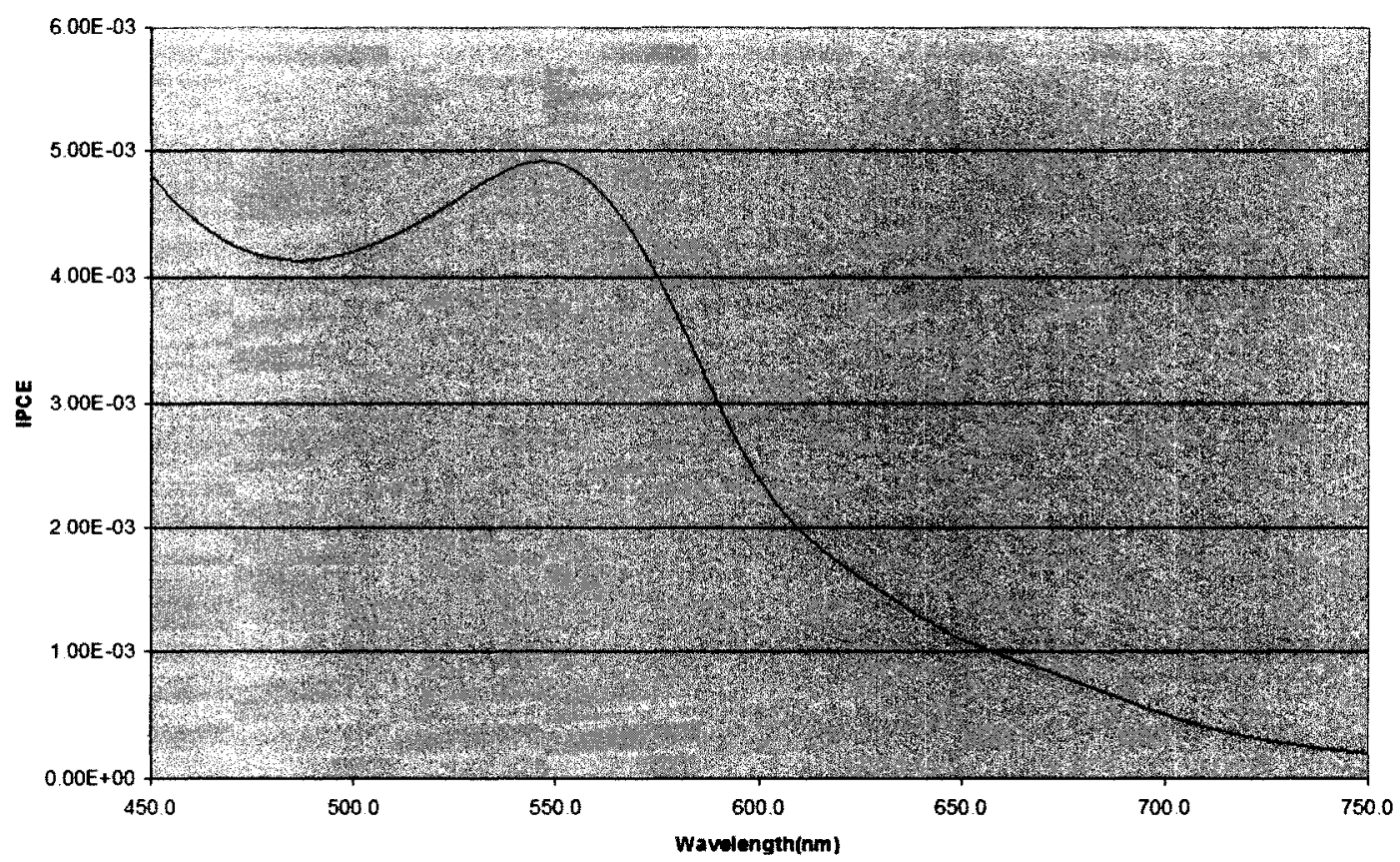

Figure 4.5: Absorption spectrum of the N3 Ruthenium dye on TiO2 substrate

a chart of spectral irradiance standard AM1.5.

To convert spectral irradiance to photon incidence, equation 4.1.2 is used. I represents photon incidence, Ir spectral irradiance and t spacing. Photon incidence is shown in figure 4.7.

$$
I\left(n m^{-1}\right)=\operatorname{Ir}\left(\frac{J}{m^{2} n m s}\right) t(1 n m) \frac{\lambda(n m)}{1240 \mathrm{eVnm}} \frac{1 \mathrm{eV}}{1.602 \mathrm{e}^{-19} \mathrm{~J}}
$$

The photon incidence is multiplied by the dye absorbtion spectrum to determine the photon absorption from the dye at AM1.5 conditions. The results are shown in figure 4.8. The values from the photon absorption spectrum are added together to determine a total photon absorption rate at AM1.5 of $2.81^{14}$ photons per square centimeter per second. 


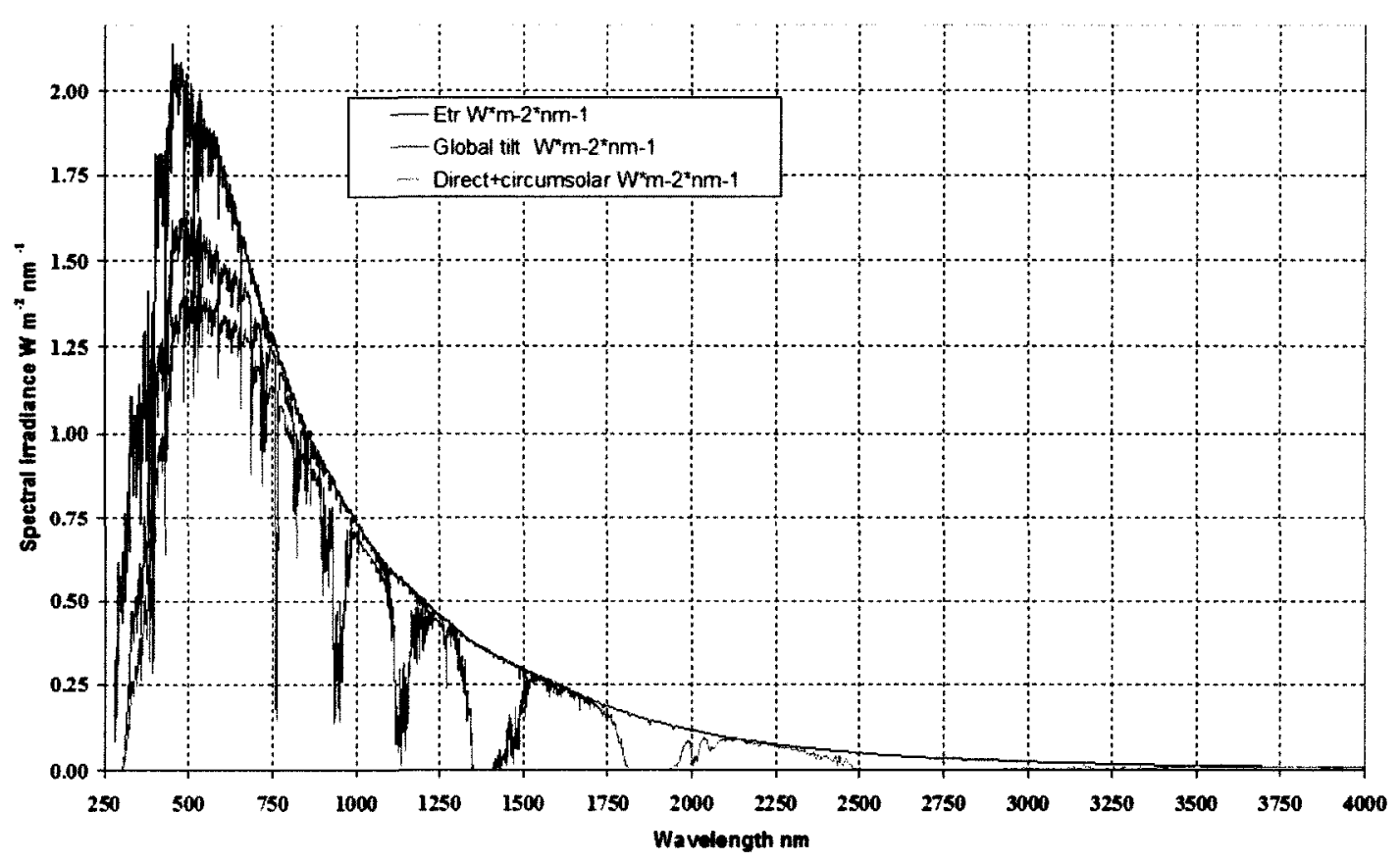

Figure 4.6: AM1.5 Spectral irradiance

\subsection{Simulation procedure}

The simulation program design has been reviewed, and the parameters for each material determined. In order to simulate the photovoltaic cell, several other pieces of information are required. The structure of the device must be input, and a initial guess must be determined. The following sections review the simulation set-up through input parameters and a simulation flow of a sample device to illustrate the initial guess procedure.

\subsubsection{Simulation set-up}

The simulation begins with a set of inputs. The input cube is discretized in $\mathrm{N}$ parts, with LY, LZ, and LX the number of divisions in the Y, Z, and X dimensions. The size of each discretization is input parameter h. Along with these input parameters, two matrices 


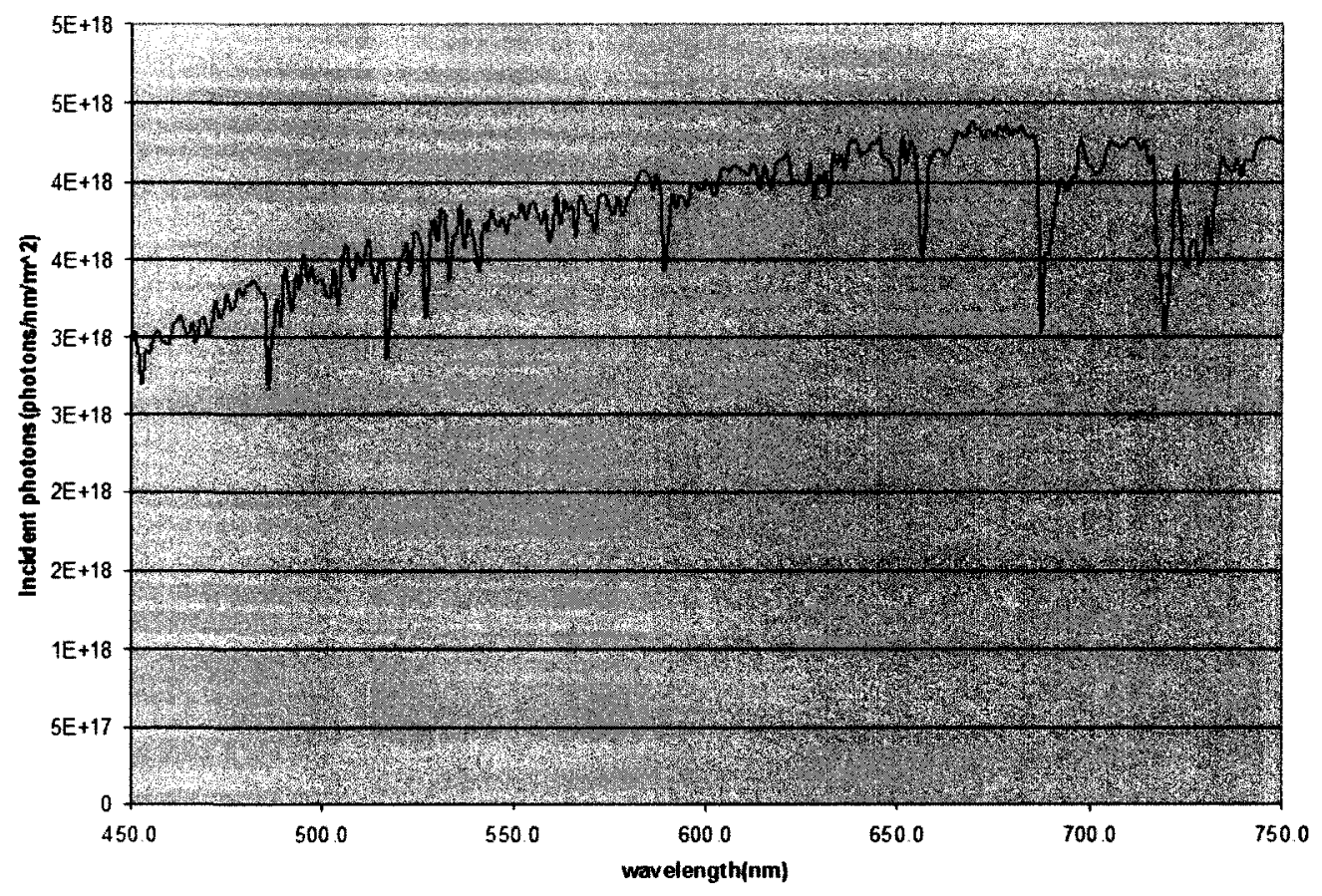

Figure 4.7: AM1.5 Photon incidence

containing the structure of the titanium, and the generation location are included. A sample of the structure and generation are shown in figure 4.9. The structure matrix is composed of the numbers 1, 2 and 3 which represent PEDOT, TiO2 and ITO respectively. The generation matrix represents the location of generation by 1 or 0 .

An input file uses the information from the input parameters and writes a file 3DMatFile.mat. This file contains matrices for the entire structure containing all the information needed for simulation. These include;

NA- Acceptor doping

ND- Donor doping

DN- Electron diffusion constant 


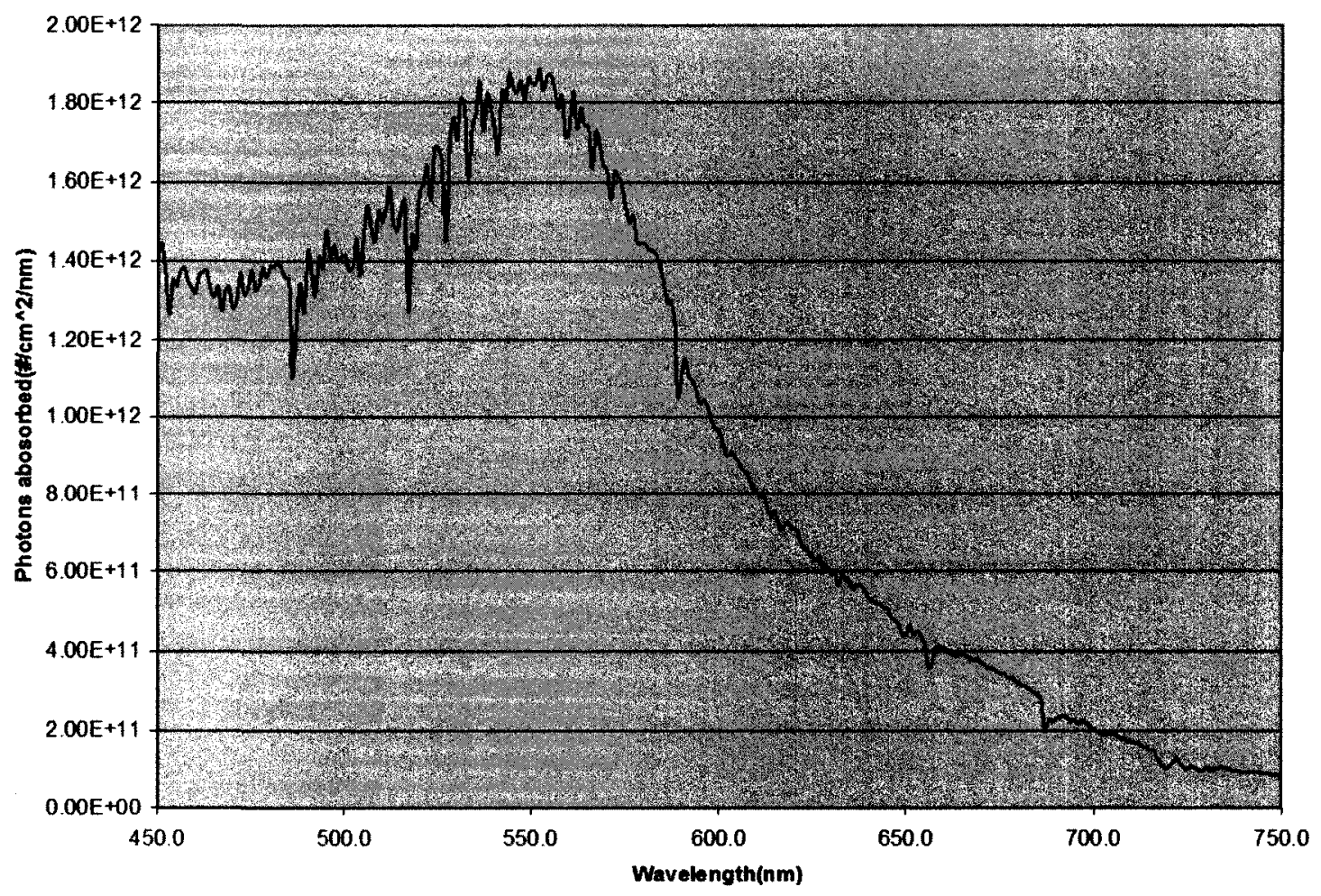

Figure 4.8: Dye photon absorption at AM1.5

DP- Hole diffusion constant

UN- Electron mobility constant

UP- Hole mobility constant

ER- Relative permittivity

CLOW- Boundary conditions at minimum

CHIGH- Boundary conditions at maximum

$\mathrm{N}$ - Initial guess for $\mathrm{N}$

$\mathrm{P}$ - Initial guess for $\mathrm{P}$

V- Initial guess for $\mathrm{V}$ 


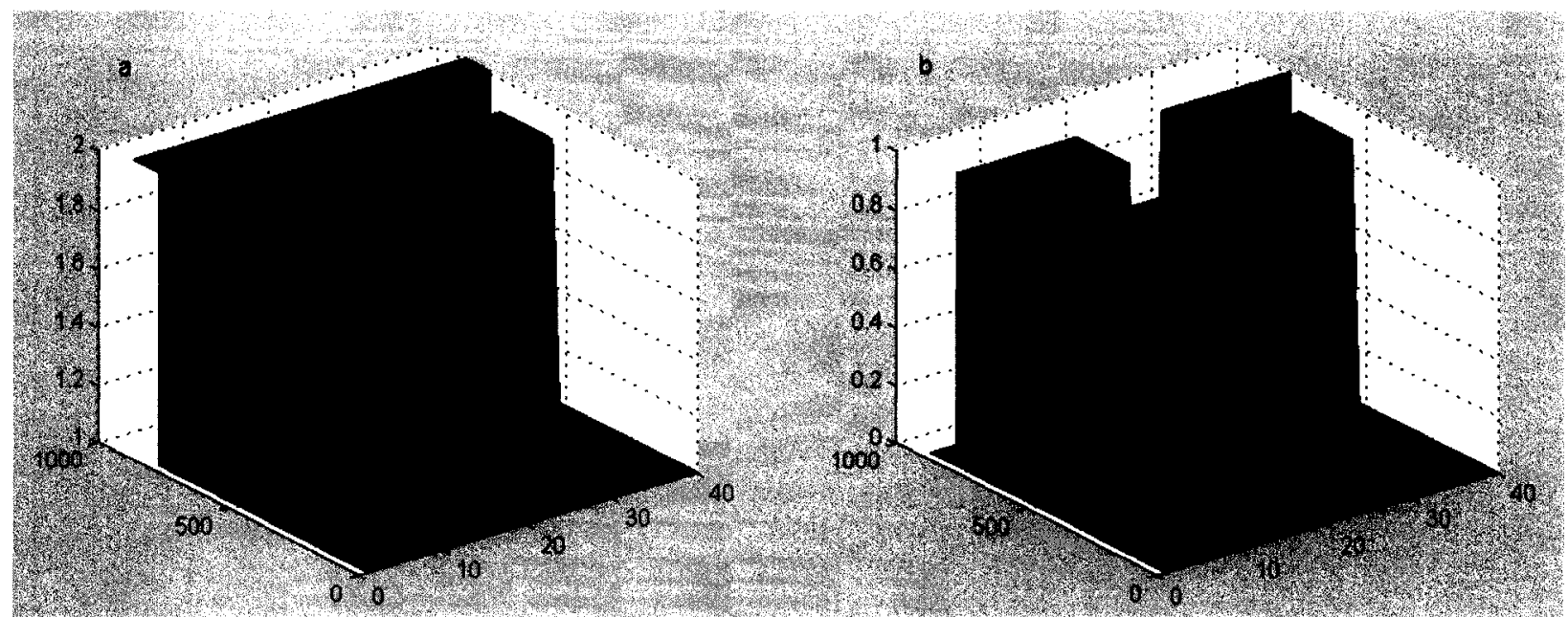

Figure 4.9: (a) Structure of titania (b)Generation input

The initial guess for a single junction device uses the equilibrium values found in equations 2.3.1 and 2.3.2, and a zero potential across the device. However, when dealing with multiple junctions, this technique does not produce convergence. Each junction must first be simulated separately, and then ramped up to zero current conditions. The two junctions can then be assembled and simulated.

\subsubsection{Simulation flow}

The following section discusses the steps involved in simulating a two junction structure of any type. The structure used as an example here is a two dimensional structure with a total length of $15 \mathrm{~nm}$, divided into 1200 units in the $\mathrm{x}$-direction and 1 unit in the $\mathrm{y}$ direction. This is essentially a 1D structure, but the methodology is identical for a two dimensional structure. The structure is made of three materials, ITO from units 1 to 200 , TIO2 from units 201 to 1000 and PEDOT from 1001 to 1200 . The generation when added occurs in unit 1000, on the PEDOT, TIO2 boundary. A pictorial view of the structure is 
shown in figure 4.10 .

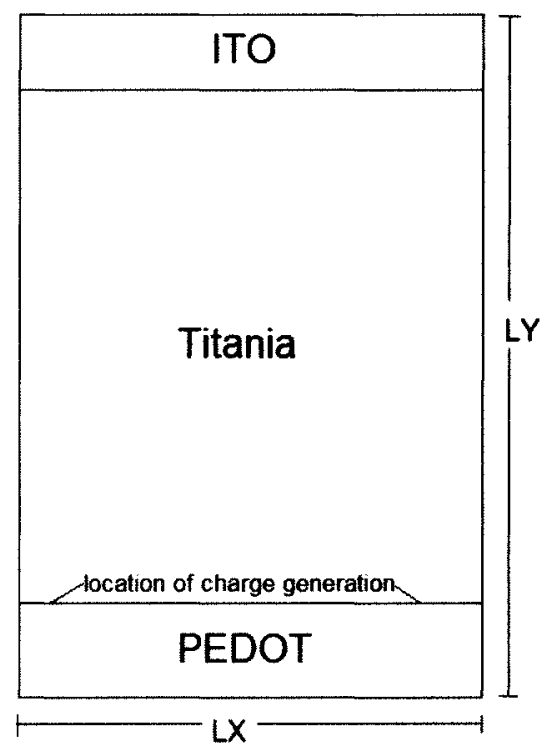

Figure 4.10: Pictorial view of simulation

The structure is first broken into two parts for simulation, a Part A from units 0 to 300 and a Part B from units 301 to 1200 . This is done so each junction can be simulated separately. Part A is simulated first at a bias of zero volts, and then the bias voltage is ramped up in $0.1 \mathrm{~V}$ increments. The IV curve for Part $\mathrm{A}$ can be seen in figure 4.11. The zero current bias voltage is determined to be $3.0 \mathrm{~V}$, and so the solution at this voltage is saved for future use. The charge carrier concentration for the junction can be seen in figure 4.12. The depletion region can be clearly seen at the ITO/TiO2 junction.

Part $\mathrm{B}$, the TiO2/PEDOT junction is simulated at zero bias voltage, and then the bias is ramped up in $0.1 \mathrm{~V}$ increments. The zero-current bias voltage is determined to be 0.7V. Figure 4.13 shows the carrier concentration across the junction. Again a depletion region can be seen, however it is very small due to the large difference in doping and 


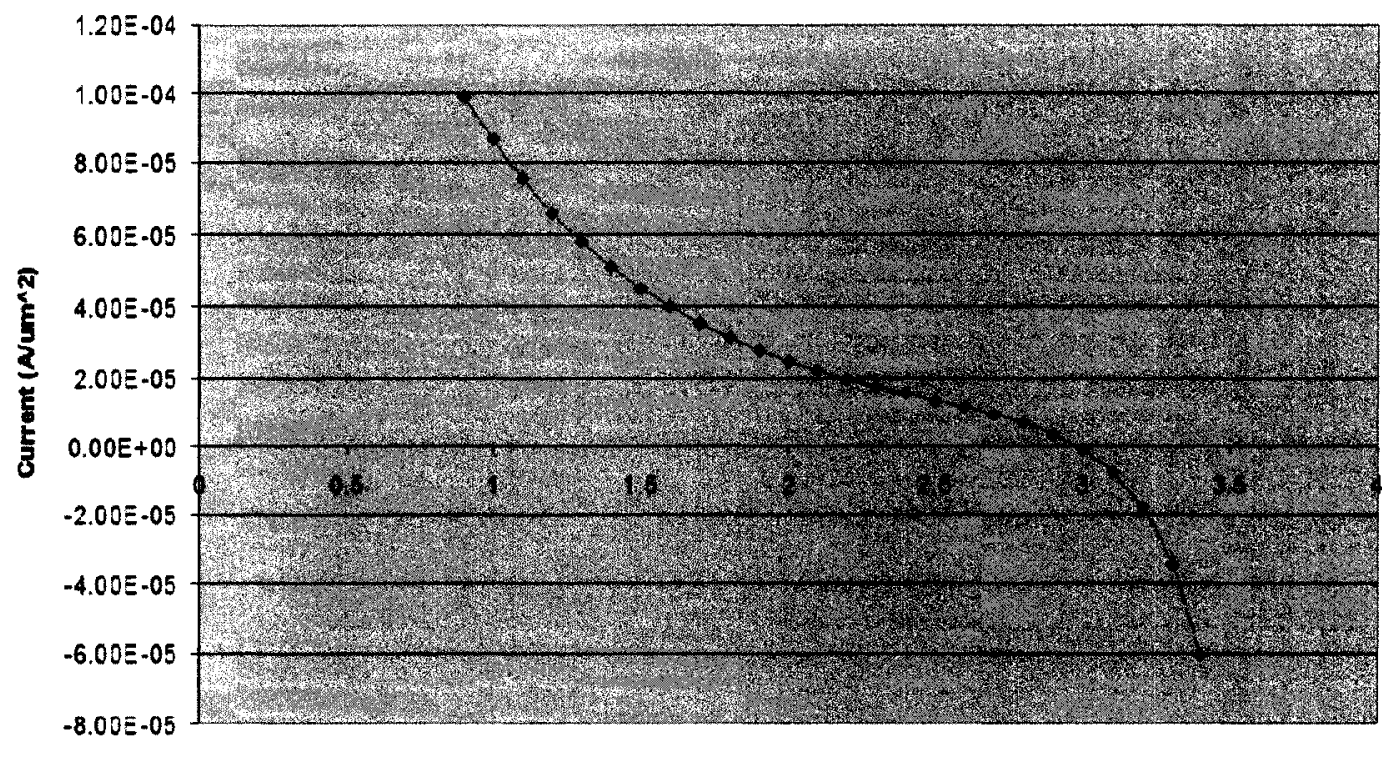

Voltage (V)

Figure 4.11: IV curve for ITO/Titania junction in one dimension

diffusion coefficients of the two materials.

The solution of Part $\mathrm{A}$ at $3.0 \mathrm{~V}$ and the solution to Part $\mathrm{B}$ at $0.7 \mathrm{~V}$ are then amalgamated. The program combines the solutions of the two junctions for voltage, charge, $\mathrm{n}$ and $\mathrm{p}$ concentrations. The combined solution is then simulated at $3.7 \mathrm{~V}$ bias and ramp down and up in $0.1 \mathrm{~V}$ increments to determine the IV curve. The IV curve is shown in figure 4.14. The zero current bias voltage is determined to be $3.47 \mathrm{~V}$. The carrier concentrations in the device are shown in figure 4.15. The two junctions and depletion areas can be clearly seen.

The zero current bias voltage is subtracted from the bias voltages in the IV curve to determine the applied voltages on the device. The IV curve with applied voltage is then produced. A generation rate of $10^{30} \mathrm{~cm}^{-3} \mathrm{~s}^{-1}$ is added, and the structure is again simulated over a range of voltages. The two IV curves are compared in figure 4.16 . The 


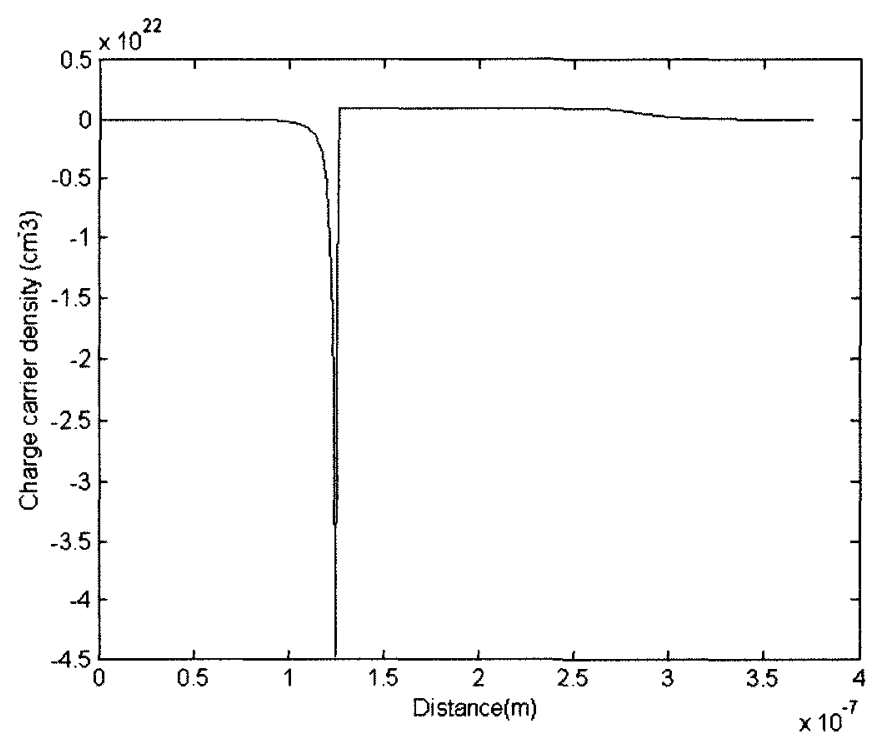

Figure 4.12: Carrier concentration for 1D ITO/Titania junction

power of the device is then calculated by multiplying the voltage by the negative current produced. The efficiency in the results section refers to the total percent of power that is converted from generation charge to electrical output. The efficiency is very different from light harvesting efficiency, which refers to percent of light converted to electrical power. Not all the light is absorbed in a given device, and so there is losses converting from light to generation which is not calculated here. Figure 4.17 shows the efficiency versus voltage plot for the example structure.

All the structures in the results section are simulated with the methodology discussed in this section. The methodology is designed to eliminate non-convergence in the NewtonRaphson algorithm. The ITO/TiO2 junction need only be simulated once for structures with the same total cubic size, and ITO junction location. The TiO2 structure is then modified, simulated as a junction with PEDOT and the results combined. 


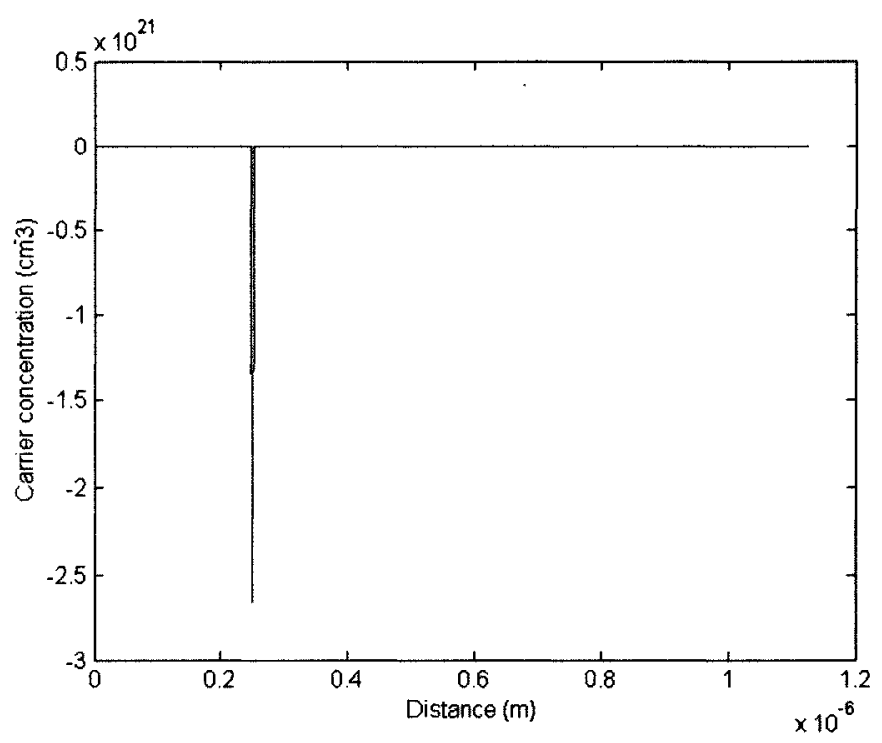

Figure 4.13: Carrier concentration for 1D Titania/PEDOT junction

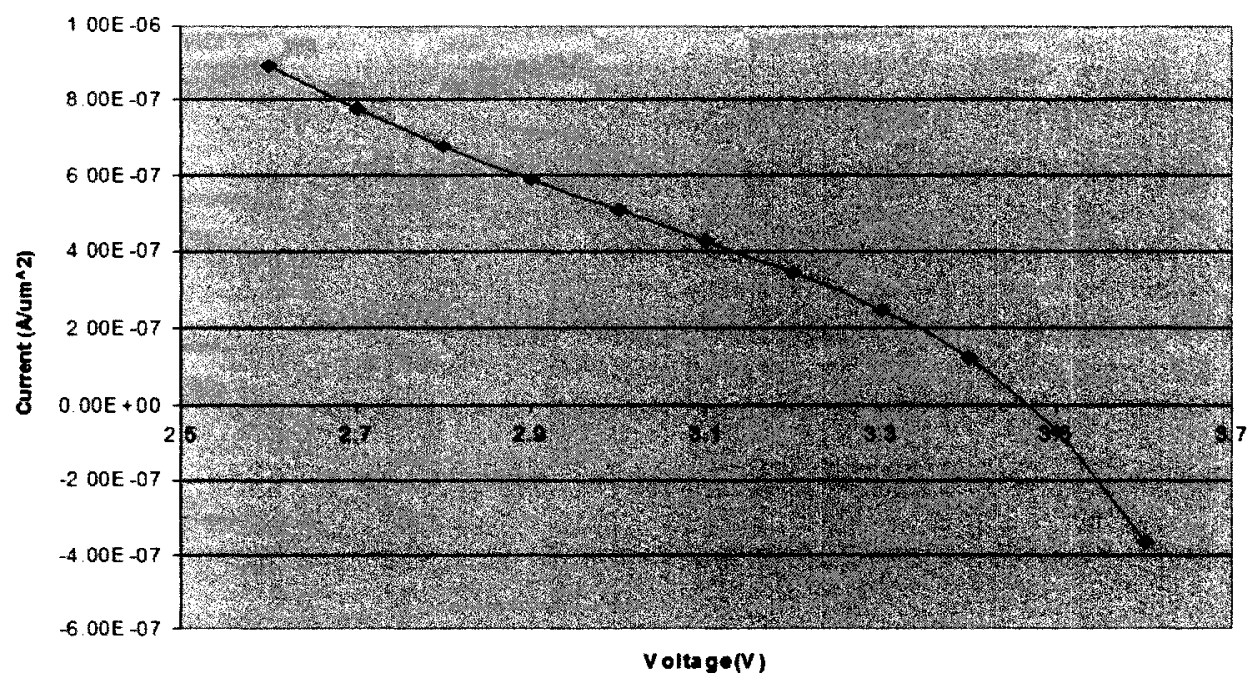

Figure 4.14: IV curve for 1D ITO/Titania/PEDOT device 


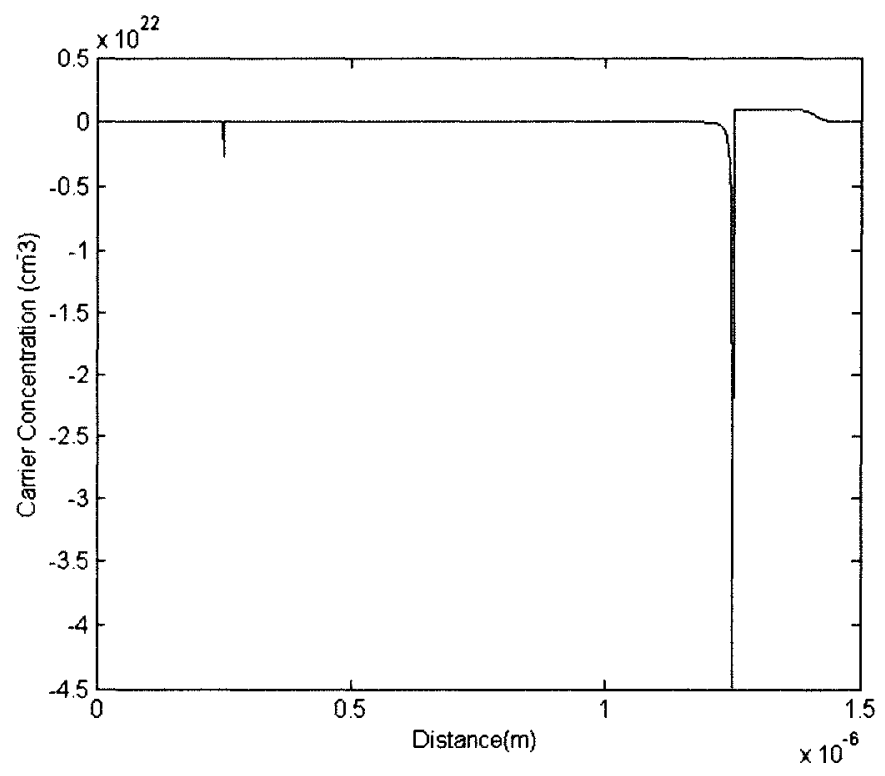

Figure 4.15: Carrier concentration for 1D ITO/Titania/PEDOT device

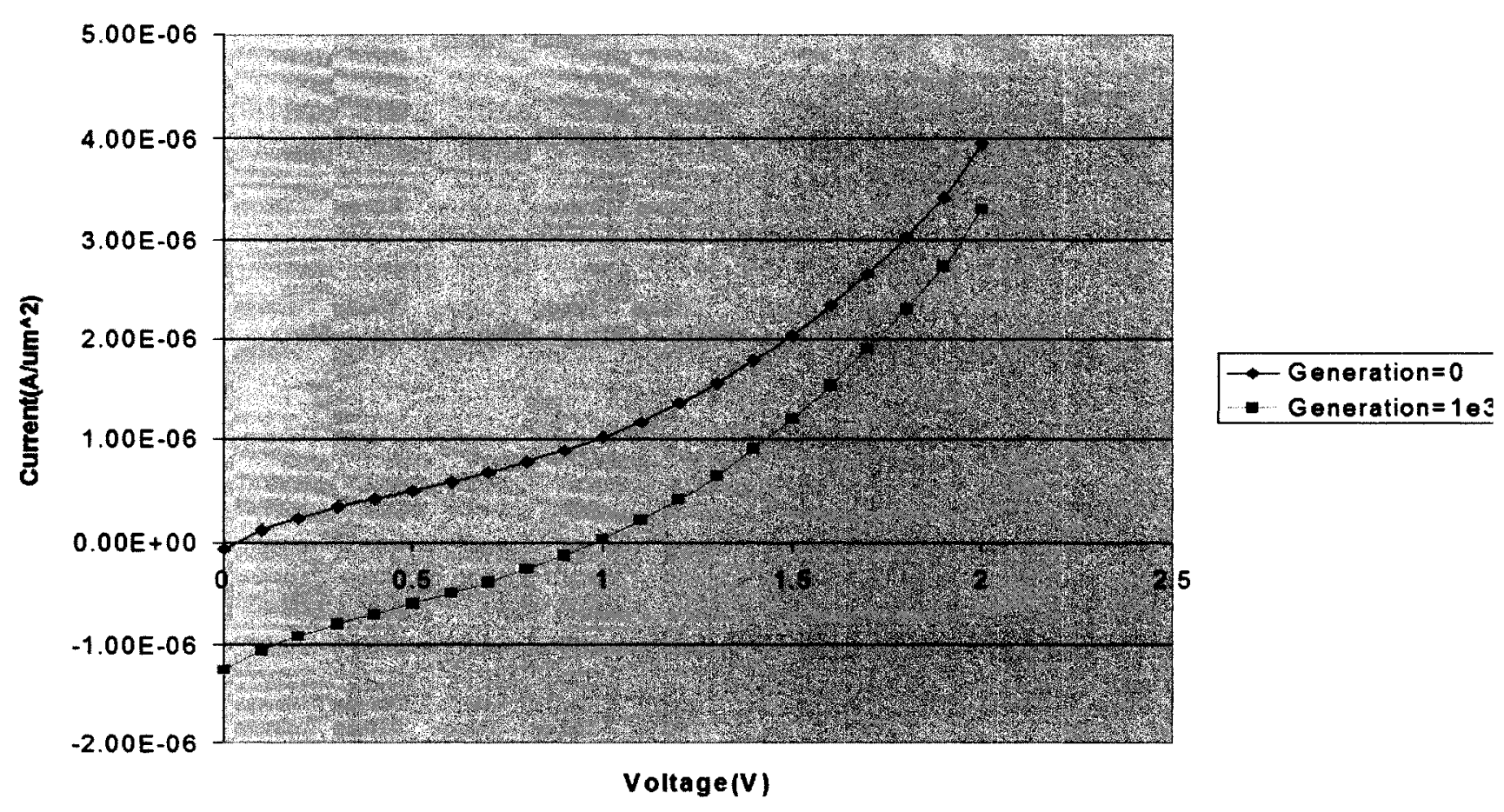

Figure 4.16: Applied voltage IV curve for 1D ITO/Titania/PEDOT device 


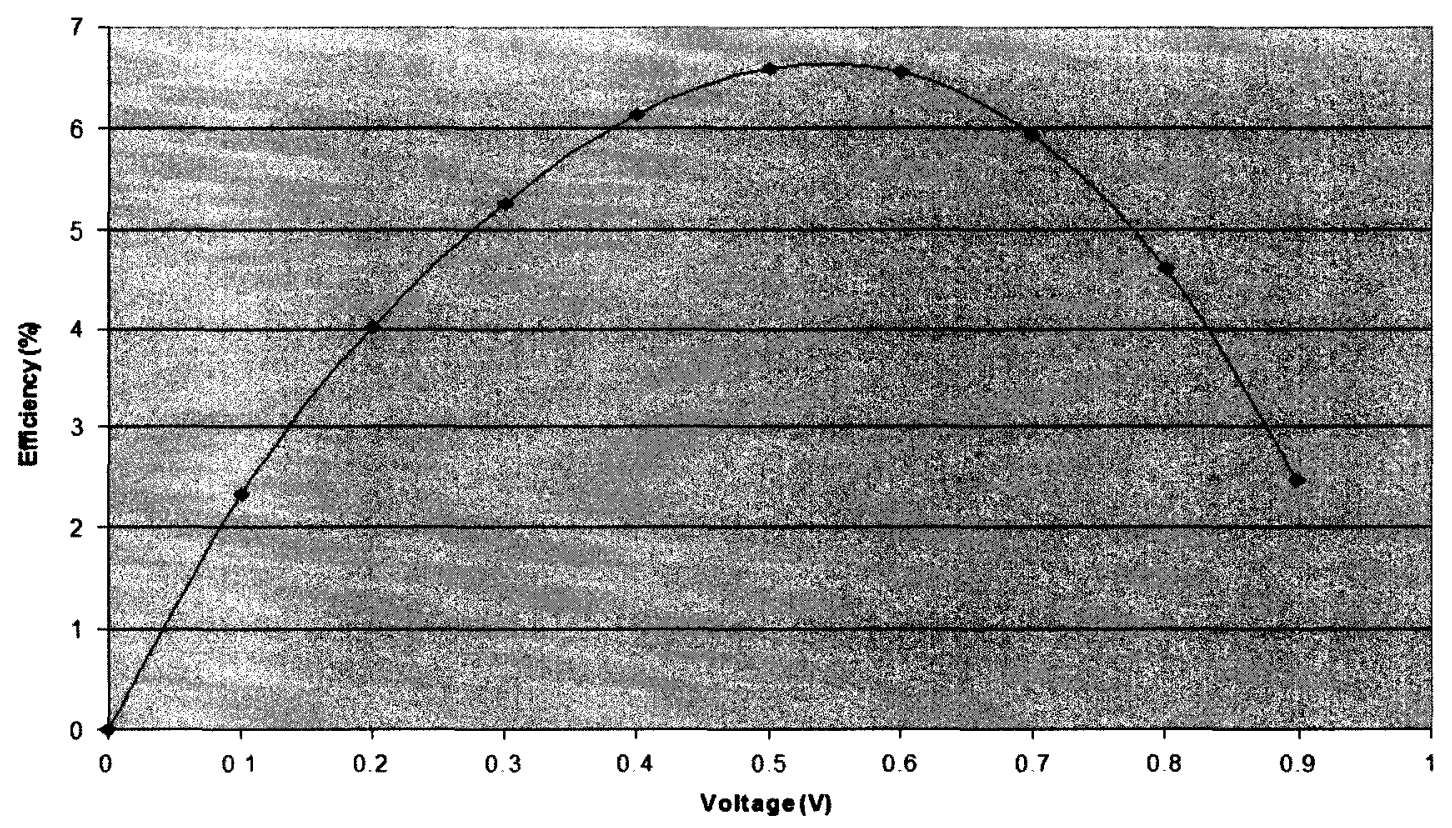

Figure 4.17: Cell efficiency for sample structure 


\section{Chapter 5}

\section{Results}

In this chapter, the results obtained from simulations using PVSIM are reviewed. The first section discusses the baseline results achieved for a ITO/TiO2/PEDOT device with no structure. The baseline results determine the parameters for the subsequent structured devices. The second section reviews the results obtained for the structure devices, including both the simple finger structures and the more complex structures.

\subsection{Baseline results}

The baseline structures are formed of one layer of ITO, one layer of titania, and one layer of PEDOT, one on top of the other. A number of simulations were performed to determine the best thickness of each layer, as well as the element size that is needed to produce good results. The PEDOT and ITO layers were started very small and were increased in size until no discernible difference in results was seen. It was determined that no more than 5nm thickness is needed for both ITO and PEDOT. The element size was also decreased until the results converged to an equivalent result. The minimum element 
size was determined to be $0.0125 \mathrm{~nm}$. The very small element size and the relatively small outside layers are a direct consequence of the very shallow depletion layers in both junctions.

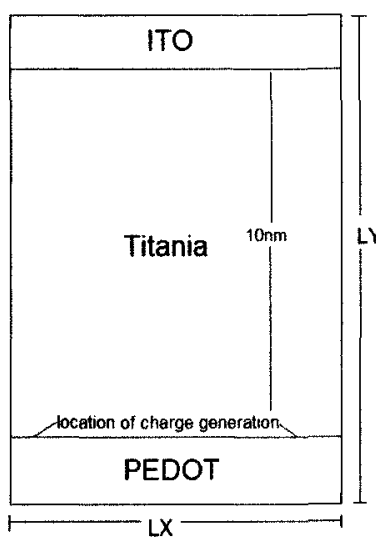

A

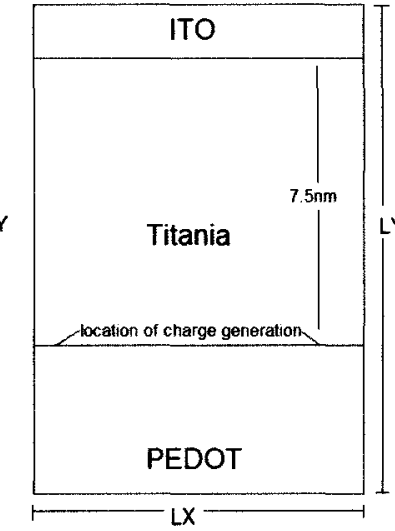

B

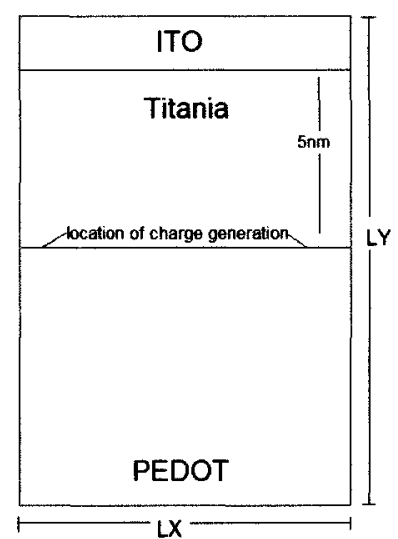

$\mathrm{C}$

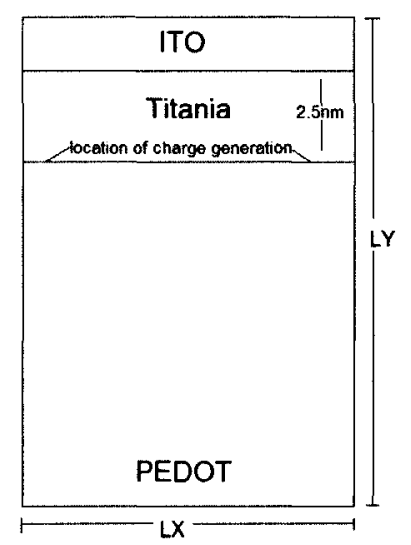

$\mathrm{D}$

Figure 5.1: Pictorial view of baseline structures

The thickness of the titania layer was modified, and the results recorded to note the effect of titania thickness on efficiency. The structures are shown in figure 5.1 with the varying titania thicknesses denoted. The IV curves of the varying thickness devices are shown in figure 5.2 and the efficiency curves for these devices in figure 5.3.

The generation rate for the devices as determined in section 4.1.4 for AM1.5 conditions is $2.81^{14}$. Since the generation is modeled as occurring in only one layer of elements, the corresponding generation rate for element size of $0.0125 \mathrm{~nm}$ is $2.25 \mathrm{e}^{23} \mathrm{~cm}^{-3}$. When the flat structures are exposed to the calculated generation, no power is generated, and thus zero efficiency observed. The generation rate was increased to $1 e^{30} \mathrm{~cm}-3$ before distinctive photovoltaic effects were observed. The result implies that the surface area must be a factor of $10^{6}$ greater than the flat surface area. A. Froimovitch [11] reported NST films 


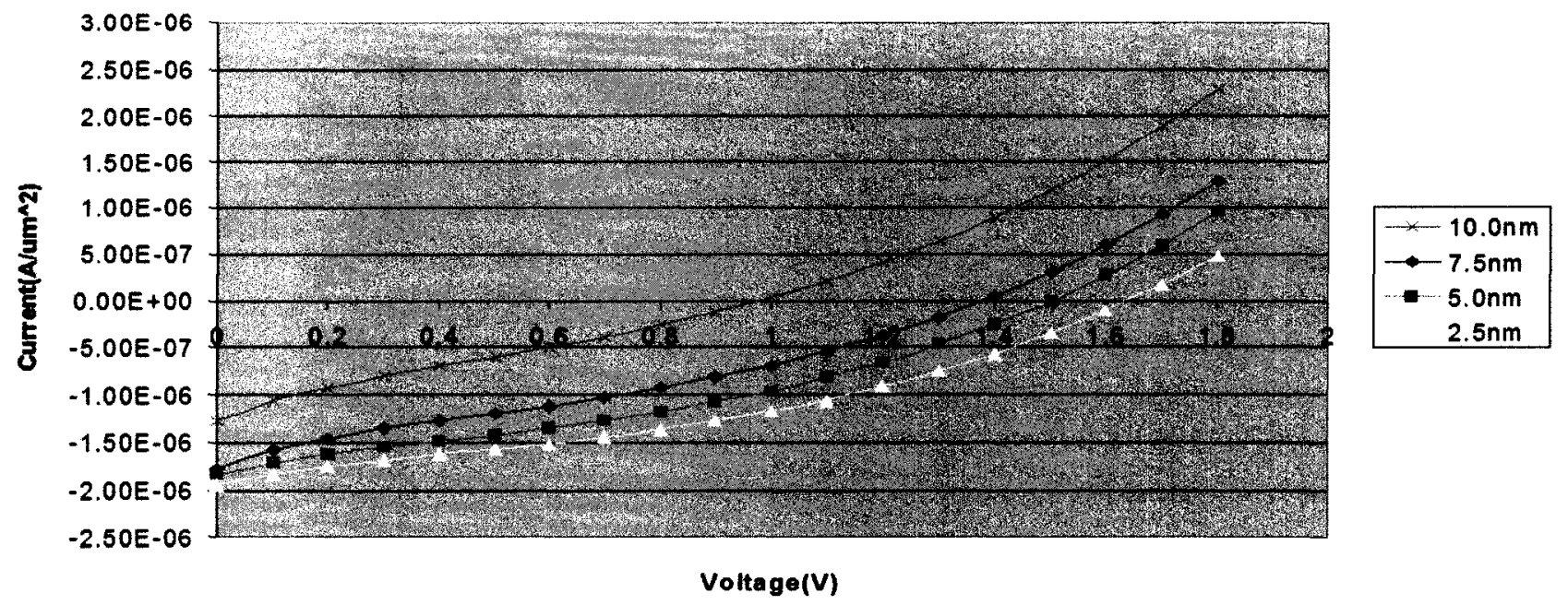

Figure 5.2: IV curve for ITO/Titania/PEDOT device with no structure and varying titania thickness

with density of $535 \frac{\mathrm{m}^{2}}{\mathrm{~g}}$ and surface area increases of factor $10^{3}$. Surface increases of factor $10^{6}$ are theoretically possible with the reported density of NST films but would require a very thick structured layer.

An exponential decrease in efficiency with increasing titania thickness can be seen in figure 5.3. The base layer of titania is therefor kept at a minimum thickness in subsequent simulations to increase efficiency. The decreased efficiency can be explained by the large resistivity of the titania. The longer the path the charge carriers must travel in the titania to reach the ITO, the larger the resistive loss, and the lower the efficiency. The maximum efficiency is $25.60 \%$ with a titania thickness of $2.5 \mathrm{~nm}$. 


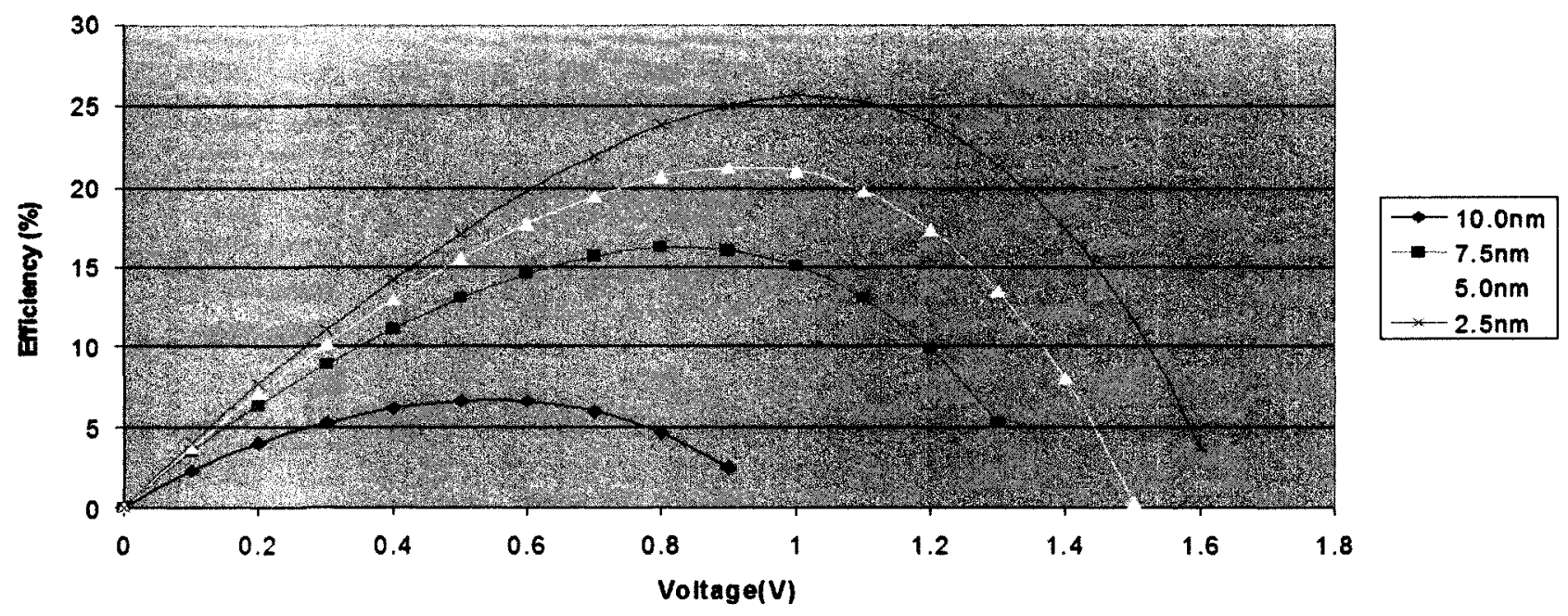

Figure 5.3: Efficiency for ITO/Titania/PEDOT device with no structure and varying titania thickness at $g=1 e^{30} \mathrm{~cm}^{-3}$

\subsection{Two dimensional structuring results}

\subsubsection{Finger structures}

The first structures to be simulated are the very basic finger configuration. The finger configuration consists of a flat structure identical to the baseline results with the titania layer of thickness $10 \mathrm{~nm}$, accompanied with a protrusion of PEDOT into the titania. The titania alternates between thickness $10 \mathrm{~nm}$ and $10 \mathrm{~nm}$ - depth where depth is the depth of the finger structure. The structure resembles a comb with teeth of length equal to finger depth. The depth of the finger is modified and the results recorded. The finger structures are shown in figure 5.4 .

The generation occurs all along the titania/pedot interface, and in the simulations is set to $1 e^{29} \mathrm{~cm}^{-3}$. The total number of charge generation sites in the device is approximately 10 times more than the baseline results, and so the generation is decreases by a factor of 10 accordingly. The depth of the finger is set to $2.5 \mathrm{~nm}, 5.0 \mathrm{~nm}$ and $7.5 \mathrm{~nm}$. This corresponds 


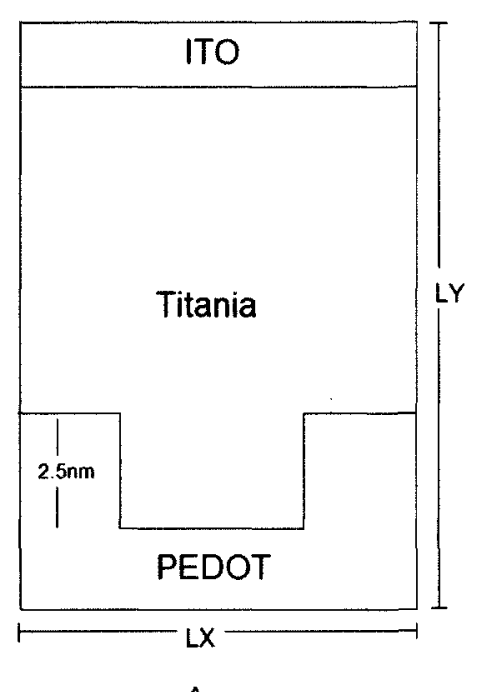

A

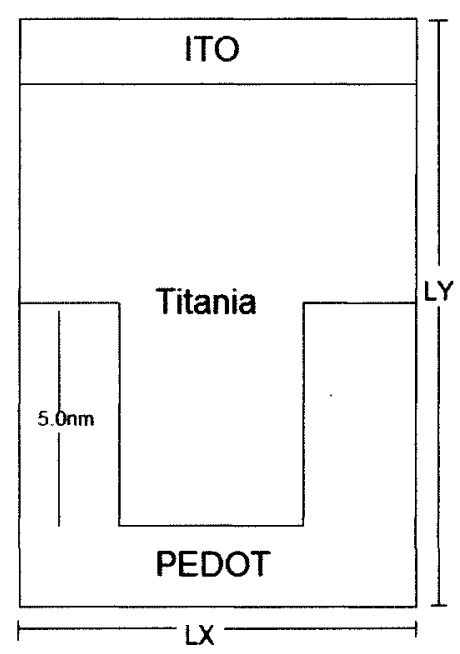

B

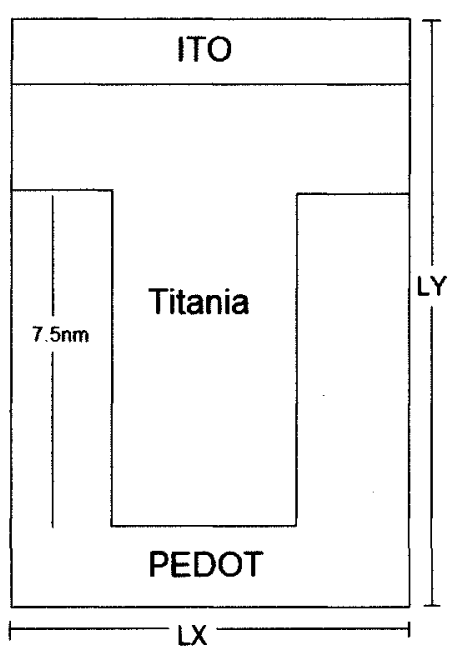

c

Figure 5.4: Pictorial view of finger structures

to a minimum titania depth of $7.5 \mathrm{~nm}, 5.0 \mathrm{~nm}$ and $2.5 \mathrm{~nm}$ respectively. The IV curves are shown in figure 5.5 and the efficiencies for the devices in figure 5.6.

Figure 5.5 shows a dramatic decrease in the IV curve, or increase in power output, as the finger depth increases. The results can be explained by minimum titania thickness. The deeper the finger depth, the lower the minimum titania thickness, and the lower resistive loss across the titania. The finger depth also adds more surface area, and subsequently more charge generation, again leading to higher efficiencies.

The finger structure of depth $7.5 \mathrm{~nm}$ (or minimum titania thickness of $2.5 \mathrm{~nm}$ ) has a maximum efficiency of $23.82 \%$, compared to a baseline efficiency for $2.5 \mathrm{~nm}$ titania thickness of $25.60 \%$. The results are very close, even though the finger structure produces half of its charge more than $5 \mathrm{~nm}$ away from the ITO layer. If the efficiency of the cell depended only on the location of charge generation, one would expect a result for the finger structure similar to that of the $5.0 \mathrm{~nm}$ baseline results, since half of the generation occurs 


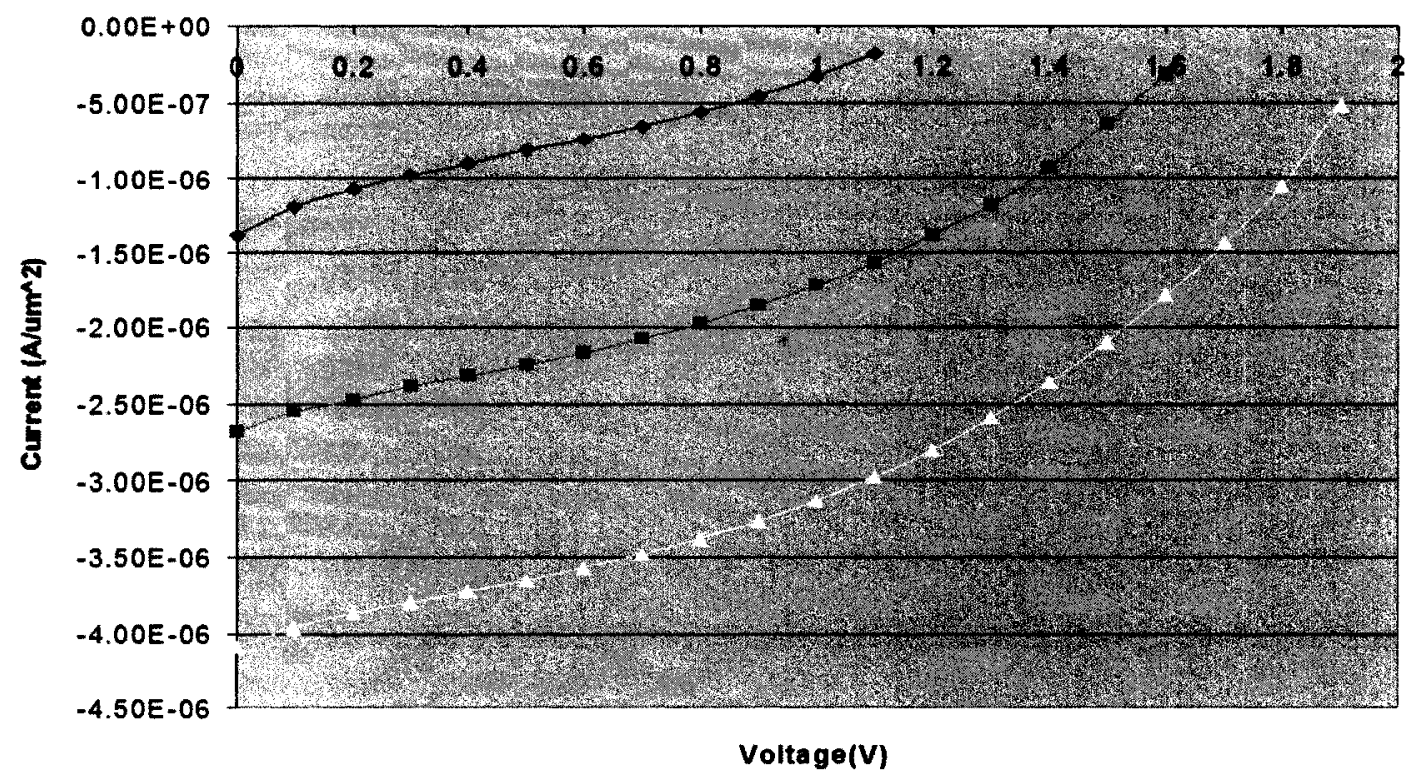
$-2.5 \mathrm{~nm}$ finger
- $5.0 \mathrm{~nm}$ finger $7.5 \mathrm{~nm}$ finger

Figure 5.5: IV curve for ITO/Titania/PEDOT device with finger structure of varying depth

at $2.5 \mathrm{~nm}$ and half at $7.5 \mathrm{~nm}$. The baseline result for efficiency for $5.0 \mathrm{~nm}$ is $21.01 \%$. The higher efficiency result of $23.82 \%$ suggests the minimum titania thickness is a contributing factor to the overall efficiency of the cell.

\subsubsection{Complex structuring}

Four more complex structures were simulated to determine the effects of structuring on cell efficiency. The four structures, along with their generation location matrices are shown in figure 5.8. A simpler 2D pictorial view is shown in figure 5.7.

All of the structure were simulated with generation rate of $1 e^{2} 9 \mathrm{~cm}^{-3}$. The IV curves from the simulations are shown in figure 5.9. The efficiency curves for the structures are shown in figure 5.10. The output graphs for the structures are shown in figures 5.11 through 5.14. The output graphs show the voltage throughout the device, the carrier 


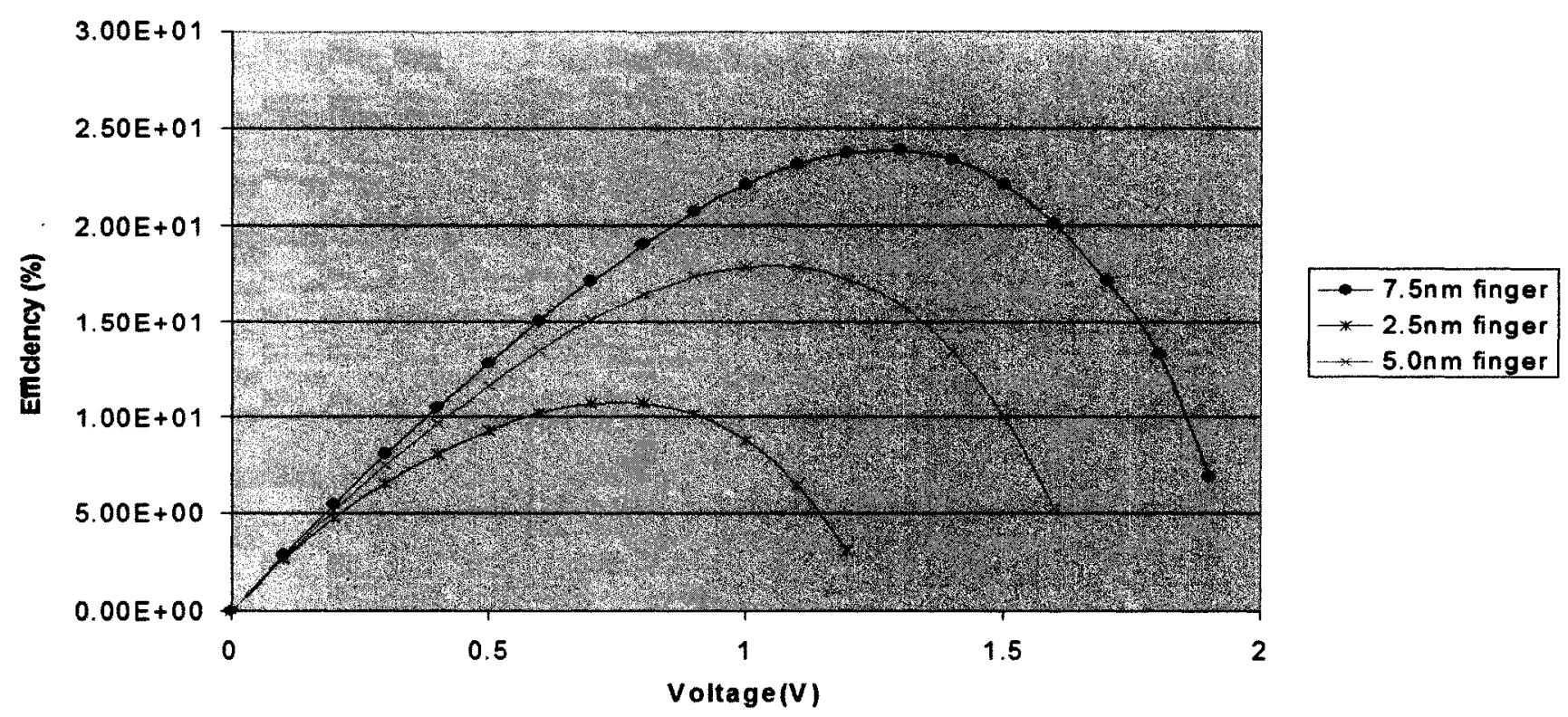

Figure 5.6: Efficiency for ITO/Titania/PEDOT device with finger structure of varying thickness at $g=1 e^{29} \mathrm{~cm}^{-3}$

concentrations, and the log of the carrier concentrations in both surface plots and corresponding contour plot for easier visualization.

The first structure, structure $\mathrm{A}$, consists of a $\mathrm{T}$ shaped titania protrusion on a flat plane of titania of depth $2.5 \mathrm{~nm}$, repeated in the $\mathrm{Y}$ plane. The $\mathrm{T}$ has an advantage over the finger structure in terms of surface area, and the $\mathrm{T}$ shape allows more charge generation closer to the ITO layer. The output graphs show a small depletion region in the flat section of the titania/pedot junction, and an exponentially smaller depletion layer on the bottom side of the $\mathrm{T}$. There is very little effect on the voltage curve, indicating very little electric field within the structure itself. A closer inspection of the voltage plot shows a small electric field at the base of the T pulling charge towards the ITO.

Structure B is again a $\mathrm{T}$ shape, but the top of the $\mathrm{T}$ is pushed farther from the ITO layer. The depletion layer areas are the same as structure A as expected, and again a small 

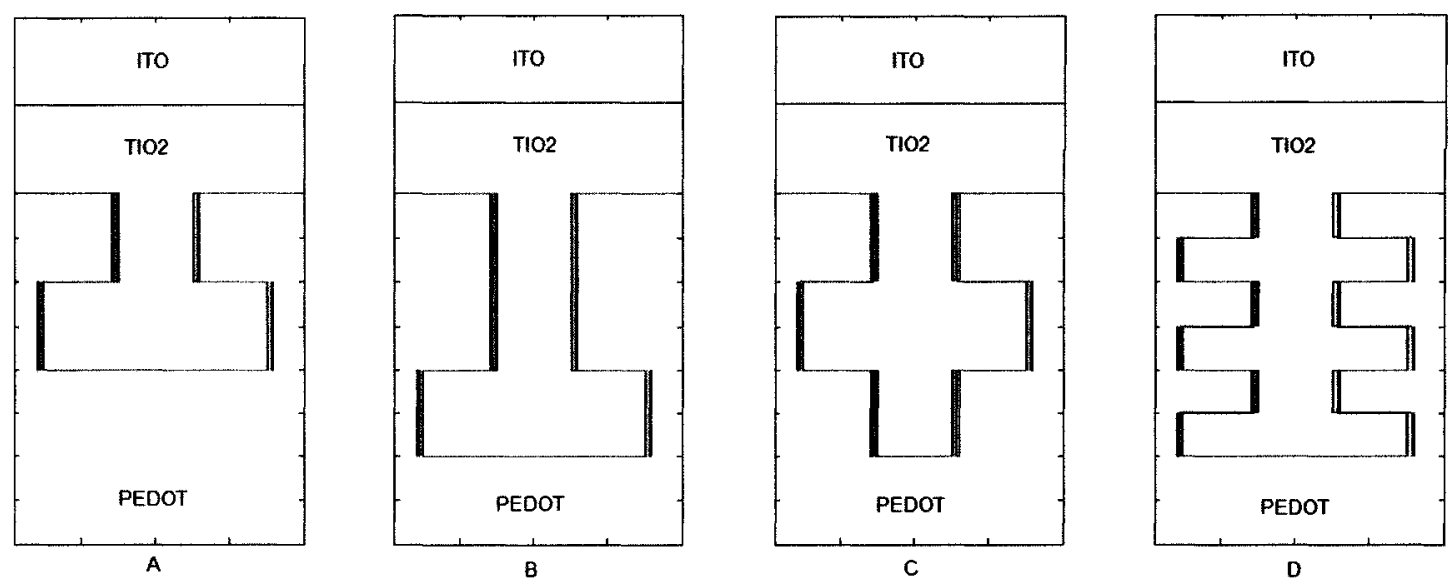

Figure 5.7: Pictorial view of complex structures

voltage gradient produces a small electric field pulling the electrons towards the ITO. The movement of the $\mathrm{T}$ has increases surface area, and consequently raised the power output in relation to structure A. However, the overall efficiency has decreased from $25.76 \%$ to $24.15 \%$. The result indicates some relation between the distance from the ITO of the charge generation and efficiency.

Structure C is a cross shaped structure with identical surface area, and therefore identical charge generation to structure B. The some of the charge however is generated farther away from the ITO layer, and a small decrease in efficiency is expected. The IV curve and efficiency of the structure are almost identical to that of structure $B$, suggesting that the charge generation location and efficiency are not related. Closer inspection of numerical results show that although the maximum efficiency of structure $\mathrm{B}$ and $\mathrm{C}$ is identical, structure $\mathrm{C}$ does have a slightly lower efficiency curve over all. It is thought that the maximum efficiency of structure B is actually slightly higher than reported due to error in interpolation. 
The last structure, structure $\mathrm{D}$, is the most complex structure, and as such has the highest total charge generation. The maximum efficiency is $24.13 \%$, slightly lower than that of structures B and C. Due to the complex shape, more charge is generated at a greater distance from the ITO junction. Based on previous results, a slightly lower efficiency is expected. Again there is a small electric field driving some charge throughout the structure, but the majority of the charge movement is driven by concentration gradients.

Attempting to find an explanation for the seemingly small effect of efficiency on structure size and complexity, the resistance of the structures were plotted. The resistance for structure $\mathrm{D}$ is shown in figure 5.15 and figure 5.16 . The resistance plot shows a very small resistance in the structure itself. Compared to the resistance at the base of the titania, the resistance in the structure is very small and therefore the resistive losses are low.

\subsection{Summary}

The maximum efficiency results are shown in table 5.1. Structure A and the baseline $2.5 \mathrm{~nm}$ cells performed well, giving maximum efficiencies over $25 \%$. In the baseline results, dramatic decrease in efficiency was seen with increasing titania thickness. The finger structures only produced good efficiencies when the titania thickness was minimized. The maximum efficiency achieved with the finger structure was less than the baseline results. The more complex structures produced consistent results all within $1.73 \%$. The maximum efficiencies were seen when the majority of the charge generation was close to the ITO junction. 


\begin{tabular}{|c|c|}
\hline Structure & \\
\hline Maximum efficiency (\%) & 4.0 \\
\hline Flat 2.5nm & 25.61 \\
\hline Flat 5.0nm & 21.01 \\
\hline Flat 7.5nm & 16.23 \\
\hline Flat 10.0nm & 6.58 \\
\hline Finger $2.5 \mathrm{~nm}$ & 10.04 \\
\hline Finger $5.0 \mathrm{~nm}$ & 17.89 \\
\hline Finger 7.5nm & 23.82 \\
\hline Structure A & 25.76 \\
\hline Structure B & 24.15 \\
\hline Structure C & 24.15 \\
\hline Structure D & 24.03 \\
\hline
\end{tabular}

Table 5.1: Maximum efficiency results summary 

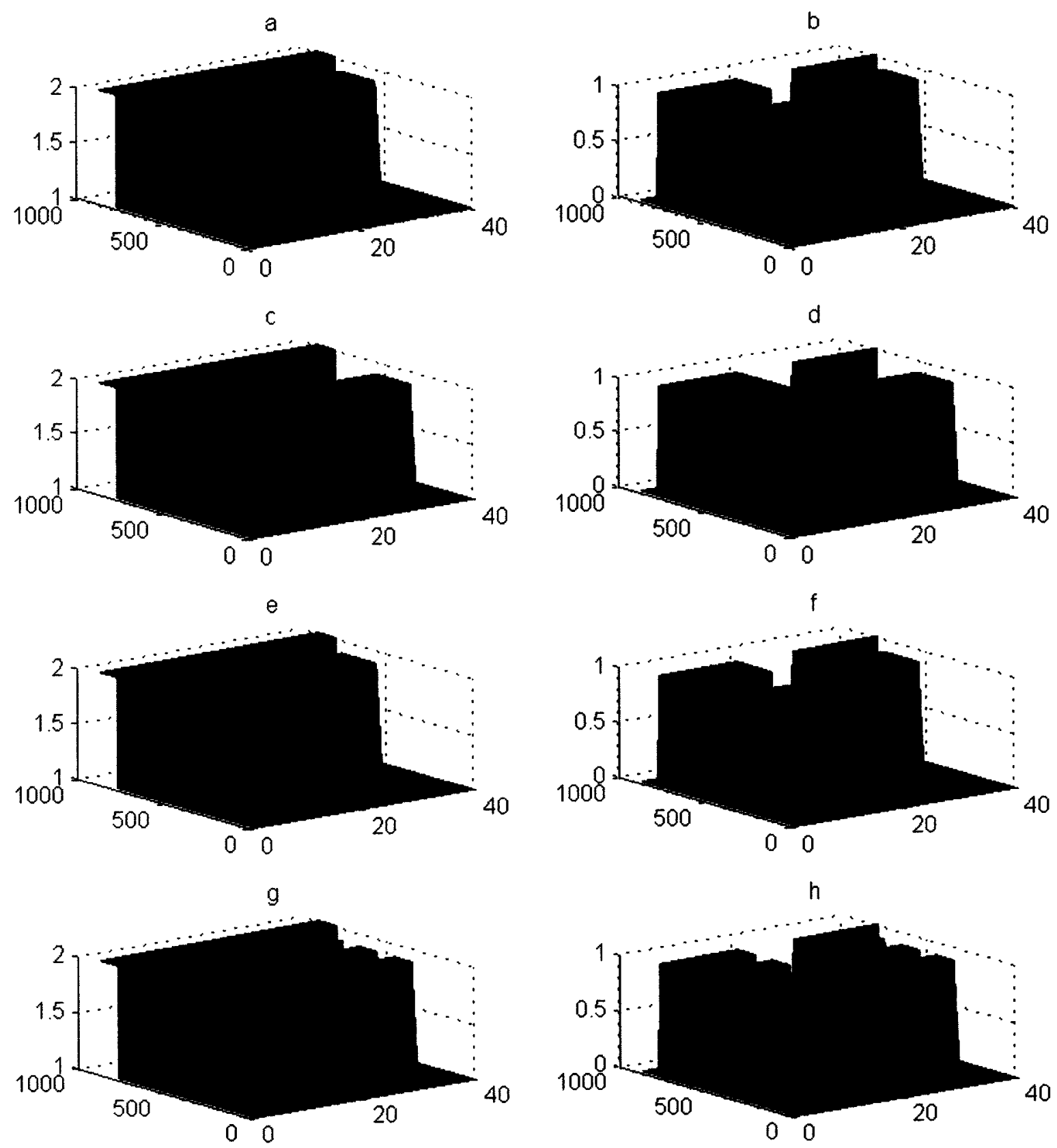

Figure 5.8: Structure shapes (a) (b) (c) (d) and corresponding generation locations (e) (f) $(\mathrm{g})(\mathrm{h})$ 


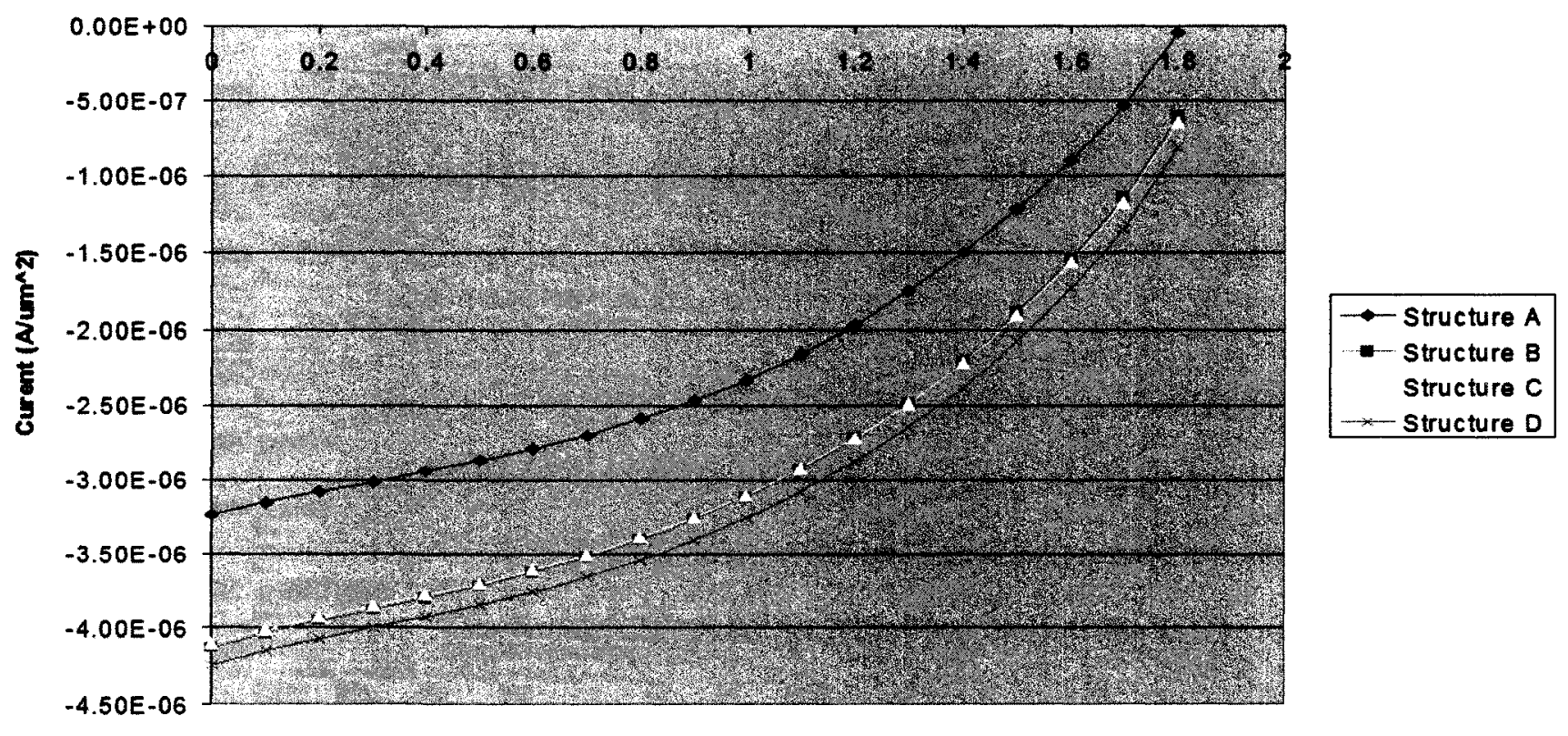

Figure 5.9: IV curve for ITO/Titania/PEDOT device with complex structures (a) through (d)
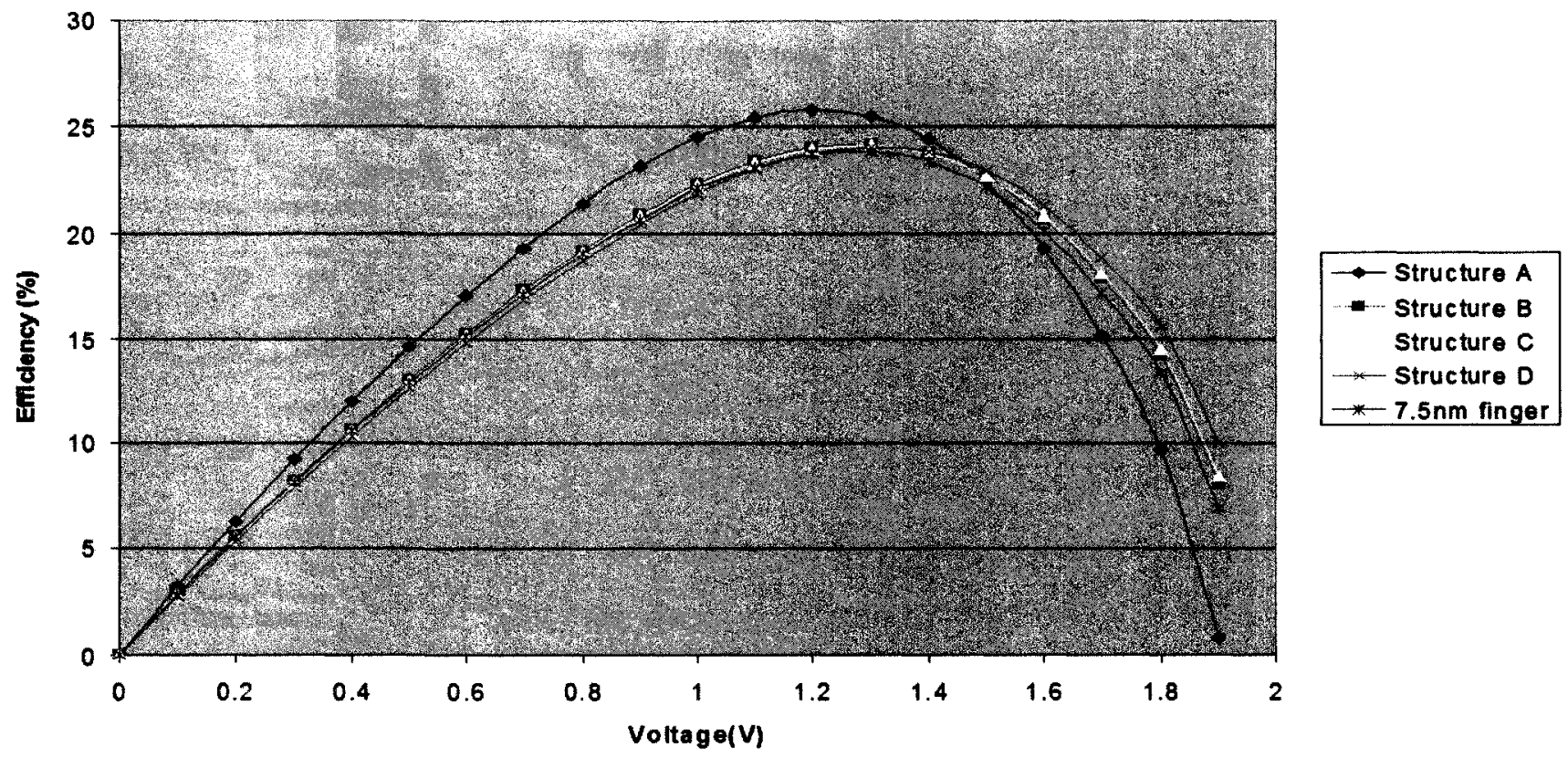

Figure 5.10: Efficiency curves for ITO/Titania/PEDOT device with complex structures (a) through (d) 

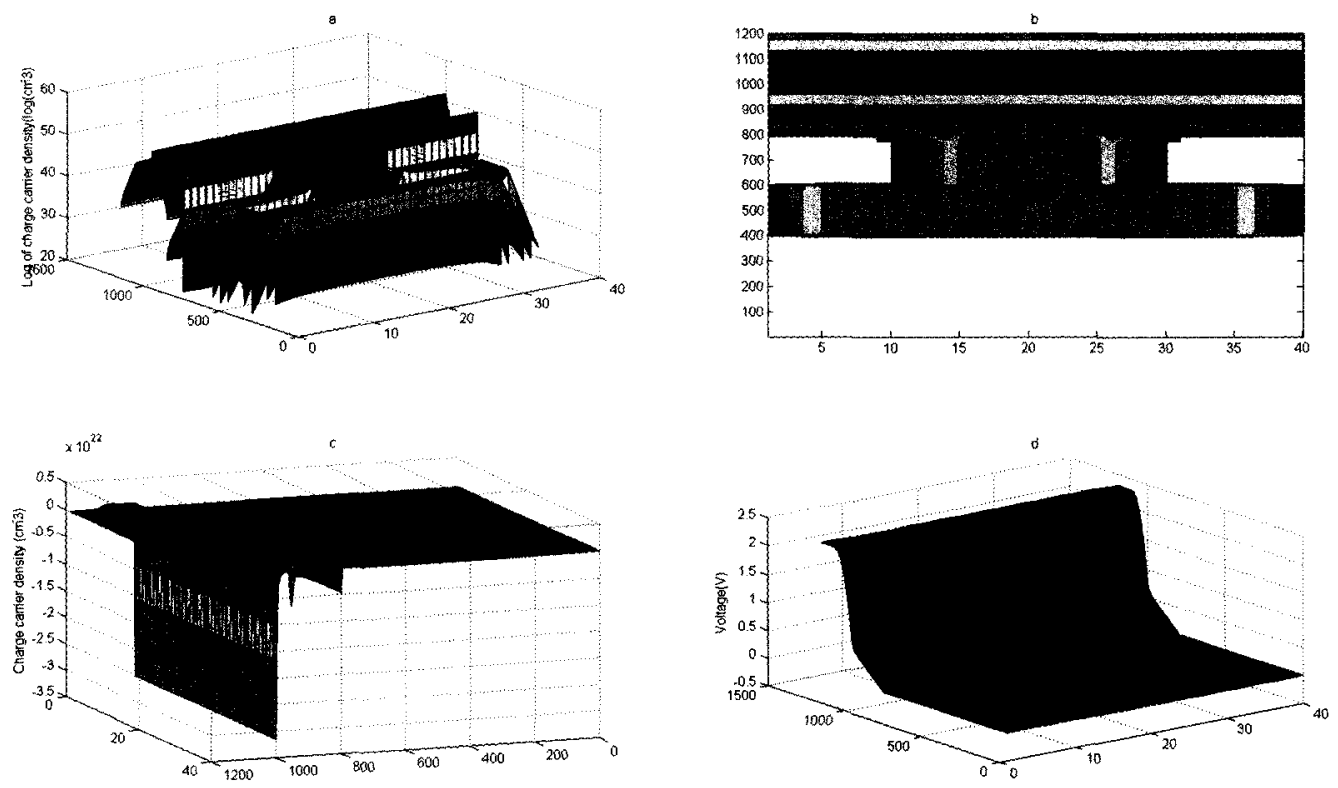

Figure 5.11: Output graphs for structure A (a) Log of carrier concentration surface plot (b) Corresponding contour plot (c) Carrier concentration surface plot (d) Voltage surface plot 

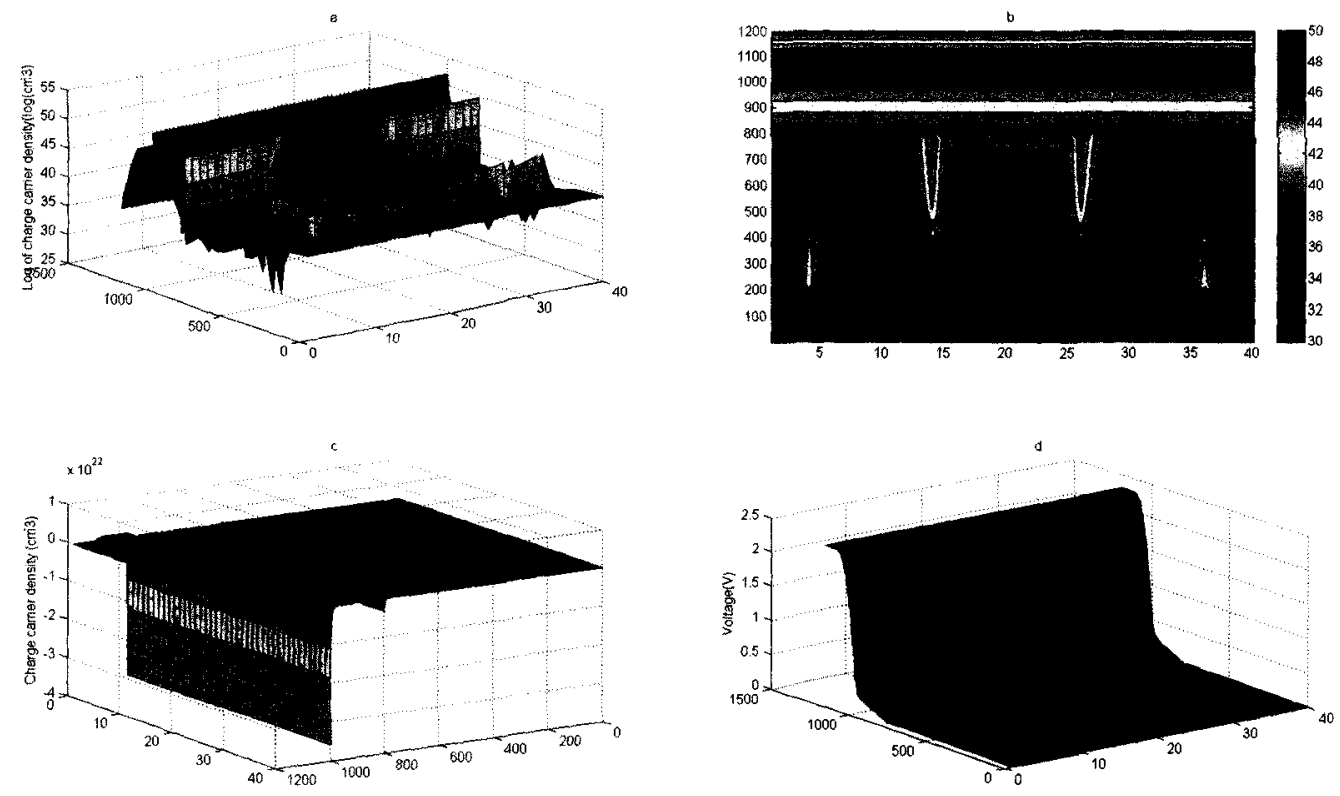

Figure 5.12: Output graphs for structure B (a) Log of carrier concentration surface plot (b) Corresponding contour plot (c) Carrier concentration surface plot (d) Voltage surface plot 

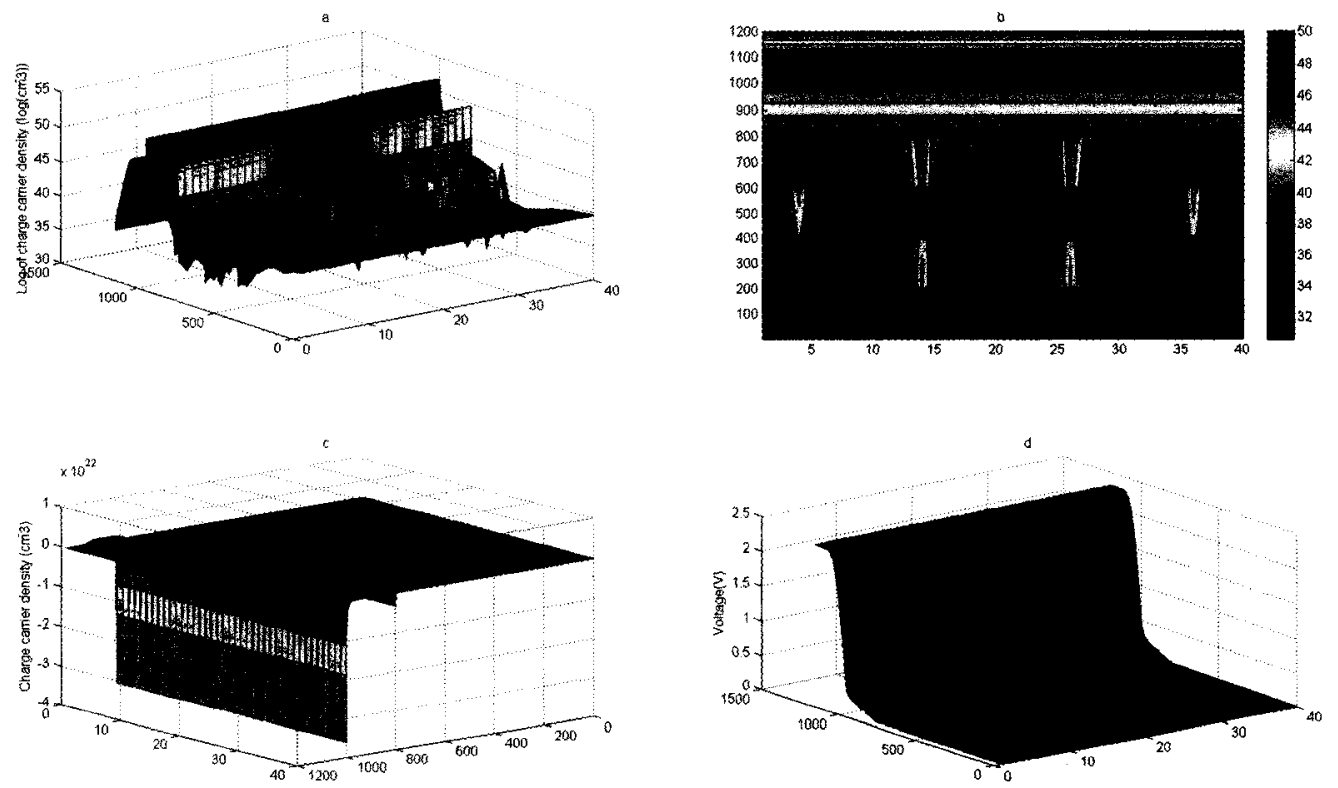

Figure 5.13: Output graphs for structure A (a) Log of carrier concentration surface plot (b) Corresponding contour plot (c) Carrier concentration surface plot (d) Voltage surface plot 

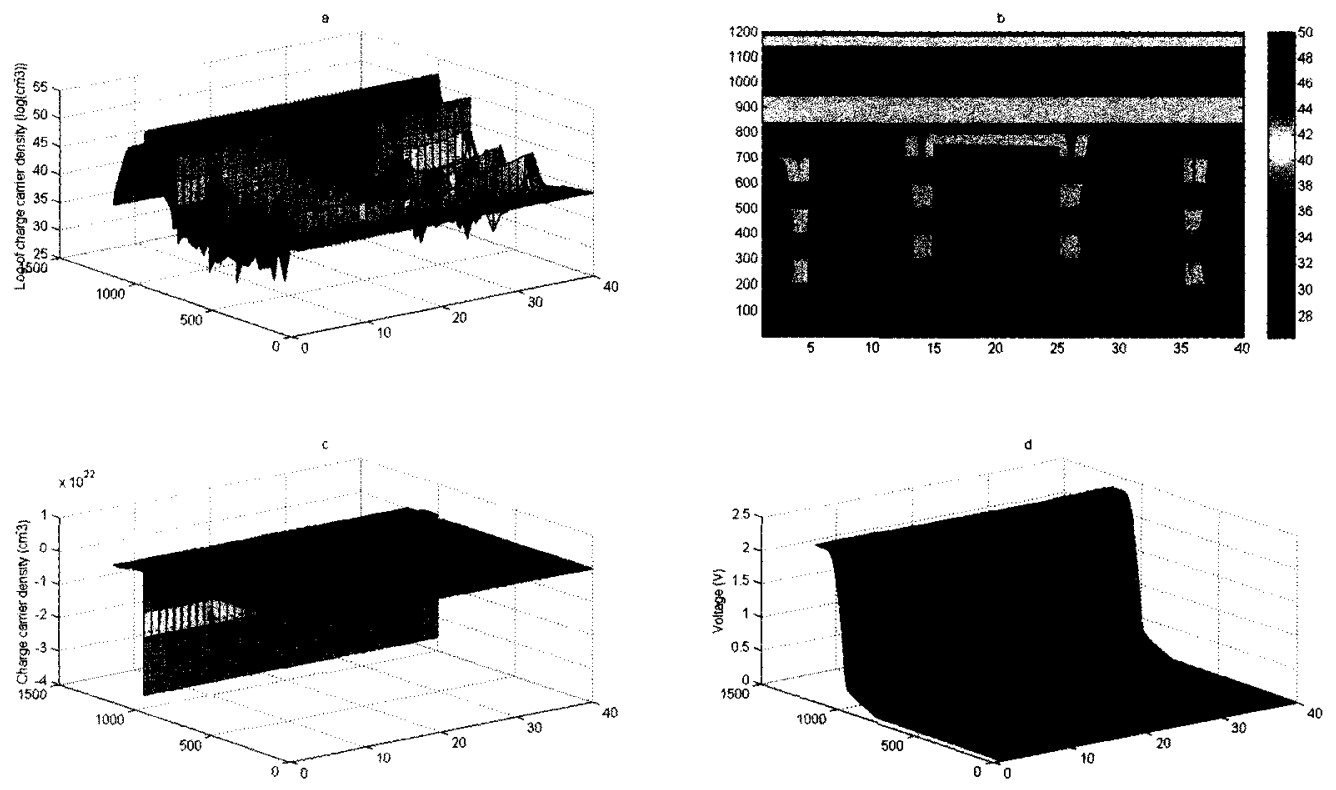

Figure 5.14: Output graphs for structure A (a) Log of carrier concentration surface plot (b) Corresponding contour plot (c) Carrier concentration surface plot (d) Voltage surface plot 


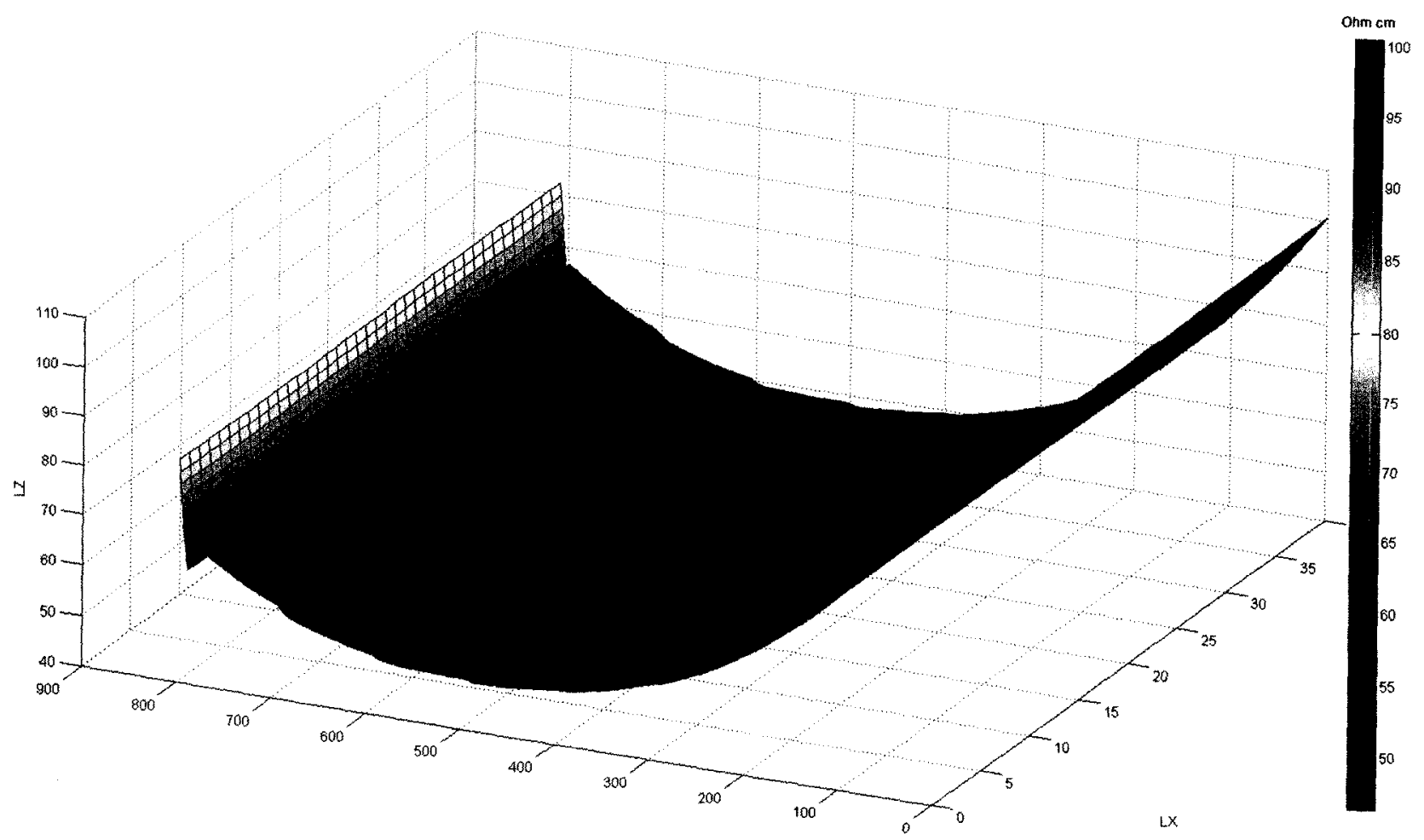

LY

Figure 5.15: Resistance plot for structure D 


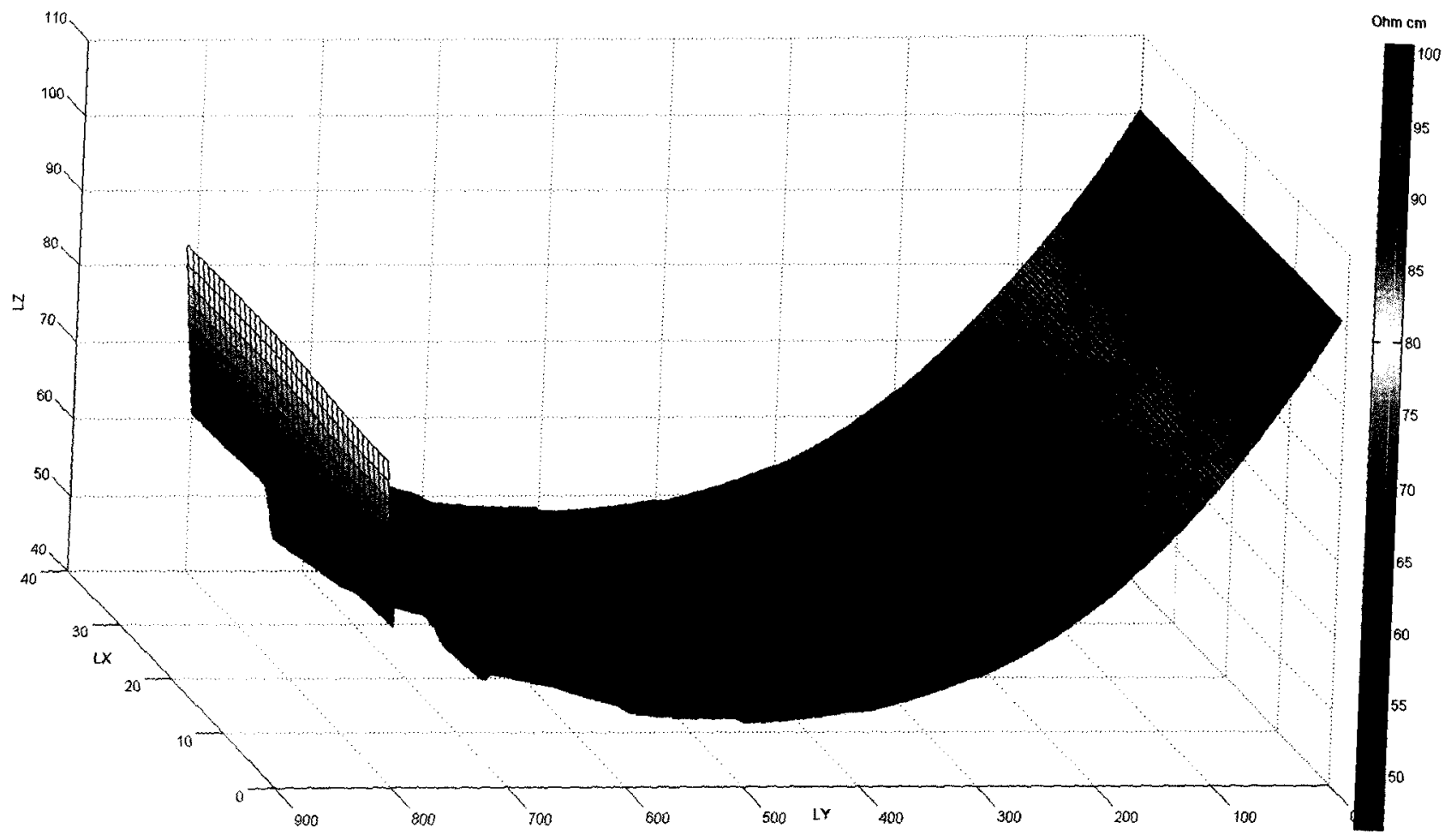

Figure 5.16: Resistance plot for structure D 


\section{Chapter 6}

\section{Conclusions}

This report reviews the possibility of simulating polymer based dye sensitized solar cells by using semiconductor simulation techniques. By using a combination of the semiconductor equations, finite difference methods and the Newton-Raphson algorithm for solving nonlinear problems, a simulator was design for the sole purpose of investigatin the simulation of complex structures inherent in DSSC's. The polymer based DSSC was explored and an equivalent model was determined to allow simulation using semiconducting parameters for each material. The incoming solar radiation at AM1.5 was determined and converted to an equivalent generation rate within the solar devices. Several structures were simulated to determine the effects of structure on photocell efficiency.

The simulator designed is capable of simulating in one, two, and three dimensions. The simulator was tested for accuracy against the commercial simulator MEDICI in both one and two dimensions. The photocells were also simulated in both one and two dimensions. One dimensional simulation involves relatively low computational expense, due to the small size of the matrices involved. In one dimension, the global matrix is of size $3 \mathrm{~N}$, and 
the local matrices are of size $3 \mathrm{X} 9$. In two dimensions, the local matrices increase to $3 \mathrm{X} 15$. The global matrices are still size $3 \mathrm{~N}$, but the number of elements is now $N=n_{x} n_{y}$ instead of simply $N=n_{x}$. In three dimensions, the number of elements becomes $N=n_{x} n_{y} n_{z}$ and the local matrices are of size $3 \times 21$. As long as the total $\mathrm{N}$ is kept at a reasonable size, simulations can take place in a reasonable time period. However, the structuring in DSSC's involves using very small element sizes which in turn requires a large N.

The simulation results from varying structures show efficiencies in the range of $25 \%$. These efficiencies assume a incoming photon to current efficiency(IPCE) of $100 \%$ in the 450 to $750 \mathrm{~nm}$ range, which is extremely unlikely in any circumstance. A more realistic IPCE is in the range of $30-40 \%$, which means a total efficiency of $7.5-10 \%$. Compared to measured experimental values in the range of $4 \%$, the values are very high. The generation rate in the simulations is increased substantially over AM1.5 to compensate for the simpler structures and lower surface area ratios. It is believed based on the results presented here that efficiencies in the range of it least $10 \%$ could be achieved if surface area ratios of the titania structure approached the $10^{6}$ value.

The simulated structures are very simple compared to actual nano structured films used for experimental work. By simulating the structures, the intention was to determine some basic features of the titania film that contribute to higher efficiency. The results show that the main factor contributing to the cell efficiency is the base thickness of the titania structure, or the minimum titania thickness in the device. Minimizing the base layer maximizes the cell efficiency. The layer was reduced to a minimum of $2.5 \mathrm{~nm}$ in the simulations, and was not reduced further due to the size of the elements. It is hypothesized 
that this layer can be reduced further creating greater efficiency. The location of the charge generation also seems to affect the efficiency, but to a much smaller degree. Resistivity plots show the charge generation causes a very low resistivity in the titania structure leading to minimal losses. By maintaining a relatively compact structure, a maximum surface area can be produced with minimum distance for generated charge to travel. It is believed that the large resistivity losses in the base titania layer are due to the artificially high generation needed to produce results on simplified simulations.

After closer inspection of the results and comparisons to the original band diagrams of the device, it is believed that an artificial barrier was created at the PEDOT/titania interface. In actual devices, the dye provides an energy barrier assuring charge separation and stopping electrons from crossing back over the interface and recombining. The simulation with PVSIM artificially separates the charge to model the function of the dye, and the finite element size coupled with the small mobility values in PEDOT creates a barrier preventing recombination.

Although the simulations produced some valuable information on the structuring of titania films, at this time it is not feasible to accurately simulate DSSC's using numerical techniques. First and foremost, the computational time needed to simulate a realistic structured film is large, even if more advanced sparse matrix techniques are implemented. As the grid must encompass all three materials of the device, and the element size must be small, the number of elements needed is very large. The assumptions made in section 4.1 for material parameters were designed to add simplicity to the simulations. The current research concerning the charge movement in PEDOT, ITO and titania is very limited 
and results vary tremendously. The structuring of the titania itself produces problems as well, as charge transport in NST titania may not have the same mechanisms as charge transport in bulk titania.

The results of this work show very little dependence of the efficiency of the cell on the geometry of the titania structure. It is suggested that for future research, the structured film be treated as a bulk substrate with material parameters deduced from experimental work. This would simplify simulation grids and allow for large simulations. An electromagnetic analysis of structure with incoming light could be very informative, and provide information on how various structures harvest light and produce higher IPCE's. The coupling of solar concentrators with DSSC's could produce dramatic increases in efficiencies by increasing charge generation without increasing surface area. 
Appendix A - 3D Equations

$\mathrm{X}$ vector

$$
\left[\begin{array}{c}
\hat{V}_{x-1} \\
\hat{V}_{x+1} \\
\hat{V}_{y-1} \\
\hat{V}_{y+1} \\
\hat{V}_{z-1} \\
\hat{V}_{z+1} \\
\hat{V} \\
n_{x-1} \\
n_{x+1} \\
n_{y-1} \\
n_{y+1} \\
n_{z-1} \\
n_{z+1} \\
n \\
p_{x-1} \\
p_{x+1} \\
p_{y-1} \\
p_{y+1} \\
p_{z-1} \\
p_{z+1} \\
p
\end{array}\right]
$$

\section{$B$ vector}

$$
\left[\begin{array}{c}
s_{2}\left(N_{A}-N_{D}\right) \\
0 \\
0
\end{array}\right]
$$

where 


$$
\hat{V}=s_{1} V \varepsilon_{r}
$$

Non-linear vector $\mathrm{f}(\mathrm{x})$ row 2 , row 1 zeros, row 3 same but negative and with $\mathrm{p}$.

$$
\frac{1}{s_{1} h^{2}}\left[\begin{array}{l}
n_{x-1} \mu N_{x-1} \hat{V}_{x-1} / \varepsilon_{x-1}+n_{x+1} \mu N_{x+1} \hat{V}_{x+1} / \varepsilon_{x+1}+n_{y-1} \mu N_{y-1} \hat{V}_{y-1} / \varepsilon_{y-1} \\
+n_{y+1} \mu N_{y+1} \hat{V}_{y+1} / \varepsilon_{y+1}+n_{z-1} \mu N_{z-1} \hat{V}_{z-1} / \varepsilon_{z-1}+n_{z+1} \mu N_{z+1} \hat{V}_{z+1} / \varepsilon_{z+1}-6 n \mu N \hat{V} / \varepsilon
\end{array}\right]
$$

A matrix row 1

$$
\begin{aligned}
& \begin{array}{lllllll}
\frac{s_{2} \varepsilon_{o}}{s_{1} q h^{2}} & \frac{s_{2} \varepsilon_{o}}{s_{1} q h^{2}} & \frac{s_{2} \varepsilon_{o}}{s_{1} q h^{2}} & \frac{s_{2} \varepsilon_{o}}{s_{1} q h^{2}} & \frac{s_{2} \varepsilon_{o}}{s_{1} q h^{2}} & \frac{s_{2} \varepsilon_{o}}{s_{1} q h^{2}} & -6 \frac{s_{2} \varepsilon_{o}}{s_{1} q h^{2}}
\end{array} \\
& \begin{array}{llllllllllllll}
0 & 0 & 0 & 0 & 0 & 0 & -s_{2} & 0 & 0 & 0 & 0 & 0 & 0 & s_{2}
\end{array}
\end{aligned}
$$

A matrix row 2

$$
\begin{array}{ccccccc}
0 & 0 & 0 & 0 & 0 & 0 & 0 \\
\frac{D N_{x-1}}{h^{2}} & \frac{D N_{x+1}}{h^{2}} & \frac{D N_{y-1}}{h^{2}} & \frac{D N_{y+1}}{h^{2}} & \frac{D N_{z-1}}{h^{2}} & \frac{D N_{z+1}}{h^{2}} & -6 \frac{D N}{h^{2}} \\
0 & 0 & 0 & 0 & 0 & 0 & 0
\end{array}
$$

A matrix row 3

$$
\begin{array}{cccccccccccccc}
0 & 0 & 0 & 0 & 0 & 0 & 0 & 0 & 0 & 0 & 0 & 0 & 0 & 0 \\
\frac{D P_{x-1}}{h^{2}} & \frac{D P_{x+1}}{h^{2}} & \frac{D P_{y-1}}{h^{2}} & \frac{D P_{y+1}}{h^{2}} & \frac{D P_{z-1}}{h^{2}} & \frac{D P_{z+1}}{h^{2}} & -6 \frac{D P}{h^{2}} & & & & & & &
\end{array}
$$

Jacobian terms

$$
\left[\begin{array}{lllllll}
\frac{n_{x-1} \mu N_{x-1}}{s_{1} h^{2} \varepsilon_{x-1}} & \frac{n_{x+1} \mu N_{x+1}}{s_{1} h^{2} \varepsilon_{x+1}} & \frac{n_{y-1} \mu N_{y-1}}{s_{1} h^{2} \varepsilon_{y-1}} & \frac{n_{y+1} \mu N_{y+1}}{s_{1} h^{2} \varepsilon_{y+1}} & \frac{n_{z-1} \mu N_{z-1}}{s_{1} h^{2} \varepsilon_{z-1}} & \frac{n_{z+1} \mu N_{z+1}}{s_{1} h^{2} \varepsilon_{z+1}} & -6 \frac{n \mu N}{s_{1} h^{2} \varepsilon}
\end{array}\right]
$$




\section{References}

[1] M. Gratzel, "Photoelectrochemical cells," Nature, vol. 414, 2001.

[2] E. Lorenzo, Solar Electricity: Engineering of Photovoltaic Systems. Progensa, 1994.

[3] N. R. energy lab, http://www.nrel.gov/ncpv/new_in_cpv.html.

[4] Spectrolab, http://www.energy.gov/news/4503.htm.

[5] S. M. Sze, Physics of Semiconductor Devices. New York: John Wiley and Sons, 1981.

[6] M. Gratzel, "Conversion of sunlight to electric power by nanocrystalline dye sensitized solar cells," Journal of Photochemistry and Photobiology A: Chemistry, vol. 164, pp. 3-14, 2004.

[7] A. C. Arango, L. R. Johnson, V. N. Bliznyuk, Z. Schlesinger, S. A. Carter, and H. H. Horhold, "Efficient titanium oxide/conjugated polymer photovoltaics for solar energy conversion," Advanced Materials, vol. 12, no. 22, pp. 1689-1692, 2000.

[8] P. Ravirajan, S. A. Haque, J. R. Durrant, D. Poplavskyy, D. D. C. Bradley, , and J. Nelson, "Hybrid nanocrystalline $\mathrm{TiO} 2$ solar cells with a fluorene- thiophene copolymer as a sensitizer and hole conductor," J. Appl. Phys., vol. 3, no. 95, pp. 1473-1480, 2004.

[9] L. Ding, M. Jonforsen, L. Roman, M. Andersson, and O. Inganas, "Photovoltaic cells with a conjugated polyelectrolyte," Synthetic Metals, vol. 110, pp. 133-140, 2000.

[10] P. Ravirajan, A. Green, S. A. Haque, J. R. Durrant, D. D. C. Bradley, and J. Nelson, "Efficient hybrid polymer/TiO2 solar cells using a multilayer structure," Organic Photovoltaics, vol. 5520, pp. 777-786, 2004.

[11] A. Froimovitch, "Nanoporous and Nanostructured Aluminium and Titanium Oxide Thin Films," Carleton University, September 2006.

[12] A. V. D. Ziel, Solid State Physical Electronics. New York: Prentice Hall, 1957.

[13] F. B. Hildebrand, Finite-Difference Equations and Simulations. New Jersey: Prentice-Hall, 1968. 
[14] W. F. Ames, Numerical Method for Partial Differential Equations. New York: Academic Press, 1977.

[15] J. Vlach, Computer Methods for Circuit Analysis and Design. Boston: Kluwer Academic, 1994.

[16] P. M. Morse and H. Feshbach, Methods of Theoretical Physics. New York: McGrawHill, 1953.

[17] Synopsys, "Taurus Medici Documentation Manual," 2000.

[18] C. Demetry and S. Xinlan, "Grain size-dependent electrical properties of rutile (TiO2)," Solid State Ionics, vol. 118, no. 3-4, pp. 271-279, March 1999.

[19] A. P. Huang, P. Chu, L. Wang, W. Y. Cheung, J. B. Xu, and S. P. Wong, "Fabrication of rutile $\mathrm{TiO} 2$ thin films by low-temperature, bias-assisted cathodic arc deposition and their dielectric properties," Solid State Ionics, vol. 21, no. 4, pp. 844-850, April 2006.

[20] S. Tsunekawa, K. Baba, and T. Fukuda, "Dielectric properties of semiconducting rutile crystals," Materials Transactions, vol. 34, no. 7, pp. 630-633, July 1993.

[21] T. Bak, J. Nowotny, M. Rekas, and C. Sorrell, "Defect chemistry and semiconducting properties of titanium dioxide: III. Mobility of electronic charge carriers," Journal of Physics and Chemistry of Solids, vol. 64, pp. 1069-1087, DEC 2003.

[22] T. Bak and J. Nowotny, "Defect chemistry and semiconducting properties of titanium dioxide: I. Intrinsic electronic equilibrium," Journal of Physics and Chemistry of Solids, vol. 64, no. 64, pp. 1043-1056, July 2003.

[23] A. Dey, S. De, A. De, and S. De, "Giant dielectric constant in titania nanoparticles embedded in conducting polymer matrix," Journal of Nanoscience and Nanotechnology, vol. 6, no. 5, pp. 1427-1436, MAY 2006.

[24] X.-s. Ye, Z.-g. Xiao, D.-s. Lin, S.-y. Huang, and Y.-h. Man, "Experimental investigation on the dielectric behavior of nanostructured rutile-phase titania," Materials Science and Engineering B: Solid-State Materials for Advanced Technology, vol. 74, no. 1-3, pp. 133-136, MAY 2000.

[25] Z. Wing, B. Wang, and J. Halloran, "Permittivity of porous titanate dielectrics," Journal of the American Ceramic Society, vol. 89, no. 12, pp. 3696-3700, DEC 2006.

[26] Z. Haider and M. Shah, "Study of electrical characteristics of some solid dielectric materials," Journal of Nanoscience and Nanotechnology, vol. 27, no. 1-4, pp. 196-200, MAY 1975. 
[27] F. Matino, L. Persano, V. Arima, D. Pisignano, R. I. R. Blyth, R. Cingolani, and R. Rinaldi, "Electronic structure of indium-tin-oxide films fabricated by reactive electron-beam deposition," Physical Review, no. 4, pp. 609-709, April 2005.

[28] M. Batzill and U. Diebold, "The surface and materials science of tin oxide," Progress in Surface Science, no. 79, pp. 47-154, 2005.

[29] T. Nagatomo, Y. Maruta, and A. Omoto, "Electrical and optical properties of vacuumevaporated indium-tin oxide films with high electron mobility," Thin Solid Films, no. 192 , pp. 17-25, 1990.

[30] D. Wakizaka, T. Fushimi, H. Ohkita, and S. Ito, "Hole transport in conducting ultrathin films of PEDOT/PSS prepared by layer-by-layer deposition technique," Polymer, no. 45, p. 85618565, 2004.

[31] H. Mu and D. Klotzkin, "Measurement of Electron Mobility in Alq3 From Optical Modulation Measurements in Multilayer Organic Light-Emitting Diodes," Journal of display technology, vol. 2, no. 4, pp. 341-347, December 2006.

[32] P. Ravirajan, S. Haque, J. Durrant, D. Bradled, and J. Nelson, "The effect of polymer optoelectronic properties on the performance of multilayer hybrid polymer/TiO2 solar cells," Advanced Functional Materials, no. 72, pp. 1-6, August 2005.

[33] L. Ding, M. Jonforsen, L. Roman, M. Andersson, and O. Ingan, "Photovoltaic cells with a conjugated polyelectrolyte," Synthetic Metals, no. 110, pp. 133-140, 2000.

[34] Y. Tachibana, K. Hara, K. Sayama, and H. Arakawa, "Quantitative Analysis of LightHarvesting Efficiency and Electron-Transfer Yield in Ruthenium-Dye-Sensitized Nanocrystalline TiO2 Solar Cells," Cheamical Materials, no. 14, pp. 2527-2535, 2002.

[35] A. Fillinger and B. A. Parkinson, Journal of The Electrochemical Society, no. 146, pp. $4559-4564$.

[36] P. Persson and M. J. Lundqvist, "Calculated Structural and Electronic Interactions of the Ruthenium Dye N3 with a Titanium Dioxide Nanocrystal," Journal of Physics and Chemistry, no. 109, pp. 11918-11924, 2005.

[37] Y. Lu, M. T. Spitler, and B. A. Parkinson, "Photochronocoulometric Measurement of the Coverage of Surface-Bound Dyes on Titanium Dioxide Crystal Surfaces," Journal of Physics and Chemistry, vol. 12, no. 110, pp. 25 273-25 278, 2006. 